\title{
Associations between Sleep, Infant Feeding Methods, Brain Development and Behavior: A Multimodal Approach to Assess Plasticity in the Brain
}

Christopher Edward Bauer

Follow this and additional works at: https://researchrepository.wvu.edu/etd

\section{Recommended Citation}

Bauer, Christopher Edward, "Associations between Sleep, Infant Feeding Methods, Brain Development and Behavior: A Multimodal Approach to Assess Plasticity in the Brain" (2017). Graduate Theses,

Dissertations, and Problem Reports. 5164.

https://researchrepository.wvu.edu/etd/5164

This Dissertation is protected by copyright and/or related rights. It has been brought to you by the The Research Repository @ WVU with permission from the rights-holder(s). You are free to use this Dissertation in any way that is permitted by the copyright and related rights legislation that applies to your use. For other uses you must obtain permission from the rights-holder(s) directly, unless additional rights are indicated by a Creative Commons license in the record and/ or on the work itself. This Dissertation has been accepted for inclusion in WVU Graduate Theses, Dissertations, and Problem Reports collection by an authorized administrator of The Research Repository @ WVU.

For more information, please contact researchrepository@mail.wvu.edu. 
Associations between Sleep, Infant Feeding Methods, Brain Development and Behavior: A Multimodal Approach to Assess Plasticity in the Brain

Christopher Edward Bauer, BA Honors Psychology and Biology

Dissertation Submitted to the

Blanchette Rockefeller Neurosciences Institute at West Virginia University

in partial fulfillment of the requirement for the degree of Doctor of Philosophy in Neuroscience

\author{
Mary Beth Mandich. Ph.D., Chair \\ Hawley Montgomery-Downs, Ph.D. \\ Julie Brefczynski-Lewis, Ph.D. \\ James W. Lewis, Ph.D. \\ Gary Marano, M.D. \\ Marc Haut, Ph.D.
}

Morgantown, WV

2017

Keywords: Sleep-Disordered Breathing, Breastfeeding, Rapid Eye Movement (REM), White Matter, Brain Development, Magnetic Resonance Imaging (MRI), Polysomnography (PSG) 


\begin{abstract}
Associations between Sleep, Infant Feeding Methods, Brain Development and Behavior: A Multimodal Approach to Assess Plasticity in the Brain

\section{Christopher Edward Bauer}

Purpose: Sleep-disordered breathing (SDB) is a spectrum disorder that is estimated to effect a significant proportion of the pediatric population (1-3\% in USA). SDB is able to disrupt and fragment sleep through frequent arousals and intermittent hypoxia. In addition, the long term effects of SDB in pediatrics have been well-documented; decreases in intelligence quotient (IQ), executive function, school performance, and alertness have all been observed. Although surgical treatments can be quite effective, there are no widely accepted prophylactic measures to prevent SDB development. Recently, breastfeeding duration in infancy has been demonstrated to be correlated with reduced SDB (lower AHI, RAI, and higher SpO2), as well as increases in IQ, executive function, and school performance (independent of SDB). The overarching goal of this dissertation was to examine the potential effects of both breastfeeding duration and SDB severity on the neurological underpinnings associated with observed behavioral and cognitive deficits; namely, correlations with white matter structural volume and fractional anisotropy (FA) scores through diffusion tensor imaging (DTI). Here, I proposed a unique developmental hypothesis where breastfeeding may ultimately reduce SDB, enabling the preservation of rapid eye movement (REM) sleep, and leading to healthier neurological white matter development.

Method: Twenty-four children with SDB and 19 healthy controls were imaged using MR techniques. White matter volume was measured using the central 13 millimeters of the corpus callosum (CC). DTI of major white matter tracts was also conducted. The SDB group received neurocognitive testing to assess cognitive performance; the control group was assessed using real-world academic report cards. Finally, REM sleep was quantified in infants using overnight polysomnography (PSG), with SDB metrics and infant feeding method also measured.

Results: There was no correlation between infant feeding methods and CC volume in either group, nor a significant differences between CC volumes in children with SDB versus those without. However, increased breastfeeding duration was correlated with increased left superior longitudinal fasciculus (LSLF) and left angular bundle (LAB) FA scores in healthy controls. In 8-9 month old infants, increased breastfeeding duration was also correlated with a reduced proportion of REM sleep (\%TST), and children with exclusive breastfeeding had reduced SDB in infancy compared to children with any formula feeding. Finally, exclusively formula-fed infants were diagnosed with "primary snoring" more often than those with any amount of breastfeeding.

Conclusions: The findings in this dissertation revealed associations between breastfeeding, SDB, REM sleep, and white matter integrity in the brain. These results support the hypothesis that certain cognitive effects associated with SDB and infant feeding methods may have common underlying anatomical brain changes that subserve these observed phenomena. 
Associations between Sleep, Infant Feeding Methods, Brain Development and Behavior: A Multimodal Approach to Assess Plasticity in the Brain

\section{Christopher Edward Bauer}

Purpose: Sleep-disordered breathing (SDB) may affect a large number of children (1-3\% in USA). SDB is able to disrupt and change sleep through frequent arousals and depriving the blood and tissues of oxygen. The long term effects of SDB in children have been well-documented; decreases in intelligence quotient (IQ), executive function, school performance, and alertness have all been observed. Although surgical treatments can be quite effective, there are no widely accepted non-invasive preventative measures for SDB. Recently, breastfeeding duration in infancy has been shown to be correlated with decreased SDB (lower AHI, RAI, and higher SpO2), as well as increases in IQ, executive function, and school performance (independent of SDB). The major goal of this dissertation was to examine the potential effects of both breastfeeding duration and SDB severity on the biological basis of observed behavioral and cognitive problems. Here, I proposed a unique developmental hypothesis through which preserved rapid eye movement (REM) sleep is essential, and that through breastfeeding SDB might be reduced, leading to healthier sleep and neurological development.

Method: Twenty-four children with SDB and 19 healthy controls were imaged using magnetic resonance techniques. White matter volume was measured using the central 13 millimeters of the corpus callosum (CC). Diffusion tensor imaging of white matter tracts was also conducted. The SDB group received cognitive testing to assess performance; the control group was measured using real-world academic report cards. Finally, REM sleep was measured in infants using overnight polysomnography (PSG), with SDB metrics and infant feeding method also measured.

Results: There was no correlation between infant feeding methods and CC volume in either group, nor any differences between groups. However, children with increased breastfeeding duration had increased white matter connectivity in the left side of the brain in healthy controls. In 8-9 month old infants, increased breastfeeding duration was also correlated with reduced REM sleep, and children with exclusive breastfeeding had less SDB by questionnaire. Finally, exclusively formula-fed infants were diagnosed with "primary snoring" more often than those with any amount of breastfeeding.

Conclusions: The findings in this dissertation reveal associations between breastfeeding, SDB, and REM sleep with quantifiable brain development. These results support the idea that certain cognitive effects associated with SDB and infant feeding methods may have common underlying brain changes that can help explain these observed differences in cognition.

Keywords: Sleep-Disordered Breathing, Breastfeeding, Rapid Eye Movement (REM), White Matter, Brain Development, Magnetic Resonance Imaging (MRI), Polysomnography (PSG) 


\section{Acknowledgements}

There are many people to whom I am most grateful for their assistance during my graduate training. I would firstly like to thank my primary advisor, Dr. Hawley MontgomeryDowns, for all of her support and assistance not only with this dissertation but also many other projects in the lab. Without the training that I received from her, and my friend and colleague Margeaux, this dissertation would not be possible. Hawley not only accepted me into the lab under difficult circumstances, but also provided me with the encouragement needed to learn and complete this project, alongside numerous valuable suggestions. She has taught me everything I know about sleep. I would also like to thank Dr. Julie Brefczynski-Lewis for her advisory role not only in this project but also other projects pre-dating my dissertation research.

I would also like my first primary advisor, Dr. Stanislaw Majewski, for all his support and assistance early in my graduate career and the opportunities which he provided and continues to provide. He, along with Dr. Alexander Stolin (Sasha), have taught me everything I know about positron emission tomography (PET) and image processing. Early research we all took part in (along with Julie) was an integral piece in winning later grant funding.

I would like to thank my graduate student friends and colleagues Margeaux, Colleen, Paula, Caitlin, Billie, and Neetu for their continued assistance through this graduate school endeavor, among others. For this dissertation in particular, I must thank Paula and Caitlin for certain procedures and reliability measurements needed for this project, Billie for double checking of data, and Margeaux for assistance in writing and research suggestions. 
I thank my entire committee for their outstanding input during not only the formative stages of this dissertation, but also during the proposal defense and beyond. Their suggestions were not only instrumental in bettering the project, but were also given in a way that was encouraging and fulfilling.

Finally, I would like to thank my family for their love and support during graduate school. Their belief in my abilities and pride in me pushed me through the more difficult parts of the PhD process. I would like to thank all my friends, Shannon, John, Matt, Zack, Brad, Kevin, Andy, and everyone else for their encouragement. 


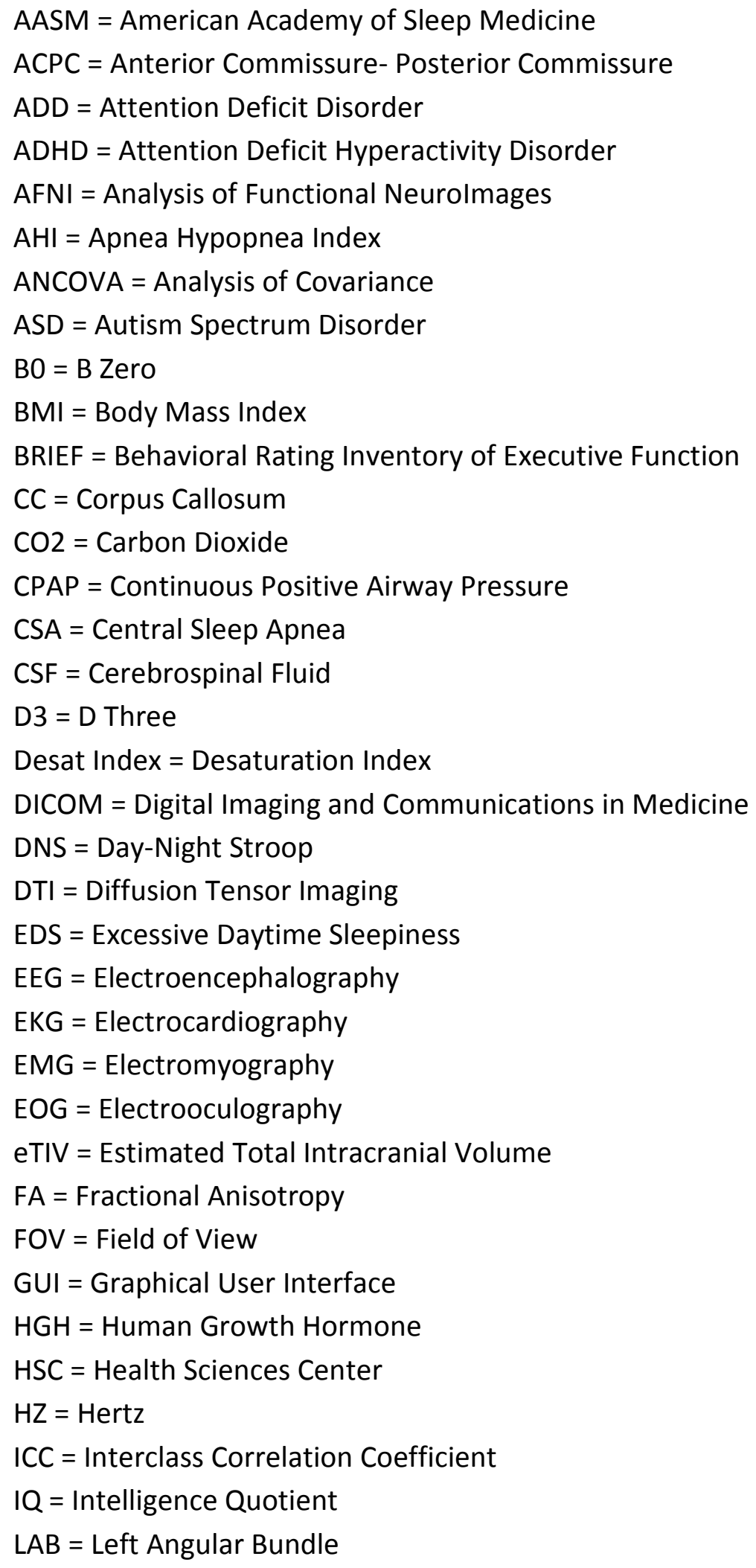




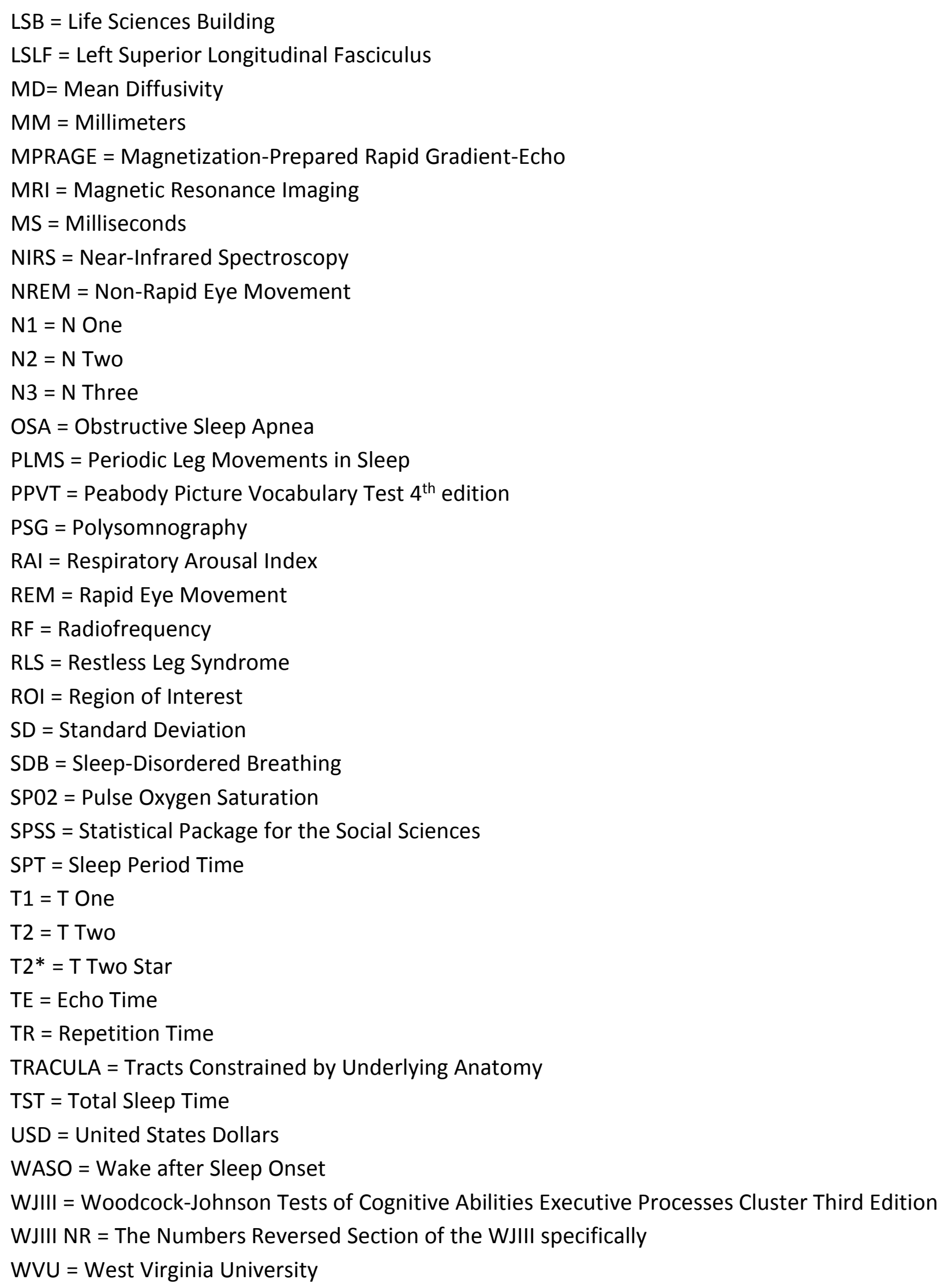


Table of Contents

Acknowledgements

iv-v

Acronyms and Abbreviations

vi-vii

Table of Contents

viii-x

Figures

$x i-x x$

Tables

xxi-xxxiv

1.0 Introduction

$1-40$

1.1 Preamble

1

1.2 Normative Sleep

2-5

1.2.1 Infant Sleep

$2-3$

1.2.2 Pediatric Sleep

3-4

1.2.3 Adult Sleep

4-5

1.3 Disordered Sleep

5-20

1.3.1 Overview of Sleep Disorders

6-15

1.3.2 Sleep-Disordered Breathing in Adults

$15-16$

1.3.3 Sleep-Disordered Breathing in Children

16-19

1.3.4 Sleep-Disordered Breathing in Infants

$19-20$

1.4 Infant Feeding Methods

20-26

1.4.1 Infant Feeding Methods and Sleep

21-22

1.4.2 Breastfeeding and Sleep-Disordered Breathing

$22-23$

1.4.3 Breastfeeding and Brain Development

23-25

1.4.4 Breastfeeding and Neurocognitive Outcomes

$25-26$

1.5 Significance of White Matter in the Brain

26-31

1.5.1 Anatomy and Function

27-28

1.5.2 Imaging and Methodology

28-29

1.5.3 Association with Neurocognitive and Behavioral Outcomes 29-30

1.5.4 Association with Developmental Disorders 30-31

1.6 Rapid Eye Movement (REM) Sleep and Brain Development

31-35

1.7 Putting it All Together: Breastfeeding, Sleep-Disordered Breathing, and White Matter Development

1.7.1 Specific Aim 1

38-39

1.7.2 Specific Aim 2

39

1.7.3 Specific Aim 3

$39-40$

2.0 Methods

40-71

2.1 General Methods

40-48

2.1.1 Magnetic Resonance Imaging (MRI)

40-45

2.1.2 Diffusion Tensor Imaging (DTI)

45-46 
2.1.3 Polysomnography (PSG) 46-48

2.2 Specific Aim $1 \quad$ 48-61

2.2.1 Description of Procedures 48-53

2.2.2 Data Processing 53-61

$\begin{array}{lr}2.3 \text { Specific Aim } 2 & \text { 61-67 }\end{array}$

2.3.1 Description of Procedures 61-63

2.3.2 Data Processing 64-67

2.4 Specific Aim $3 \quad$ 67-71

2.4.1 Description of Procedures Part $1 \quad$ 67-69

2.4.2 Data Processing Part $1 \quad 69$

2.4.3 Description of Procedures Part $2 \quad$ 70-71

2.4.4 Data Processing Part 2 71

$\begin{array}{ll}3.0 \text { Results } & 71-97\end{array}$

3.1 Specific Aim $1 \quad 71-80$

3.1.1 Hypotheses $\quad$ 71-72

3.1.2 Preliminary Analysis $\quad$ 72-75

3.1.2.1 Participant Characteristics

3.1.2.2 Normality and Outliers $\quad$ 73-74

3.1.2.3 SDB Characterization $\quad$ 74-75

3.1.2.4 CC Corrections $\quad 75$

$\begin{array}{ll}\text { 3.1.3 Aim } 1 \text { Results } & \text { 75-80 }\end{array}$

3.1.3.1 Hypothesis 1a $\quad$ 75-78

3.1.3.2 Hypothesis $1 b \quad$ 78-79

3.1.3.3 Supplemental Analyses $\quad$ 79-80

3.2 Specific Aim $2 \quad 80-88$

3.2.1 Hypotheses $\quad 80-81$

3.2.2 Preliminary Analysis 81-83

3.2.2.1 Participant Characteristics $\quad$ 81-82

3.2.2.2 Normality and Outliers $\quad$ 82-83

3.2.3 Aim 2 Results 83-88

3.2.3.1 Hypothesis $2 \mathrm{a} \quad 83$

3.2.3.2 Hypothesis $2 b \quad$ 83-85

3.2.3.3 Hypothesis $2 \mathrm{c} \quad 86$

3.2.3.4 Hypothesis $2 \mathrm{~d} \quad$ 86-87

3.2.3.5 Supplementary Analyses $\quad$ 87-88

3.3 Specific Aim $3 \quad$ 88-97

3.3.1 Hypotheses 88

3.3.2 Preliminary Analysis $\quad$ 88-92 
3.3.2.1 Participant Characteristics $\quad 88-90$

3.2.2.1.1 Hypothesis 3a Participants

3.2.2.1.2 Hypothesis 3b Participants $\quad$ 89-90

3.3.2.2 Hypothesis 2a Normality and Outliers $\quad 90-91$

3.3.2.3 Hypothesis 2b Normality and Outliers $\quad 91-92$

3.3.3 Aim 3 Results $\quad$ 92-97

3.3.3.1 Hypothesis 3a 92-93

3.3.3.2 Hypothesis 3b 93-97

$\begin{array}{lr}4.0 \text { Discussion } & \text { 97-116 }\end{array}$

4.1 Specific Aim $1 \quad$ 97-101

4.1.1 Hypothesis $1 \mathrm{a} \quad 98$

4.1.2 Hypothesis $1 b \quad 99-101$

4.2 Specific Aim $2 \quad$ 101-105

4.2.1 Hypothesis 2a 101-102

4.2.2 Hypothesis $2 b \quad 102-104$

4.2.3 Hypothesis 2c 104

4.2.4 Hypothesis 2d 104-105

4.3 Specific Aim $3 \quad$ 105-108

4.3.1 Hypothesis 3a 105

4.3.2 Hypothesis 3b 105-108

4.4 Summary of All Aims $\quad$ 108-112

4.5 Limitations $\quad$ 112-115

4.6 Future Directions $\quad$ 115-116

Bibliography $\quad$ 117-130

$\begin{array}{lr}\text { Appendices } & 131-172\end{array}$ 


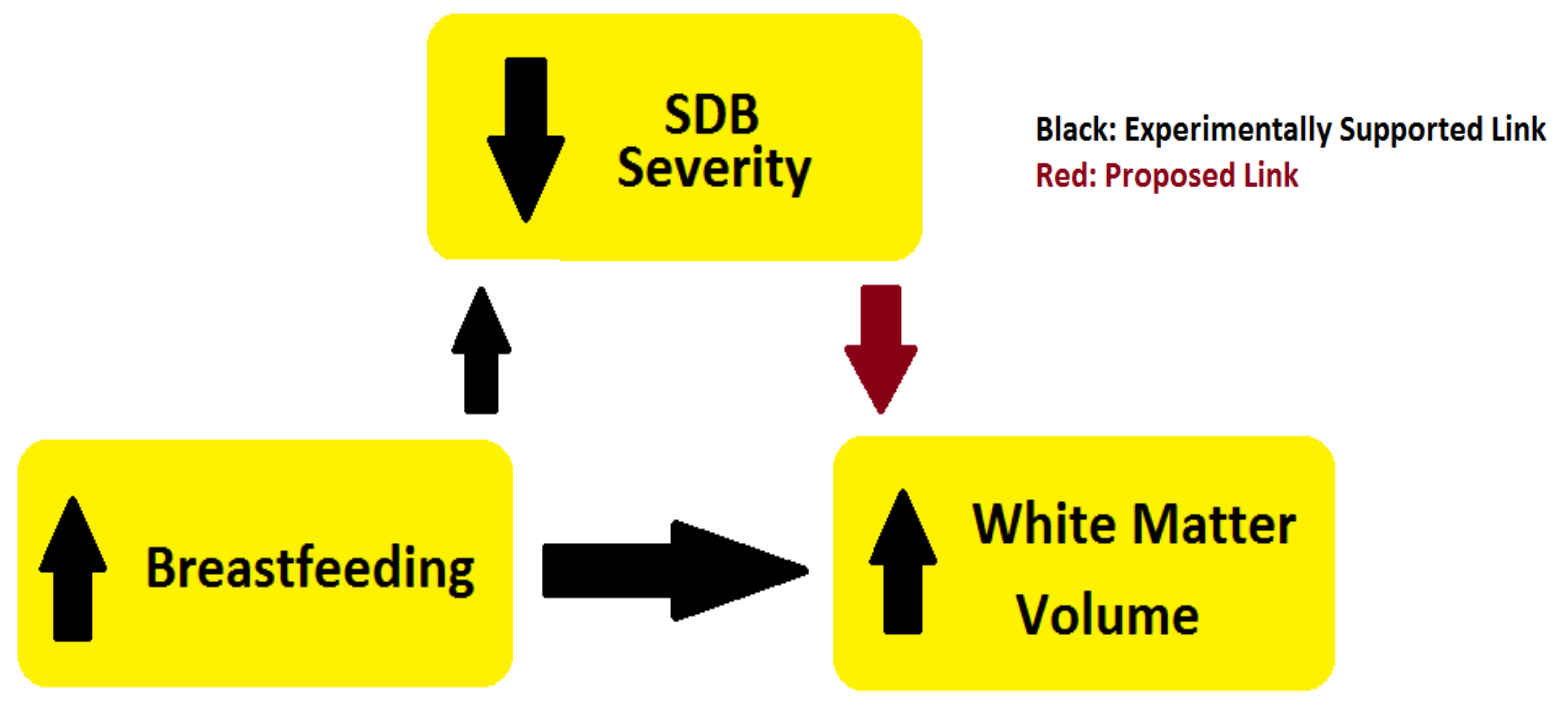

Fig 1. Simple model of my proposed dissertation.

The main direct effect of breastfeeding was predicted to involve white matter volume through previously published mechanisms, such as nutrition, social factors, immune support, and other miscellaneous factors. However, at least a portion of the variance in this relationship should be attributed to sleep. I proposed that breastfeeding would be associated with reduced SDB severity, and that children with reduced SDB severity (or healthy) would have greater white matter volume than children with greater SDB severity, indicative of healthy maturation of neural connectivity. 


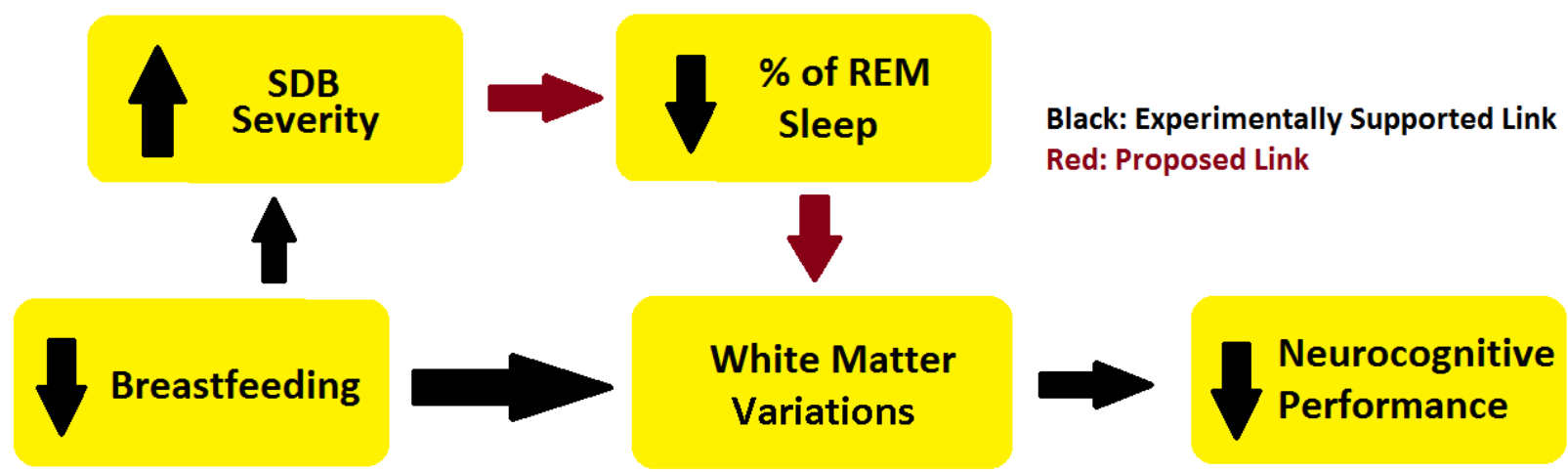

Fig 2. Full model of my proposed dissertation.

In addition to what was stated in the simple model, the full model explains the mechanism by which I think sleep is influencing brain development. An increased severity of SDB may decrease the quality and quantity of REM sleep, resulting in decreased stimulation of neural circuits during REM sleep (ontogenetic hypothesis). This may ultimately produce the observed phenotype of decreased white matter volume and quality, which could help explain decreases in neurocognitive performance observed in children with SDB. 


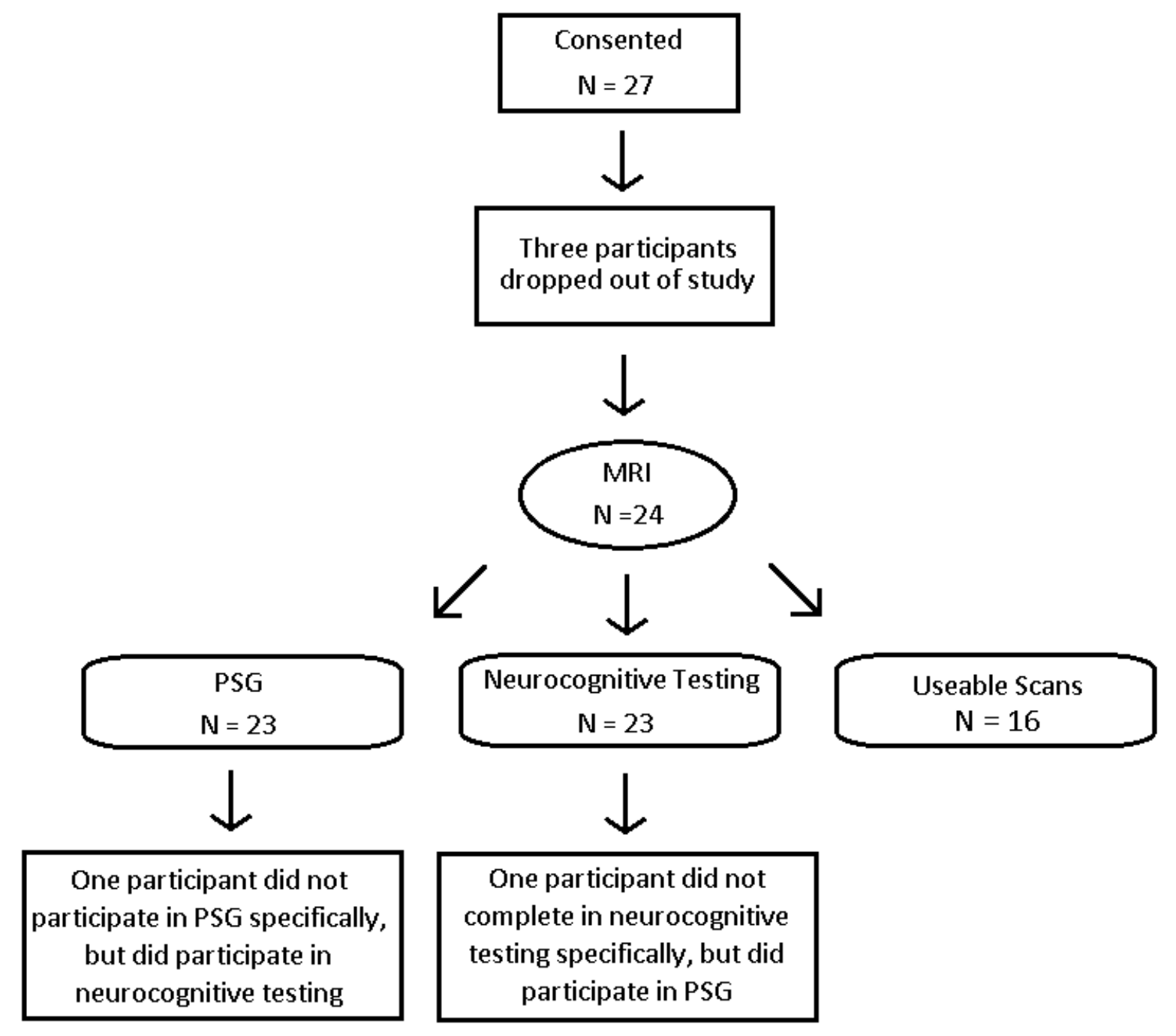

Fig 3. Flow chart for participants in each stage of aim 1. 


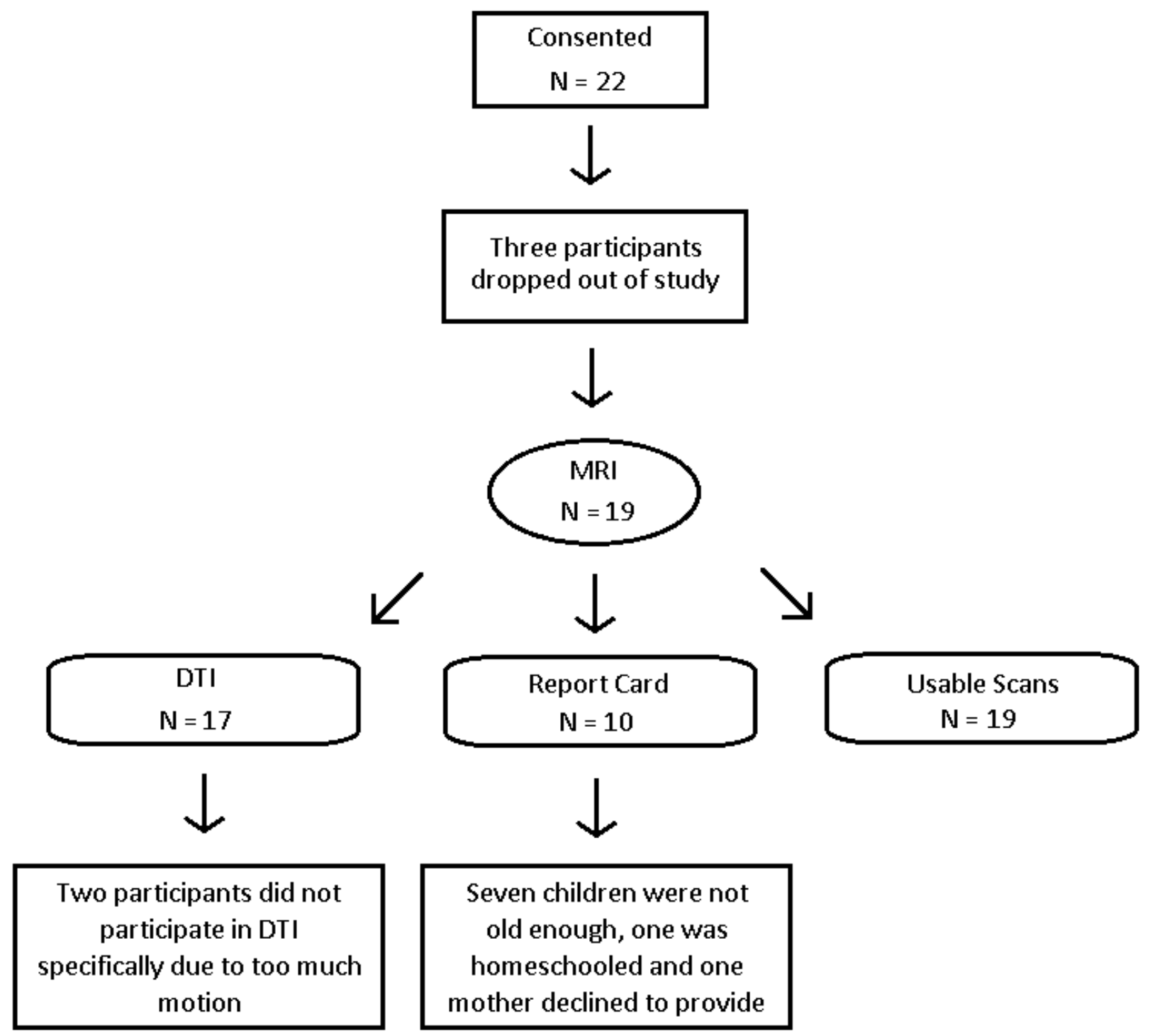

Fig 4. Flow chart for participants in each stage of aim 2. 


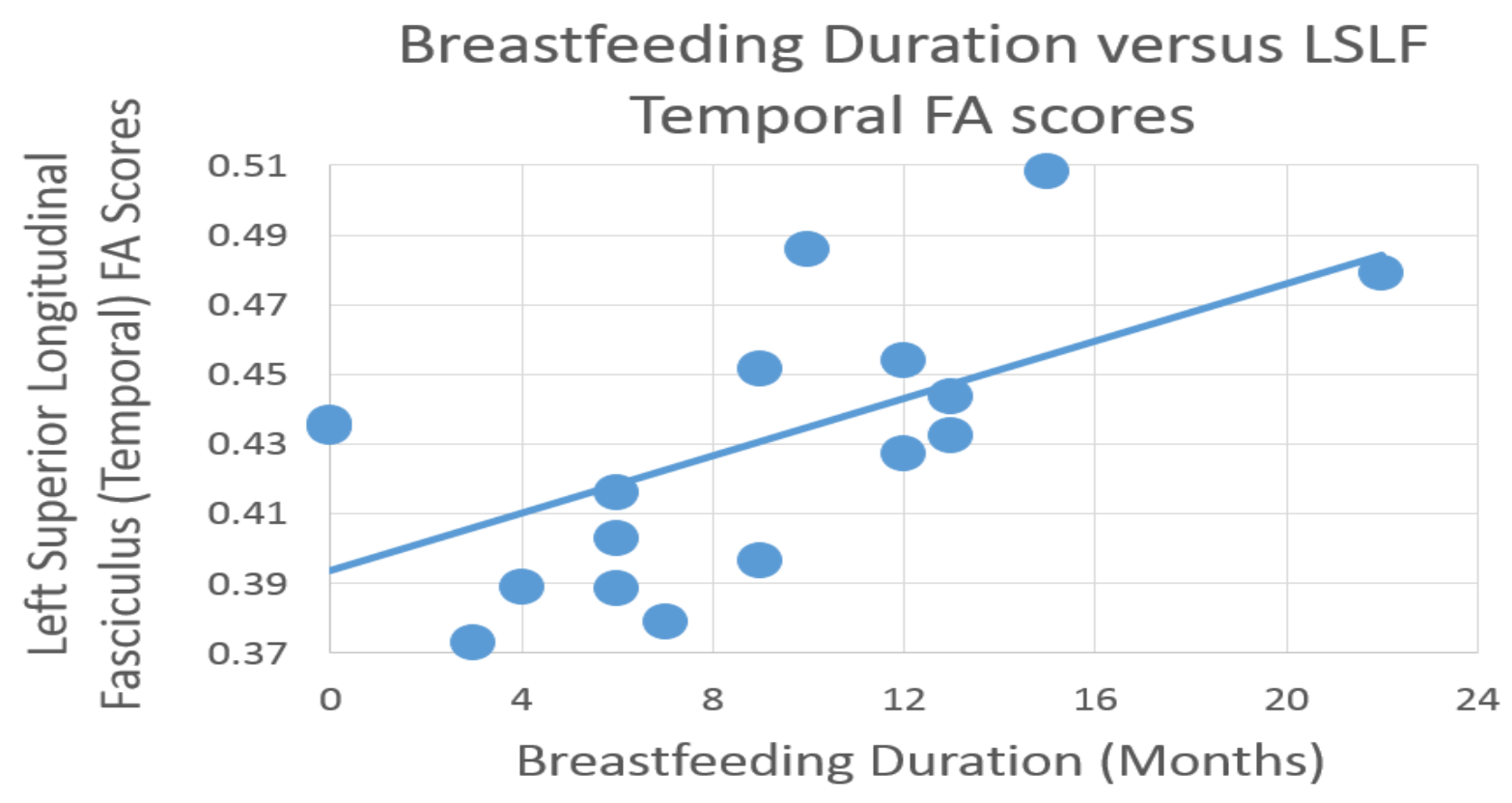

Breastfeeding Duration versus LAB FA

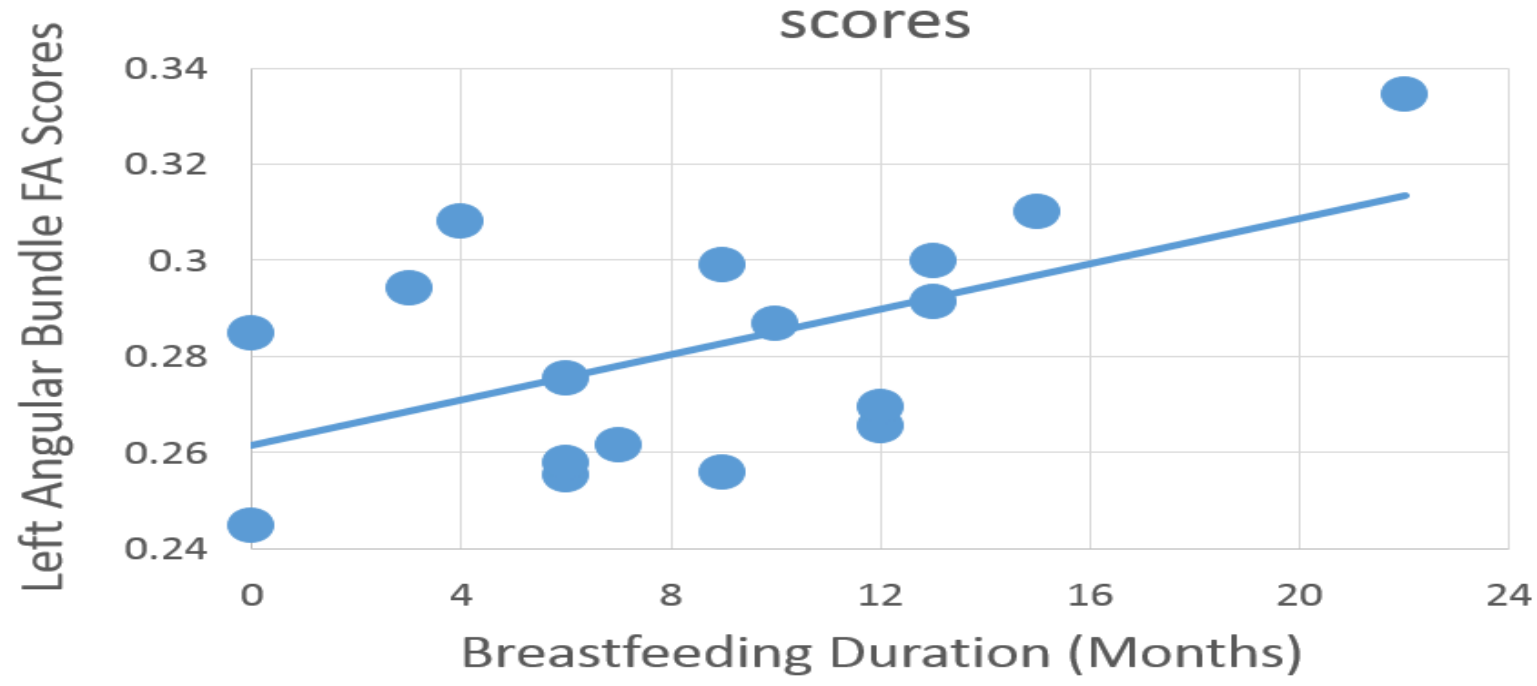

Fig 5. Simple scatter plot between breastfeeding duration and fractional anisotropy (FA) scores for the left superior longitudinal fasciculus (LSLF; temporal portion) and left angular bundle (LAB).

(Top) Association between breastfeeding duration and LSLF FA scores. A stepwise multiple linear regression revealed that breastfeeding was the only significant predictor $\left(p=0.019 ; R^{2}=0.354\right)$. (Bottom) Simple scatter plot between breastfeeding duration and FA scores for the LAB. A stepwise multiple linear regression revealed that breastfeeding was the only significant predictor $\left(p=0.034 ; R^{2}=0.301\right)$. 


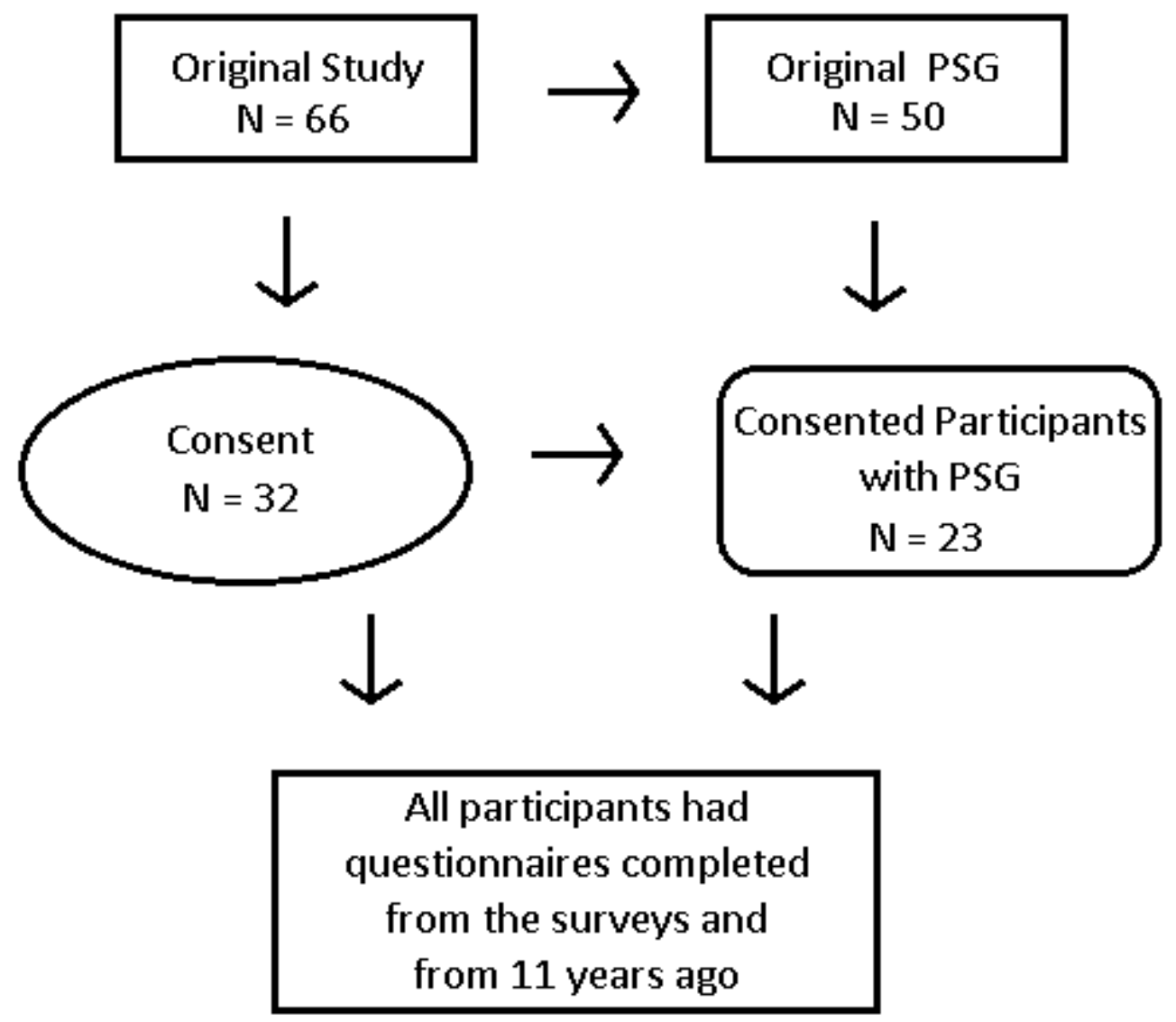

Fig 6. Flow chart of participation in aim 3b. 


\section{Breastfeeding Duration Compared Against} REM Sleep in $\sim 8$ - 9 Month Old Infants

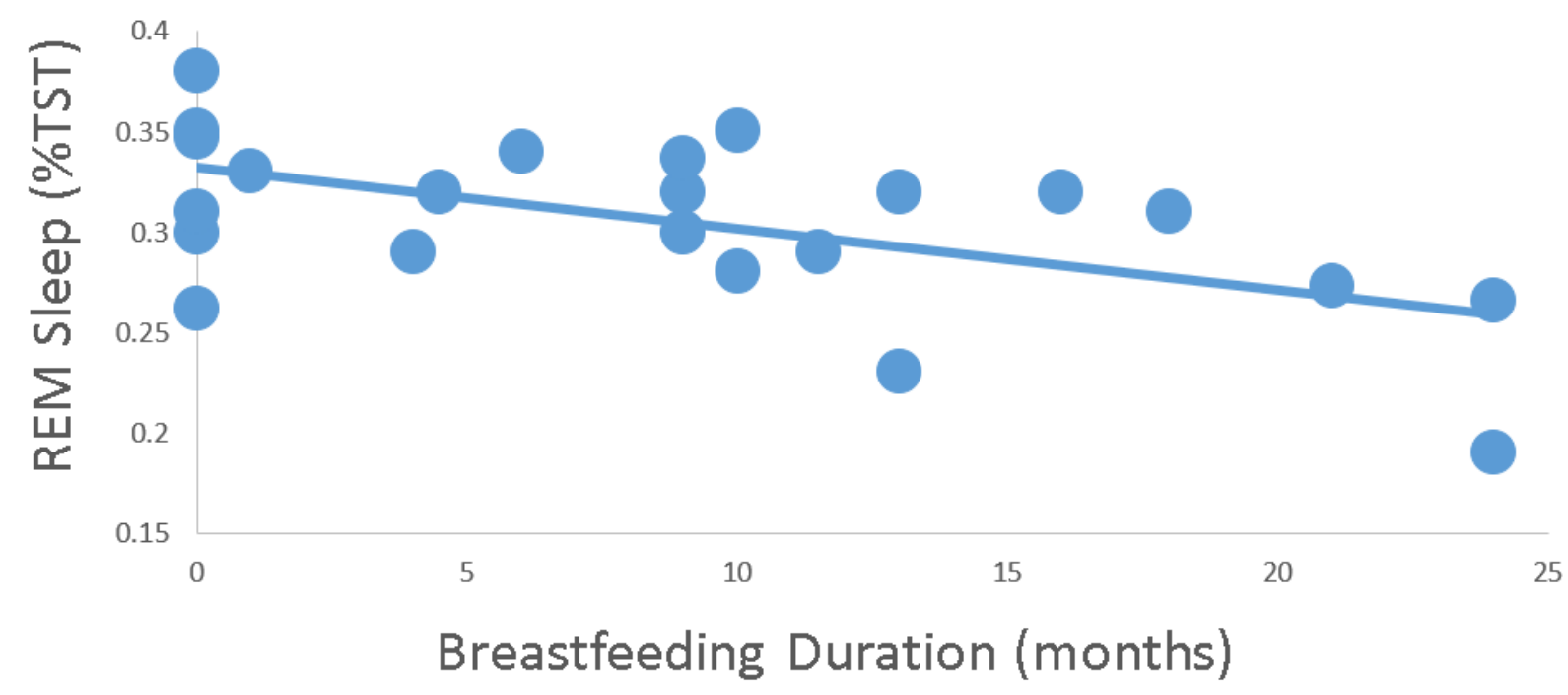

Fig 7. Simple scatter plot comparing breastfeeding duration with \% active (REM) sleep. Multiple regression revealed that even taking into account differences in maternal education and parental smoking this relationship was significant $\left(p=0.006 ; \mathrm{R}^{2}=0.325\right)$. This finding was actually in the opposite direction as the one predicted. 


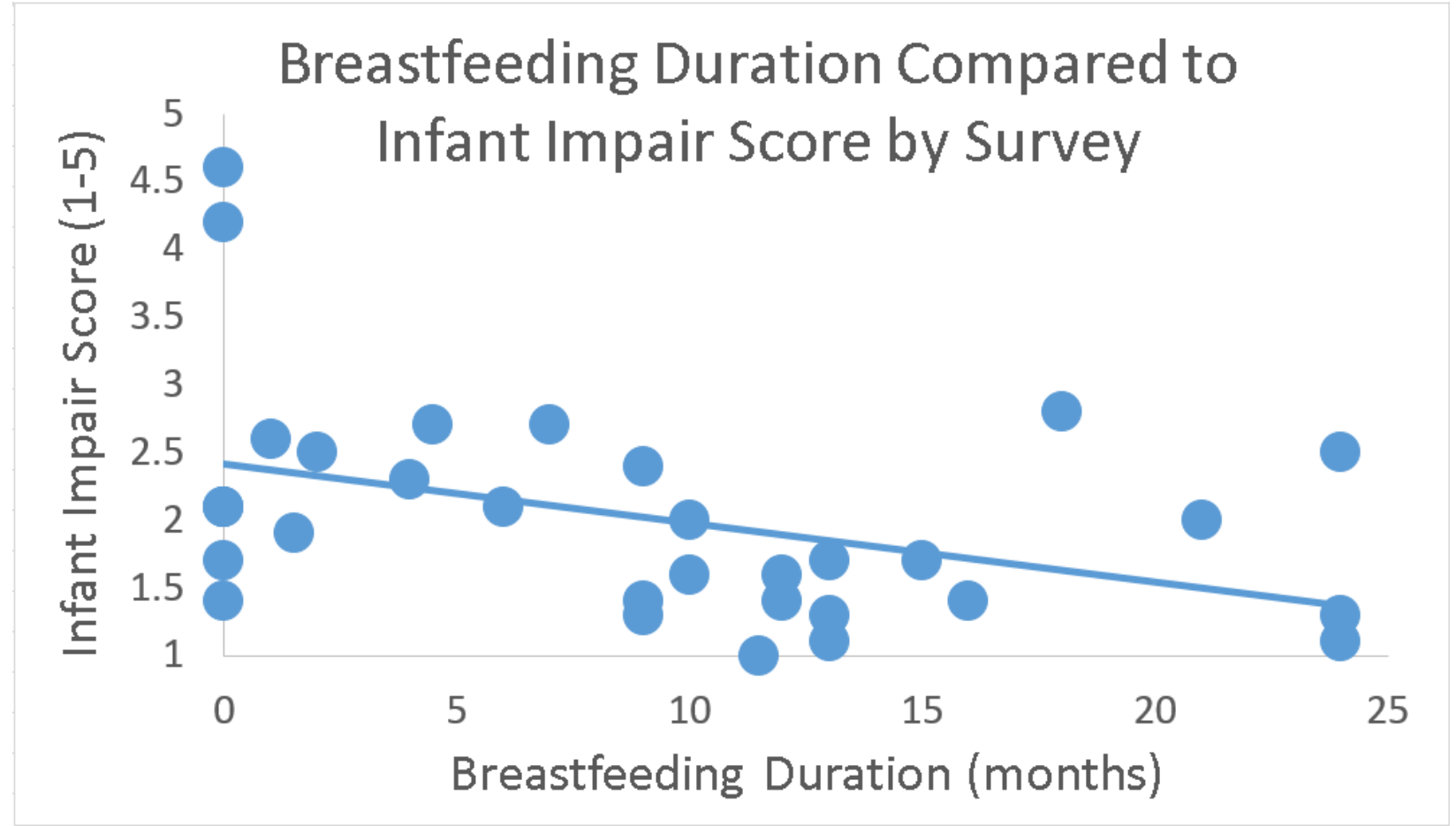

Fig 8. Scatter plot depicting breastfeeding duration against infant sleep impairment score. Controlling for parental smoking, breastfeeding duration was significantly negatively correlated with transformed impairment scores $(p=0.016)$, but not after inclusion of maternal education. The plotted data points are not transformed in this figure. 


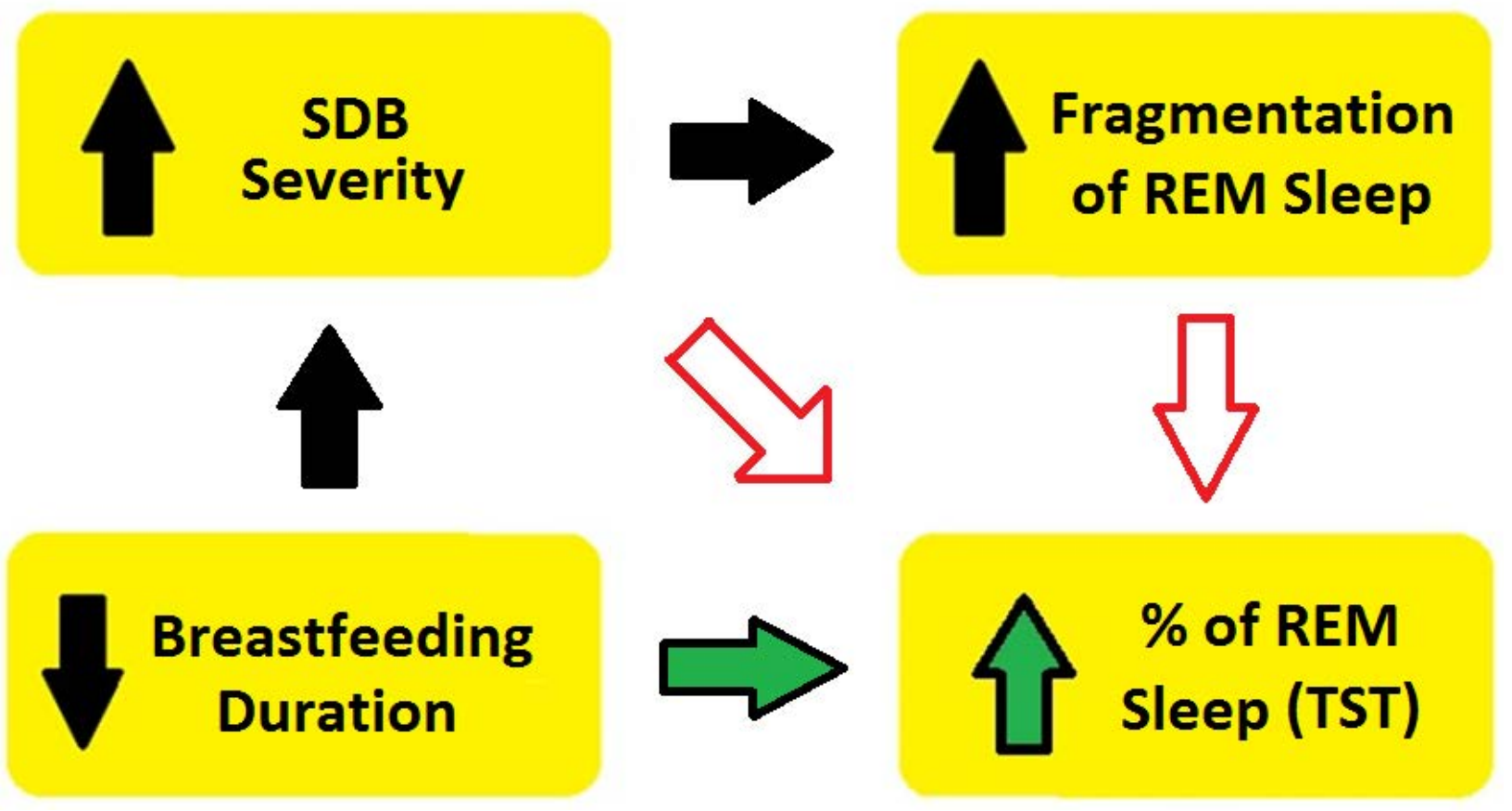

Fig 9. Summary of results from Specific Aim 3b.

Black arrows indicate significant results found in this dissertation, which are also supported by the literature. Green arrows indicate significant results found in this dissertation that, to my knowledge, have not been reported in previous literature specifically. Red empty arrows indicate a theoretical relationship which is not supported by either this dissertation or the literature, at least not in early development (childhood or infancy). 


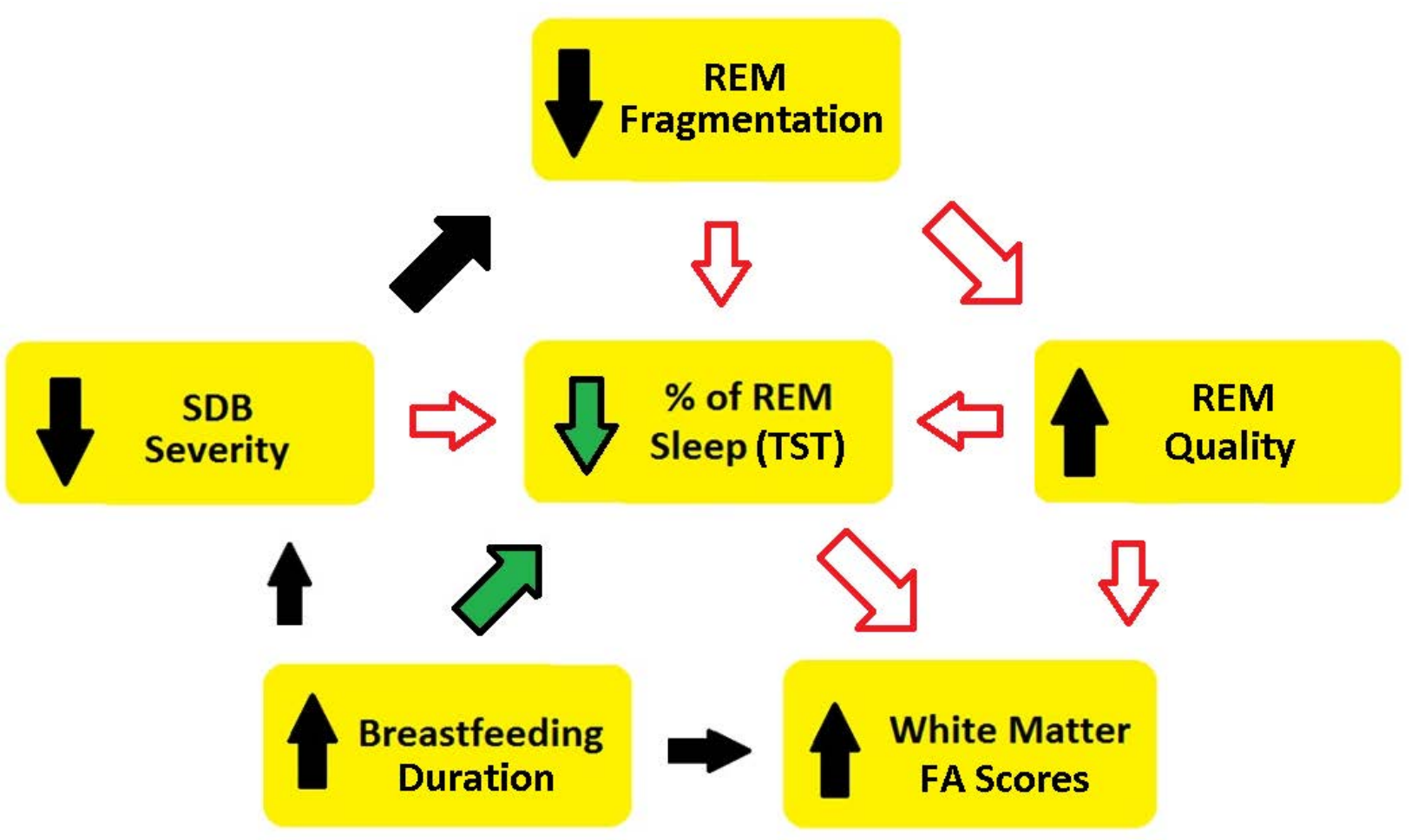

Fig 10. Summary of results from this dissertation.

Black arrows indicate significant results found in this dissertation, which are also supported by the literature. Green arrows indicate significant results found in this dissertation that, to my knowledge, have not been reported in previous literature specifically. Red empty arrows indicate a theoretical relationship which is not supported by either this dissertation or the literature, at least not in early development (childhood or infancy). 
Table 1. Demographics for aim 1.

\begin{tabular}{|c|c|c|}
\hline Demographic & Value & $\mathbf{n}$ \\
\hline Age, Mean (SD) & $6.8(1.5)$ years & 24 \\
\hline Sex & $37.5 \%$ female & 24 \\
\hline Annual Income, Median & 28,500 USD & 16 \\
\hline Maternal Education, Mean (SD) & $13.9(2.8)$ years & 23 \\
\hline Parental Smoking & $52.2 \%$ & 23 \\
\hline BMI, Mean (SD) & $19.1(5.9)$ & 22 \\
\hline Diagnosis of ADD/ADHD & $54.2 \%$ & 24 \\
\hline Exclusive Breastfeeding & $12.5 \%$ & 24 \\
\hline Exclusive Formula Feeding & $50.0 \%$ & 24 \\
\hline Breastfeeding Duration, Mean (SD) & $4.4(6.0)$ months & 24 \\
\hline
\end{tabular}


Table 2. Pearson correlations between breastfeeding variables, sleep variables, and CC size.

\begin{tabular}{|c|c|c|c|c|}
\hline & Breast_Duration & AHI & SDB_Profile & CC_Norm \\
\hline Method & $0.829 * * *$ & -0.166 & -0.140 & -0.153 \\
\hline & 24 & 23 & 23 & 16 \\
\hline Breast_Duration & & & & -0.166 \\
\hline & & -0.174 & -0.120 & 16 \\
\hline AHI & & 23 & 23 & 0.159 \\
\hline & & & & 15 \\
\hline SDB_Profile & & & & $-0.894 * *$ \\
\hline
\end{tabular}

${ }^{* * *} p<0.001$. Feeding method ( $1=$ formula, $2=$ both, $3=$ breast); breastfeeding duration refers to the duration of breastfeeding until completely weaned; CC volume (CC_Norm) refers to the total volume divided by the normalization factor. In order to look at SDB both holistically (SDB_Profile) and traditionally (AHI only), both were included. The sample size in each correlation is indicated beneath the Pearson value. The values in this table do not include correction for covariates. 
Table 3. Pearson correlations for neurocognitive measures and feeding methods, sleep outcomes, and CC volume.

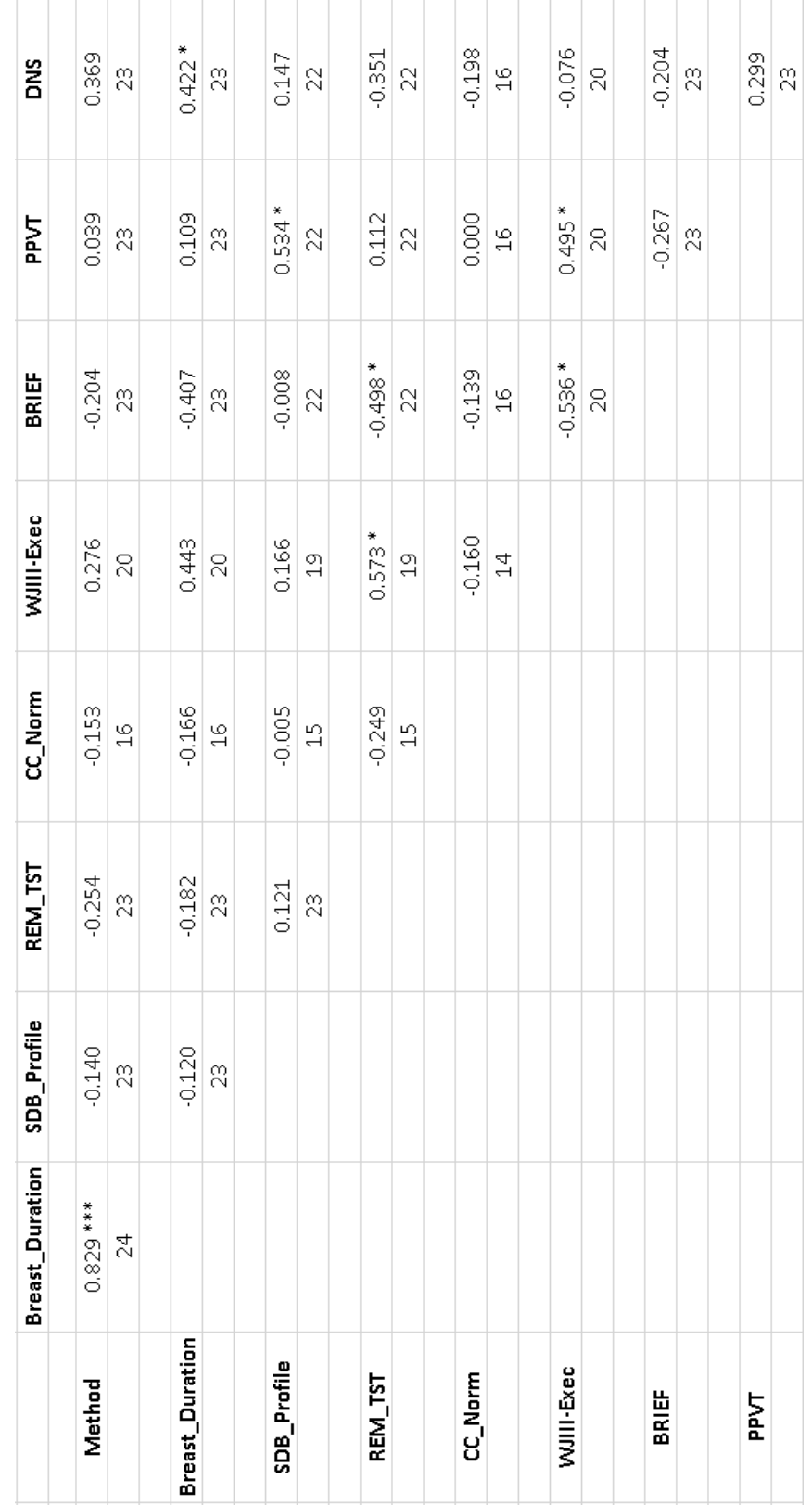

$* * * p<0.001 ; * p<0.05$. Significant correlations were detected between breastfeeding duration and scores on the WJIII_Exec and Day-Night Stroop, between SDB_Profile scores and the PPVT, and between REM sleep time and scores on the WJIII_Exec and the BRIEF. For all neurocognitive measures included here, a higher score is more desirable. The sample size for each correlation is included beneath the Pearson value. 
Table 4. Mean (SD) values for participants who were ever breastfed or who were exclusively formula fed.

\begin{tabular}{|c|c|c|c|c|}
\hline & Exclusively Formula-Fed & Any Breastfeeding & p value & Effect Size \\
\hline SDB_Profile & $1.1(0.75 ; \mathrm{n}=12)$ & $0.94(0.62 ; \mathrm{n}=11)$ & 0.828 & Cohen's $d=0.169$ \\
\hline AHI & $4.4(6.0 ; \mathrm{n}=12)$ & $3.0(2.8 ; \mathrm{n}=11)$ & 0.712 & Cohen's $d=0.306$ \\
\hline \% REM & $16.7(5.4 ; \mathrm{n}=12)$ & $15.0(6.0 ; \mathrm{n}=11)$ & 0.482 & Cohen's $d=0.299$ \\
\hline CC_Total & $6543(1494 ; \mathrm{n}=6)$ & $6941(1438 ; \mathrm{n}=10)$ & 0.605 & Hedges' $g=0.273$ \\
\hline CC_Central & $1360(404 ; \mathrm{n}=6)$ & $1453(299 ; \mathrm{n}=10)$ & 0.605 & Hedges' $g=0.273$ \\
\hline WJ-III & $39.4(18.4 ; \mathrm{n}=9)$ & $50.3(22.0 ; \mathrm{n}=11)$ & 0.255 & Cohen's $d=0.534$ \\
\hline Income & $21380(16727 ; \mathrm{n}=10)$ & $40075(27641) ; \mathrm{n}=8)$ & 0.095 & Cohen's $d=0.818$ \\
\hline
\end{tabular}


Table 5. Mean (SD) values for participants who had an AHI $>=1$ or an $A H I<1$.

\begin{tabular}{|c|c|c|c|c|}
\hline & $\mathrm{AHI}>=1$ & $\mathrm{AHI}<1$ & $p$ value & Effect Size \\
\hline SDB_Profile & $1.2(0.61 ; n=17)$ & $0.36(0.40 ; n=6)$ & 0.001 & Hedges' $g=1.527$ \\
\hline $\mathrm{AHI}$ & $4.8(5.0 ; n=17)$ & $0.60(0.32 ; n=6)$ & 0.000 & Hedges' $g=0.966$ \\
\hline$\%$ REM & $16.8(5.8 ; n=17)$ & $13.3(4.8 ; n=6)$ & 0.195 & Hedges' $g=0.636$ \\
\hline CC_Total & $6481(1274 ; n=10)$ & $7418(1790 ; n=5)$ & 0.260 & Hedges' $g=0.645$ \\
\hline CC_Central & $1336(297 ; n=10)$ & $1597(397 ; n=5)$ & 0.174 & Hedges' $g=0.789$ \\
\hline WJ-III & $48.4(18.8 ; n=14)$ & $32.0(21.8 ; n=5)$ & 0.125 & Hedges' $g=0.839$ \\
\hline Income & $34223(24429 ; n=14)$ & $13821(11056 ; n=4)$ & 0.130 & Hedges' $g=0.905$ \\
\hline
\end{tabular}


Table 6. Group differences between children with at least one parent who smoked and children with non-smoking parents.

\begin{tabular}{|c|c|c|}
\hline & Parent who Smokes & No Parent who Smokes \\
\hline Breastfeeding Duration*, Mean (SD) & $2.3(4.4 ; \mathrm{n}=12)$ months & $7.8(6.8 ; \mathrm{n}=11)$ months \\
\hline SDB_Profile, Mean (SD) & $1.1(0.81 ; \mathrm{n}=12)$ & $0.80(0.34 ; \mathrm{n}=10)$ \\
\hline AHI, Mean (SD) & $4.4(6.1 ; \mathrm{n}=12)$ & $2.3(1.5 ; \mathrm{n}=10)$ \\
\hline CC_Total, Mean (SD) & $6146(1415 ; \mathrm{n}=8) \mathrm{mm}^{3}$ & $7346(1238 ; \mathrm{n}=7) \mathrm{mm}^{3}$ \\
\hline CC_Central*, Mean (SD) & $1246(288 ; \mathrm{n}=8) \mathrm{mm}^{3}$ & $1584(315 ; \mathrm{n}=7) \mathrm{mm}^{3}$ \\
\hline BRIEF*, Mean (SD) & $93.8(4.3 ; \mathrm{n}=11)$ & $79.3(17.5 ; \mathrm{n}=11)$ \\
\hline Income, Mean (SD) & $22534(14502 ; \mathrm{n}=9)$ USD & $28950(18239 ; \mathrm{n}=8)$ USD \\
\hline$* p<0.05$ & & \\
\hline
\end{tabular}


Table 7. Demographics for participants in aim 2.

\begin{tabular}{|c|c|c|}
\hline Demographic & Value & $\mathbf{n}$ \\
\hline Age, Mean (SD) & $6.4(1.6)$ years & 19 \\
\hline Sex & $36.8 \%$ female & 19 \\
\hline Annual Income, Median & 80,000 USD & 16 \\
\hline Maternal Education, Mean (SD) & $17.4(1.9)$ years & 19 \\
\hline Parental Smoking & $15.8 \%$ & 19 \\
\hline Diagnosis of ADD/ADHD & $5.3 \%$ & 19 \\
\hline Exclusive Breastfeeding & $36.8 \%$ & 19 \\
\hline Exclusive Formula Feeding & $10.5 \%$ & 19 \\
\hline Breastfeeding Duration, Mean (SD) & $9.9(7.1)$ months & 19 \\
\hline
\end{tabular}


Table 8. Mean (SD) values between the sleep-disordered breathing population in aim 1 and the healthy population in aim 2.

\begin{tabular}{|c|c|c|}
\hline Category & SDB & Healthy \\
\hline Age & $6.6(1.7)$ & $6.4(1.6)$ \\
\hline Sex & $40 \%$ female & $37 \%$ female \\
\hline Maternal Age & $35.3(7.1)$ & $34.6(4.7)$ \\
\hline Maternal Education & $13.8(3.1)$ & $17.4(1.9)$ \\
\hline Annual Income & $30000($ median) & 80000 (median) \\
\hline Apnea-Hyponea Index & $4.5(3.0)$ & - \\
\hline CC Total Volume AFNI & $6481(1274 ; \mathrm{n}=10)$ & $7832(866 ; \mathrm{n}=19)$ \\
\hline CC Total Volume Freesurfer & $6649(532 ; \mathrm{n}=4)$ & $7241(828 ; \mathrm{n}=18)$ \\
\hline CC Central Volume Freesurfer & $820(85)$ & $936(128)$ \\
\hline
\end{tabular}

Apnea-hypopnea index included is for subjects with AHIs greater than one. Although the healthy population did have larger CCS on average, the difference was not significant in the case of AFNI ( $p=0.155$; hedges' $\mathrm{g}=0.586)$ or Freesurfer $(p=0.217$; hedges' $\mathrm{g}=0.700)$ when correcting by the normalization factor. Reported volumes are in cubic millimeters. 
Table 9. General demographics and feeding information for participants in aim 3 part 1.

\begin{tabular}{|c|c|c|}
\hline Demographic & Value & $\mathbf{n}$ \\
\hline Age, Mean (SD) & & \\
\hline Sex & $34.8(16.5)$ months & 14 \\
\hline Maternal Education, Mean (SD) & $57 \%$ female & 14 \\
\hline Exclusive Breastfeeding & $16.1(2.5)$ years & 13 \\
\hline Exclusive Formula Feeding & $35.7 \%$ & 14 \\
\hline Breastfeeding Duration, Mean (SD) & $9.5(8.4)$ months & 14 \\
\hline
\end{tabular}


Table 10. Mean (SD) values for summary sleep measures obtained through PSG.

\section{Sleep Statistic}

Total Sleep Time, Minutes (SD) 333.9 (115.9) 14

Awakenings, Count Number (SD) WASO, \% Sleep Period Time (SD)

N2, \% Total Sleep Time (SD)

N3, \% Total Sleep Time (SD)

REM, \% Total Sleep Time (SD)
Mean Value

$15.4(8.4) \quad 14$

$3.2(2.4) \quad 14$

$41.8(8.5) \quad 14$

$34.2(11.3) \quad 14$

$22.1(7.2) \quad 14$ 
Table 11. General demographics and breastfeeding information for participants who returned the mailed surveys.

\begin{tabular}{|c|c|c|}
\hline Demographic & Value & $\mathbf{n}$ \\
\hline Sex & & \\
\hline Annual Income, Median & $40.6 \%$ female & 32 \\
\hline Maternal Education, Mean (SD) & 90,000 USD & 30 \\
\hline Parental Smoking & $16.8(2.6)$ years & 32 \\
\hline Exclusive Breastfeeding & $22.6 \%$ & 31 \\
\hline Exclusive Formula Feeding & $34.4 \%$ & 32 \\
\hline Breastfeeding Duration, Mean (SD) & $9.0(7.7)$ months & 32 \\
\hline
\end{tabular}


Table 12. PSG metrics for participants in aim 3b.

\begin{tabular}{|c|c|c|}
\hline Sleep Statistic & Value & $\mathbf{n}$ \\
\hline WASO, Mean \% Sleep Period Time (SD) & $13.1(6.2)$ & 23 \\
\hline Active, Mean \% Total Sleep Time, (SD) & $30.5(4.3)$ & 23 \\
\hline RAI Active, Mean (SD) & $0.84(1.5)$ & 21 \\
\hline Snoring Arousal Index Active, Mean (SD) & $0.54(0.98)$ & 21 \\
\hline Snoring on PSG & $52.2 \%$ & 23 \\
\hline Primary Snoring & $38.1 \%$ & 21 \\
\hline
\end{tabular}


Table 13. Mean (SD) values for various demographics and comparable variables for breastfeeding groups compared by $t$-tests, Mann-Whitney $U$ tests, and Pearson's Chi-Squared test.

\begin{tabular}{|c|c|c|c|c|}
\hline & Exclusive Breastfed & Any Formula & p-value & Hedges' g \\
\hline Sex (\% Female) & $27.3(\mathrm{n}=11)$ & $47.6(\mathrm{n}=21)$ & 0.266 & - \\
\hline Annual Household Income (Med) & $90000(\mathrm{n}=10)$ & $85844(\mathrm{n}=20)$ & - & - \\
\hline Maternal Education & $18.2(2.0, \mathrm{n}=11)$ & $16.1(2.6, \mathrm{n}=21)$ & 0.029 & 0.869 \\
\hline Maternal Age & $30.9(3.2, \mathrm{n}=7)$ & $30.0(5.9, \mathrm{n}=15)$ & 0.667 & 0.172 \\
\hline Parental Smoke (\%) & $0.0(\mathrm{n}=10)$ & $33.3(\mathrm{n}=21)$ & 0.038 & - \\
\hline \% Active (REM) of TST & $27.7(4.9, \mathrm{n}=8)$ & $32.1(3.12, \mathrm{n}=15)$ & 0.015 & 1.14 \\
\hline Transformed Infant Sleep Impair Score & $1.1(0.12, \mathrm{n}=11)$ & $1.3(0.14, \mathrm{n}=21)$ & 0.002 & 1.50 \\
\hline Active RAI & $0.29(0.41, \mathrm{n}=8)$ & $1.0(1.7, \mathrm{n}=15)$ & 0.329 & 0.504 \\
\hline Snore Arousal Index during Active & $0.13(0.35, \mathrm{n}=8)$ & $0.69(1.1, \mathrm{n}=15)$ & 0.120 & 0.608 \\
\hline Snoring on PSG (\%) & $25.0(\mathrm{n}=8)$ & $66.7(\mathrm{n}=15)$ & 0.057 & - \\
\hline "Primary Snoring" (\%) & $12.5(\mathrm{n}=8)$ & $53.8(\mathrm{n}=13)$ & 0.058 & - \\
\hline
\end{tabular}

Comparisons between exclusive breastfeeding and any formula-feeding revealed significant differences in active (REM) sleep and transformed survey scores on infant sleep impairment. However, these group comparisons must be taken in light of other factors, such as differences in income, education, and possibly presence of a smoking parent. 
Table 14. Mean (SD) values for demographics and comparable variables for breast and formula feeding groups compared by $t$-tests, Mann-Whitney $U$ tests, and Pearson's ChiSquared test.

\begin{tabular}{|c|c|c|c|c|}
\hline & Exclusive Formula & Any Breastfed & p-value & Hedges' g \\
\hline Sex (\% Female) & $71.4(0.49, n=7)$ & $32.0(0.48, n=25)$ & 0.060 & - \\
\hline Annual Household Income (Med) & $51000(\mathrm{n}=6)$ & $90000(\mathrm{n}=24)$ & - & - \\
\hline Maternal Education & $14.1(1.8, \mathrm{n}=7)$ & $17.6(2.3, \mathrm{n}=25)$ & 0.001 & 1.58 \\
\hline Maternal Age & $30.5(7.3, \mathrm{n}=6)$ & $30.2(4.4, \mathrm{n}=16)$ & 0.903 & 0.057 \\
\hline Parental Smoke (\%) & $71.4(\mathrm{n}=7)$ & $8.3(\mathrm{n}=24)$ & 0.001 & - \\
\hline \% Active (REM) of TST & $32.5(4.3, \mathrm{n}=6)$ & $29.8(4.2, \mathrm{n}=17)$ & 0.185 & 0.639 \\
\hline Transformed Infant Sleep Impair Score & $1.4(0.21, \mathrm{n}=7)$ & $1.2(0.13, \mathrm{n}=25)$ & 0.046 & 1.34 \\
\hline Active RAI & $1.7(2.2, \mathrm{n}=6)$ & $0.42(0.93, \mathrm{n}=17)$ & 0.009 & 0.951 \\
\hline Snore Arousal Index during Active & $0.92(0.94, \mathrm{n}=6)$ & $0.35(0.94, \mathrm{n}=17)$ & 0.019 & 0.606 \\
\hline Snoring on PSG (\%) & $100(\mathrm{n}=6)$ & $35.3(\mathrm{n}=17)$ & 0.006 & - \\
\hline "Primary Snoring" (\%) & $80.0(\mathrm{n}=5)$ & $25.0(\mathrm{n}=16)$ & 0.027 & - \\
\hline
\end{tabular}

Comparisons between exclusive formula-feeding and any breastfeeding revealed significant differences in active RAI and snore arousal index during active, but not when these variables were corrected by maternal education and parental smoking using ANCOVA. 


\subsection{Introduction}

\subsection{Preamble}

Sleep or sleep-like states have been observed not only in humans, but also in nearly all mammals, birds, reptiles, fish, and other animals investigated. Indeed, even organisms thought to be evolutionally distant from humans such as the fly genus Drosophila and the flat worm Caenorhabditis elegans (C. elegans) demonstrate sleep from a behavioral perspective (Allada \& Siegel, 2008). Furthermore, both rats and flies systematically deprived of sleep will die (Allada \& Siegel, 2008; Rechtschaffen, Gilliland, Bergmann, \& Winter, 1983; Shaw, Tononi, Greenspan, \& Robinson, 2002). This evidence, put together, suggests that sleep provides some function critical to sustaining life while at the same time implying that sleep in humans may have evolved from the simpler sleep found in other organisms. I proposed for the purposes of this dissertation that sleep is not only required for the preservation of life but also assists in the neural development of the organism itself, as others have posited (Marks, Shaffery, Oksenberg, Speciale, \& Roffwarg, 1995; Roffwarg, Muzio, \& Dement, 1966). Specifically, I hypothesized that global white matter development was contingent upon consolidated, high-quality REM sleep undisturbed by respiratory anomaly that is sometimes observed in the pediatric population (sleep disordered breathing, SDB).

The following chapter will first outline the characteristics of normal sleep, and discuss how disordered sleep differs from the normal sleep, how it can develop, and its potential effects on the brain. Further, this chapter will articulate potential ways to prevent disordered sleep, possibly ameliorating negative effects on brain development. Finally, the actual dissertation and aims of the study will be stated. 


\subsection{Normative Sleep}

In order to appropriately characterize disordered sleep, we must first define normative sleep. Though much of the sleep research that occurred in the early $20^{\text {th }}$ century involved direct observation and anecdotal reports of dreaming, technological developments since the 1950s have allowed us to more precisely monitor the brain. These techniques, which have now become standard, include electroencephalography (EEG), electrooculography (EOG), and electromyography (EMG). The electrical recording produced by EEG, EOG, and EMG (which partially makes up the modern approach of polysomnography (PSG)) can be quite distinctive, allowing for sleep staging across the night for the individual participant undergoing these techniques. From these developments, the American Academy of Sleep Medicine (AASM) has established standardization for how these methods are performed as well as guidelines for sleep staging and what is considered "normal" for various age groups (Berry, Brooks, et al., 2015).

\subsubsection{Infant Sleep}

Infant sleep is perhaps the most difficult to characterize, if only because of the challenge presented in completing a full PSG montage on a young infant as well as the rapid changes and indeterminate sleep characteristics present in this age group. For instance, young infants are generally not classified by the standard sleep stages in adults such as wake, N1, N2, N3, and REM, but are rather reduced to Active (considered similar to REM), Quiet (considered similar to NREM), and Indeterminate (Anders, Emde, \& Parmelee, 1971; Schade, Bauer, Warren, \& 
Montgomery-Downs, 2016). Sleep duration, circadian rhythms, and EEG characteristics are still quite variable during this time, as they are not fully developed, which adds to the difficulty of sleep staging. Nevertheless, it was discovered early in modern sleep research that, of the total amount of time engaged in sleep, the proportion of REM sleep (or Active sleep) was by far the greatest in the first few days of life ( $\sim 50 \%)$ and diminished rapidly to near-adult proportions by 2 to 3 years old (Roffwarg et al., 1966). This observation has led to speculation that REM sleep may have an important role in brain development and maturation early in life. This hypothesis will be further explored in this dissertation.

\subsubsection{Pediatric Sleep}

Even by early childhood sleep continuity and EEG patterns are much more characteristic than during early infancy, though these patterns are still developing (Berry, Brooks, et al., 2015; Schade et al., 2016). By 6 months of age the active, quiet, and indeterminate stages have usually been replaced with the standard stages used in adults (Anders et al., 1971; Berry, Brooks, et al., 2015). Young children have a similar amount of each sleep stage as adults, with slightly more N3 sleep (Biggs et al., 2012; Mazzotti et al., 2014; Schade et al., 2016). The N3 stage is generally associated with the release of various growth factors and human growth hormone, and is thought to be related to physical regeneration (Schade et al., 2016; Takahashi, Kipnis, \& Daughaday, 1968) . In addition, the presence of high amplitude, slow frequency delta waves is characteristic of N3 sleep, which is considered the deepest stage of sleep (Biggs et al., 2012; Chu, Leahy, Pathmanathan, Kramer, \& Cash, 2014; Schade et al., 2016). The increased 
amount of N3 sleep in young children relative to adults may therefore be logical in that body growth and development is more critical during this early life stage.

Sleep duration, EEG characteristics, and sleep staging proportions continue to develop and change through school-aged children and adolescents, but these changes are much more modest than earlier in development (Schade et al., 2016).

\subsubsection{Adult Sleep}

Sleep EEG characteristics are relatively consistent in early and middle adulthood and standardly scored in healthy individuals (Berry, Brooks, et al., 2015). Healthy adult sleep is divided into two broad categories; non-rapid eye movement sleep (NREM) sleep and rapid eye movement (REM) sleep. The NREM sleep is further subdivided into N1 (lightest stage), N2 (nextlightest stage; majority of sleep), and N3 (deepest stage; Carskadon \& Dement, 2011). From the perspective of EEG, N1 can be visualized as relatively low-amplitude, higher frequency (4-7 hz) theta waves which dominant this stage (Castronovo \& Butkov, 2007). Stage N2 is a deeper stage of sleep than N1, and can be identified by large inflections in the EEG signal known as Kcomplexes, as well as short bursts of increased frequency lasting at least 0.5 second known as sleep spindles (Carskadon \& Dement, 2011; Castronovo \& Butkov, 2007). The presence of Kcomplexes or sleep spindles in the EEG is a scoring standard and generally confirms that a sleeper is in stage N2 (Berry, Brooks, et al., 2015). The final stage of NREM sleep, N3, is the easiest to identify. Stage N3 consists of very low frequency delta waves $(0.5-2 \mathrm{hz})$ coupled with large amplitudes (Carskadon \& Dement, 2011; Castronovo \& Butkov, 2007). This stage is most closely associated with physical regeneration as human growth hormone $(\mathrm{HGH})$ and other 
growth factors are released, resulting in tissue repair and accelerated immune function (Schade et al., 2016; Takahashi et al., 1968).

REM sleep is a stage of sleep very different from the NREM stages. During REM sleep, an individual is typically completely paralyzed with no muscle tone. In addition, REM sleep is probably most famous for dreaming; during this stage the most salient and memorable dreaming occurs (Berrien, 1930).

By proportion, about $5-10 \%$ of total adult sleep is spend in N1, $45-55 \%$ in $\mathrm{N} 2,15-20 \%$ in N3, and 20-25\% in REM sleep (Carskadon \& Dement, 2011; Mazzotti et al., 2014; Ohayon, Carskadon, Guilleminault, \& Vitiello, 2004). Note that the total amount of REM sleep in adults is significantly lower than it was in early infancy ( $50 \%)$. In addition, healthy adult sleep is consolidated into these changes with few nocturnal awakenings or interruptions. The adult sleep cycle is roughly 90 minutes on average and progresses through each of the stages at least once (Carskadon \& Dement, 2011; Castronovo \& Butkov, 2007). These cycles tend to contain more NREM sleep (specifically N3 sleep) earlier in the night, while towards the end of the night REM sleep becomes more prevalent (Carskadon \& Dement, 2011; Castronovo \& Butkov, 2007). In the healthy individual no respiratory problems, including snoring, are present during sleep.

\subsection{Disordered Sleep}

Sleep disorders are unfortunately very common in the United States for all age groups, with general sleep disturbance affecting $85 \%$ of individuals over 1 year old (Roth \& Roehrs, 2003; Sarris \& Byrne, 2011) and an estimated $10-30 \%$ of 6-month old infants were reported to have behavioral sleep disorders by parents (Blunden, 2012; Byars, Yolton, Rausch, Lanphear, \& 
Beebe, 2011; Morgenthaler et al., 2006). Many of the same disorders which can affect adults also affect younger age groups as well, but the manifestation of these disorders can vary. Sleep disorders which have been well studied include, but are not limited to, insomnias (Roth \& Roehrs, 2003; Sarris \& Byrne, 2011), narcolepsy (Buttoo, Pandi-Perumal, \& Guilleminault, 2016; Goldbart et al., 2014), parasomnias (Owens \& Mohan, 2016; Redington, Mattice, \& Eckhardt, 2007), sleep-related movement disorders (Comella, 2014; Manconi et al., 2012; Sotelo, 2007), central sleep apnea, (Aurora et al., 2012; Javaheri, 2010; Linley, Papadima, \& Fabiani, 2007) and sleep-disordered breathing (Lumeng \& Chervin, 2008; Montgomery-Downs, Crabtree, Capdevila, \& Gozal, 2007; Redline et al., 2004; Short \& Banks, 2014). This section will briefly cover the first five, with a heavy emphasis on sleep-disordered breathing.

\subsubsection{Overview of Sleep Disorders}

Chronic insomnia has an estimated prevalence of $10 \%$ in the adult population (Pigeon, 2010; Roth \& Roehrs, 2003; Simon \& Vonkorff, 1997). Characterized by difficulty initiating and/or maintaining sleep, insomnia can have adverse behavioral daytime consequences such as severe sleepiness, memory impairment, and a more frequent number of accidents (Roth \& Roehrs, 2003). In addition, insomnia can affect any age group. Transient insomnia typically will last only a week or two, while chronic insomnia will range from months to years (Roth \& Roehrs, 2003).

When insomnia occurs by itself it is referred to as primary insomnia, however much more common is secondary insomnia in which insomnia is actually comorbid with a myriad of other disorders (Pigeon, 2010; Simon \& Vonkorff, 1997). Insomnia appears to be comorbid 
most frequently with psychiatric disorders such as depression and mood disorders, but also with other sleep disorders such as restless leg syndrome (RLS) and periodic leg movement disorder (PLMD; Pigeon, 2010; Roth \& Roehrs, 2003; Simon \& Vonkorff, 1997). Consequences associated with insomnias are complicated by these comorbidities, but in general transient insomnia results in loss of attention and daytime sleepiness, while chronic insomnia is associated with decreased quality of life, decreased productivity, and functional impairment (Roth \& Roehrs, 2003; Simon \& Vonkorff, 1997). However, exact effects of insomnia will depend on other disorders in cases of secondary insomnia (Pigeon, 2010; Roth \& Roehrs, 2003).

Narcolepsy with cataplexy, also called narcolepsy type 1, affects about 1 out of every 4,000 people in the general population (Buttoo et al., 2016; Goldbart et al., 2014). Individuals with narcolepsy suffer from excessive daytime sleepiness (EDS), but also cataplexy (sudden muscle weakness during wake often evoked by strong emotions), sleep paralysis during wake, and hypnogogic hallucinations (Buttoo et al., 2016). The etiology of type 1 narcolepsy stems from the central nervous system's inability to regulate the sleep/wake cycle. One major current hypothesis about the cause of narcolepsy, known as the autoimmune hypothesis, has been proposed due to the strong association in type 1 narcolepsy with mutations in the human leukocyte antigen (HLA) gene complex which encodes for cell-surface receptor proteins used in immune function (Giacomini, Tognoli, \& Hydes, 2007; Goldbart et al., 2014; Mignot et al., 2001). Furthermore, the disorder is almost always associated with a loss of orexin (hypocretin)producing cells in the hypothalamus (Buttoo et al., 2016; Goldbart et al., 2014), leading to the idea that an autoimmune response may target these cell populations. Aside from EDS and other major symptoms of narcolespy, individuals may also suffer deficits in attention and memory, 
experience increased pain, and suffer from depression or anxiety disorders (Buttoo et al., 2016). Treatment for narcolespy generally relies on multiple pharmaceutical agents, including medications to treat EDS, cataplexy, and promote sleep consolidation (Giacomini et al., 2007). Good sleep hygiene and behavioral therapy may be used to supplement pharmacological treatments (Giacomini et al., 2007).

Parasomnias are a classification of sleep disorders that are defined as undesirable physical events or phenomena that occur during sleep, primarily during NREM sleep stage 3 and REM sleep (Owens \& Mohan, 2016; Redington et al., 2007). These disorders are varied, but can broadly be narrowed into disorders of arousal, REM sleep disorders and other (enuresis, exploding head syndrome, sleep-related dissociative disorders, etc.; Redington et al., 2007). Furthermore, arousal parasomnias, REM parasomnias, and other parasomnias are all most prevalent during childhood and wane significantly with age. Nonetheless, they are present in all ages.

Although not all parasomnias will be discussed here, some of the more common types include confusional arousals (arousal-type, NREM), sleep terrors (arousal-type, NREM), sleepwalking (arousal-type, NREM), REM sleep behavioral disorder (REM-type), and sleep paralysis (REM-type). Other parasomnias not discussed here include enuresis (bedwetting), exploding head syndrome (arousal from imagined loud noise or "explosion" in the head during the wake/sleep transition), nightmares (frightening dreams during REM), sleep-related eating disorder (eating during sleep; unknown to the individual), sleep-related dissociative disorders (behaviors throughout the sleep period that can include violence, screaming, running, etc.), and 
sleep-related hallucinations, among others (hypnagogic and hypnopompic hallucinations; Redington et al., 2007).

Confusional arousals are most common during NREM stage 3 earlier in the night with the individual exhibiting disorientation, unclear thoughts, agitation, and slowed speech upon arousal (Owens \& Mohan, 2016; Redington et al., 2007). Individual episodes can last between 5 to 60 minutes, and the episodes may persist for 6-12 months (Owens \& Mohan, 2016;

Redington et al., 2007). Etiology could include forced awakening from deep sleep, sleep deprivation, or neurological disorders (Redington et al., 2007).

Sleep terrors occur suddenly and acutely during NREM stage 3 sleep and are characterized by dramatic loud screaming, crying, lack of responsiveness to others, and the appearance of panic (Owens \& Mohan, 2016; Redington et al., 2007). Although fairly common in children, they typically last only 5 minutes maximum. Interestingly, although inconsolable during the sleep terror, children will typically have no memory of the event (Redington et al., 2007).

Sleepwalking, also known as somnambulism, is ambulatory motor behavior and sometimes associated purposeless or purposeful tasks during NREM stage 3 sleep (Owens \& Mohan, 2016; Redington et al., 2007). Sleepwalking is much more likely to occur earlier in the night. Sleepwalking severity can range from mild (barely getting out of bed) to performance of bizarre and occasionally destructive tasks, such as driving (Owens \& Mohan, 2016; Redington et al., 2007). The affected individual will have little to no recollection of the event or events that transpired during sleepwalking. Attempts to communicate with a sleepwalking individual are generally futile, with duration of episodes ranging from several minutes to an hour (Owens \& 
Mohan, 2016; Redington et al., 2007). Sleepwalking is much more common in children than in adults.

REM sleep behavioral disorder is a sleep disorder which involves temporary lack of muscle atonia during REM sleep, resulting in motor activity (Nevsimalova, Prihodova, Kemlink, \& Skibova, 2013; Redington et al., 2007; Stores, 2008). This could be most appropriately thought of as "acting out one's dream". These behaviors, as in sleepwalking, can range from simple to complex, and can result in serious injury. However, unlike in sleepwalking, a patient suffering from REM sleep behavioral disorder will often have their eyes closed as opposed to wide open (Redington et al., 2007). This disorder is more common during the second half of the night, and episodes will cease with sudden alertness and awakening.

Sleep paralysis is a condition in which the affected cannot move either during sleep onset or transition from sleep to wake (Redington et al., 2007). It is usually only voluntary movements which are paralyzed, with respiratory muscles spared (Nevsimalova et al., 2013; Redington et al., 2007; Stores, 2008). The episode may last between several seconds to minutes. Consciousness is maintained throughout the episode, making the experience very frightening and unpleasant to affected individuals (Redington et al., 2007).

Moving on from parasomnias, the next class of sleep disorders discussed are the sleeprelated movement disorders. Primarily, this includes both RLS and PLMS. Other disorders that fall under this category include sleep-related leg cramps, sleep-related bruxism (teeth grinding) and others but will not be discussed here.

RLS is not actually a sleep disorder despite its ability to manifest at night but a wake disorder (Comella, 2014; Manconi et al., 2012; Sotelo, 2007). Characterized by unpleasant and 
abnormal sensations in the legs at rest, RLS can often have a diurnal pattern becoming significantly worse at nighttime and preventing an individual from transitioning from wake to sleep. It is classified as a sleep-related movement disorder due to the impact RLS can have on sleep and daytime functioning. Individuals with RLS can complain of insomnia or excessive daytime sleepiness (EDS).

RLS prevalence in the general population has been reported to be anywhere between $2 \%$ to $24 \%$ (Comella, 2014; Sotelo, 2007), making it difficult to exactly determine how many people are affected. Although seen in all ages, it is more common in the elderly and twice as likely in women (Comella, 2014; Sotelo, 2007). Familial history is present in $50-60 \%$ of RLS sufferers (Comella, 2014; Sotelo, 2007). RLS is also often seen in connection with iron deficiency and iron deficiency conditions, as well as use of dopamine-receptor antagonists (Comella, 2014; Sotelo, 2007). Treatment of RLS includes both pharmacological and non-pharmacological interventions, with sleep hygiene coaching, progressive leg exercise, and even placebo utilized in the case of the latter (Comella, 2014; Sotelo, 2007). Dopaminergic agonists, especially those that target the D3 receptor, are considered to be the frontline treatment options (Comella, 2014; Manconi et al., 2012; Sotelo, 2007). Other treatment options include other opioids or benzodiazepines, or even iron supplementation in the case of low iron levels (Sotelo, 2007). Periodic leg movements of sleep (PLMS) are repetitive, rhythmic movements and jerks of the lower limbs during sleep that occur every 5 to 90 seconds and in a string of at least 4 movements (Becker \& Forester, 2007; Haba-Rubio et al., 2016; Manconi et al., 2012). Although the clinical relevance of PLMS as a primary symptom is disputed (Becker \& Forester, 2007; Manconi et al., 2012), they are seen in $80-90 \%$ of individuals with RLS (Becker \& Forester, 2007; 
Comella, 2014; Manconi et al., 2012). A common etiology between RLS and PLMS may exist, but is controversial (Manconi et al., 2012).

Prevalence of PLMS is fairly high with one study indicating $28.6 \%$ of the adult general population suffer from PLMS at least 15 times per hour (Haba-Rubio et al., 2016). It seems to be less common in 5-17 year old children, with only $1.5 \%$ meeting the same criterion (Marcus et al., 2014). It should be noted that although a diagnosis of PLMS, which can only be diagnosed with PSG, is suggestive of RLS, the presence of PLMS alone is not sufficient (Becker \& Forester, 2007). RLS can only be diagnosed through patient interview (Becker \& Forester, 2007).

Although PLMS may occur with or without cortical and autonomic arousals (Becker \& Forester, 2007; Manconi et al., 2012), recent research suggests PLMS may be the symptom of these arousals and not the cause (Manconi et al., 2012). If true, treatment of PLMS alone may have limited utility (Manconi et al., 2012). Nevertheless, PLMS is typically treated with dopaminergic agents, similar to that of RLS (Becker \& Forester, 2007; Manconi et al., 2012).

Central sleep apnea (CSA) is neurological condition in which the central nervous systems fails to start or maintain the action of respiratory muscles to make breathing possible (Aurora et al., 2012; Javaheri, 2010; Linley et al., 2007). During a CSA event, specific neurons in the pons and the medulla responsible for breathing fail to stimulate respiratory effort (Aurora et al., 2012; Javaheri, 2010; Linley et al., 2007). This is in stark contrast to SDB and OSA, where respiratory effort is present, but there is a lack of respiration due to a blockage or obstruction within the airway. By definition, the CSA event must result in at least 10 seconds of no breathing and no effort for it to be classified as an apnea. 
Clinically, CSA only constitutes about 5-10\% of all apnea cases (Linley et al., 2007). In full disclosure, CSA is not a singular disorder but actually has numerous specific causes (Aurora et al., 2012; Javaheri, 2010; Linley et al., 2007). Unfortunately, CSA has been strongly linked with systolic heart failure (Aurora et al., 2012; Javaheri, 2010; Linley et al., 2007) with an estimated $31 \%$ of individuals with systolic heart failure worldwide also suffering from CSA (Javaheri, 2010). Treatments for CSA is limited, but some promising options include continuous positive airway pressure (CPAP; Aurora et al., 2012; Linley et al., 2007), adaptive servo-ventilation (also a positive airway pressure device, but adaptive; Aurora et al., 2012), and to some extent certain medications (Aurora et al., 2012; Linley et al., 2007). Positive airway pressure devices are generally considered to be the standard treatment for CSA, which uses pressurized air to maintain airway width and ensure continued respiration (Aurora et al., 2012; Linley et al., 2007).

Sleep-disordered breathing (SDB) is a serious condition which, as the name implies, disrupts breathing during sleep and results in chronic and frequent intermittent hypoxias, some of which can last in the tens of seconds (Kohler et al., 2009; Lumeng \& Chervin, 2008; Montgomery-Downs et al., 2007; Redline et al., 2004; Short \& Banks, 2014). SDB is a spectrum disorder, and can range from mild snoring to severe obstructive sleep apnea. Individuals are, unfortunately, often unaware of their own affliction (arousals from hypoxia do not reach level of consciousness), and requires another person to notice really severe snoring or witness apneas and recommend treatment. Furthermore, the chronic intermittent hypoxias not only cause needless physical stress on the body, but also significantly fragments sleep (Daurat, Foret, Bret-Dibat, Fureix, \& Tiberge, 2008; Gozal \& Pope Jr., 2001; Kohler et al., 2009; Lumeng \& 
Chervin, 2008). Sleep fragmentation, specifically in the context of the respiratory arousals from SDB, is often comprised of small continuous chunks of sleep separated by numerous brief respiratory arousals. Therefore, sleep fragmentation represents non-consolidated, highly interrupted sleep which can be quantified using AHI (defined below), along with other metrics. Finally sleep fragmentation can vary based on the stage of sleep; sleep fragmentation need not be, and very rarely is, uniform across the night.

SDB is caused by soft tissue collapse within the airway, causing an obstruction which interferes with respiration during sleep. The precise soft tissue area which causes the obstruction can vary by age group; in children it is often cause by enlarged tonsils or adenoids (Kohler et al., 2009), while adults tend to suffer from blockages that originate at the back of the tongue or nasal passage. These blockages cause the primary symptoms of SDB; they are the source of the apneas and hypopneas and often can produce loud snoring. This snoring, if significantly severe, could be loud enough to force a sleeping partner out of the room or possibly resonate the entire house (Ayalon, Liu, \& Ancoli-Israel, 2004).

From a quantitative perspective SDB severity is generally measured using the widely cited apnea-hypopnea index (AHI; Berry et al., 2015). AHI is calculated by counting the number of apneas (which consist of complete stoppage of breathing) and hypopneas (under-breathing, or partial breathing) that occur per hour over the course of a sleeping period. Therefore, the higher the $\mathrm{AHI}$ the more severe the SDB. Also, in order to be considered a clinical event that is included in $\mathrm{AHI}$ calculation, the respiratory incident must last a minimum of 10 seconds and be associated with a 3-4\% oxygen desaturation. Finally, it should be noted that SDB is often 
associated with obstructive apneas (where there is measured respiratory effort, but little or no actual airflow through the esophagus) more than any other apneas.

Physiological consequences of SDB that occur in all age groups include excessive sleep fragmentation, chronic oxygen desaturation, and possible alterations to sleep architecture (Daurat et al., 2008; Gislason \& Benediktsdóttir, 1995; Gozal \& Pope Jr., 2001; Lumeng \& Chervin, 2008; Redline et al., 2004; Short \& Banks, 2014). Extreme fatigue and sleepiness during the day can also result (Ayalon et al., 2004). Many other serious consequences also exist, but are generally age-specific, and thus will be discussed in the upcoming sections.

\subsubsection{Sleep-Disordered Breathing in Adults}

Moderate to severe SDB is thought to affect $10 \%$ of men $30-49$ years of age, and $17 \%$ of men $50-70$ years of age (it is noticeably less in women; $3 \%$ and $9 \%$ respectively; Peppard et al., 2013). Aside from the traditional health consequences of daytime sleepiness, fatigue, irritability, sleep fragmentation and chronic deoxygenation during sleep (Ayalon et al., 2004; Beebe \& Gozal, 2002; Grigg-Damberger \& Ralls, 2012; Peppard et al., 2013), the major longterm risk for adults is often cardiovascular (Arzt, Young, Finn, Skatrud, \& Bradley, 2005; Ayalon et al., 2004; Nieto et al., 2000). SDB has in particular been linked to increased risk of hypertension and increased risk for stroke (Arzt et al., 2005; Nieto et al., 2000). As such, adults with SDB have a higher risk of premature death (Ellen et al., 2006; Weaver et al., 2012) and individuals with sleep apnea are more likely to be involved in motor vehicle accidents (Ellen et al., 2006). Due to the potentially vast array of health maladies associated with SDB, diagnosis and treatment is essential. 
The best course of treatment for SDB varies based on the age of the individual. In adults, the treatment of choice is continuous positive airway pressure (CPAP), which has been demonstrated to be fairly effective overall (Weaver et al., 2012). Continuous positive airway pressure works by providing continuous pressure within the airway such that it prevents the collapse of soft tissues. The goal of CPAP, of course, is to provide sufficient pressure during all phases of respiration to prevent obstruction and the cessation of breathing. Although other treatments are available, such as pharmaceuticals and oral-nasal devices, CPAP remains the most common and arguably most effective treatment available to adults (Ayalon et al., 2004; Weaver et al., 2012).

\subsubsection{Sleep-Disordered Breathing in Children}

Childhood, for the purposes of this section, refers to any individual between the ages of 2-18 years old, unless otherwise indicated. Approximately $1-4 \%$ of children of all ages suffer from OSA, the most severe form of SDB, according to conservative estimates (Lumeng \& Chervin, 2008; Schade et al., 2016), although the entire range of SDB has been associated with deficits (Bourke et al., 2011a, 2011b; Grigg-Damberger \& Ralls, 2012; Perfect, Archbold, Goodwin, Levine-Donnerstein, \& Quan, 2013). Furthermore, there is reason to believe that these estimates may be too low as $10-20 \%$ of all children under the age of 7 may suffer from "habitual snoring", the primary symptom of SDB, as indicated by maternal report (Bonuck et al., 2011; Lumeng \& Chervin, 2008). These data suggest that SDB may be significantly underdiagnosed, at least among younger children. This is of some concern as SDB during childhood, a sensitive phase of development as a whole, has been linked to decreases in 
neurocognitive outcomes (Bourke et al., 2011a; Halbower et al., 2006; Karpinski, Scullin, \& Montgomery-Downs, 2008; Kohler et al., 2009), school performance (Bourke et al., 2011a; Gozal \& Pope Jr., 2001), increases in behavioral problems (such as attention difficulties, adaptive functioning, and social functioning [Bourke et al., 2011b; Chervin et al., 2006; Jackman et al., 2012; Perfect et al., 2013]), as well as other negative outcomes (Chervin et al., 2006). More specifically, some of the documented negative neurocognitive effects include impaired executive function (Bourke et al., 2011a; Halbower et al., 2006; Karpinski et al., 2008; Kohler et al., 2009), inhibition control (Karpinski et al., 2008; Kohler et al., 2009), working memory (Halbower et al., 2006; Karpinski et al., 2008; Kohler et al., 2009), mental flexibility (Bourke et al., 2011a), and strategic planning (Bourke et al., 2011a; Karpinski et al., 2008; Kohler et al., 2009). Although commonly reported in diagnosed childhood SDB, even habitual snoring alone has been linked to daytime sleepiness in school-aged children (Chervin et al., 2002). Behaviorally, the presence of SDB is correlated with ADHD-like symptoms such as hyperactivity and inattention (Chervin et al., 2002; Jan, Yang, \& Huang, 2011; Perfect et al., 2013), and although not exclusive to SDB, it has been reported in one study that $83.3 \%$ of ASD individuals had sleep-related complaints by survey (Ming, Sun, Nachajon, Brimacombe, \& Walters, 2009). This result was partially supported by PSG (Ming et al., 2009). Furthermore, it has been found that in children with ASD there is a higher prevalence of SDB (Ming et al., 2009), and ASDaffected individuals often suffer snoring, apneas, and sleep fragmentation (Ming et al., 2009; Tsai et al., 2012). Finally, and perhaps most sobering of all, is that the developmental effects conferred upon children with SDB may be irreversible in some cases (Bonuck \& Grant, 2012; Bourke et al., 2011a; Kohler et al., 2009). Even after 6 months of adenotonsillectomy treatment, 
it was reported that 3-12 year old children who had SDB had a 10 point reduction in IQ compared to age-matched controls (Kohler et al., 2009; Schade et al., 2016).

On a lighter note, childhood SDB can usually be treated. Like in adults, both CPAP and orthodontic appliances are reasonable methods for SDB correction (Guilleminault, Lee, \& Chan, 2005). However, the most common treatment for pediatric OSA and SDB is actually adenotonsillectomy (Guilleminault et al., 2005; Kohler et al., 2009). This treatment option involves surgical removal of the adenoids and/or tonsil tissues which can often become swollen or enlarged in children and block the airway during sleep. Research studies focused on treatment efficacy have suggested that adenotonsillectomy is reasonably effective and can lead to improvements in cognitive, academic, and behavioral outcomes (Friedman et al., 2003; Marcus et al., 2013; Schade et al., 2016), although this is not always the case (Kohler et al., 2009; Marcus et al., 2013). Orthodontic equipment could also be effective for children, but its use is relatively uncommon and research in this area is sparse (Guilleminault et al., 2005). Continuous positive airway pressure, which is very effective for adults, is less so for children mainly due to compliance issues and parental training (Archbold, 2013; Archbold \& Parthasarathy, 2009). Nevertheless, with the correct training program and approach this method can be effective (Archbold, 2013; Archbold \& Parthasarathy, 2009).

Although adenotonsillectomy and other treatments have been effective in managing SDB, the ideal scenario would involve mastering SDB prophylactics. One preventative measure recently proposed to combat SDB is breastfeeding (Montgomery-Downs et al., 2007). Although not a treatment, results from this study of 4-8 year old children demonstrated that the greater the duration of breastfeeding as an infant, the greater the reduction in SDB at a later age by 
correlation (Montgomery-Downs et al., 2007). This study quantified SDB using AHI values, while gathering infant feeding information by parental report. Although subsequent research in this area is definitely needed, this study shows some promise in preventing SDB altogether earlier, thus preventing the need for later, invasive treatments during childhood.

\subsubsection{Sleep-Disordered Breathing in Infants}

Children are susceptible to the negative consequences of SDB in numerous ways, but infants are likely even more so. Although infants are rarely diagnosed with SDB due to the inherent difficulty in ordering sleep tests for this age group, it is estimated that between 12$20 \%$ of infants snore habitually, which is the primary symptom of SDB (Byars et al., 2011; Schade et al., 2016). Even among infants with mild SDB it has been shown that snoring through the majority of the first year of life is correlated to decreased scores on developmental instruments compared to controls (Grigg-Damberger \& Ralls, 2012; Piteo et al., 2011; Schade et al., 2016). In addition, $2-4 \%$ of infants are suspected to suffer from OSA (Robison, Wilson, Otteson, Chakravorty, \& Mehta, 2013; Schade et al., 2016). This same study indicated that OSA even in infancy could be associated with behavioral problems immediately (Robison et al., 2013; Schade et al., 2016). It is therefore essential to have any SDB-spectrum disorder treated as soon as possible, not only to mitigate negative consequences associated with infancy but also to prevent the childhood deficits which are more thoroughly documented and potentially difficult to reverse (Barnes et al., 2009; Key, Molfese, Brien, \& Gozal, 2009; MontgomeryDowns, Jones, Molfese, \& Gozal, 2003; Schade et al., 2016). 
Since infants are rarely tested for SDB using polysomnography, the gold standard for

diagnostic testing (Marcus et al., 2012; Tan, Gozal, Ramirez, Bandla, \& Kheirandish-Gozal, 2014), it is logical to conclude that infants are also rarely treated. Despite the lack of SDB treatment for this age group, recent evidence has suggested there is a preventative measure that mothers can employ (Brew et al., 2014; Montgomery-Downs et al., 2007). A greater duration of breastfeeding throughout infancy, as opposed to formula-feeding, has been shown to be significantly correlated with lower AHI values (Montgomery-Downs et al., 2007) and reduced risk of habitual snoring (Brew et al., 2014) in children. Although evidence in this area is still somewhat preliminary, this may suggest that infant feeding methods might have a later effect on SDB severity in those afflicted, or lower the probability of disease development entirely. The precise mechanism for how this might occur will be discussed in the upcoming sections.

\subsection{Infant Feeding Methods}

Mothers of young infants are generally presented with a continuum of 3 choices as to how to feed their child initially; exclusive breastfeeding, mixture of breastfeeding and formulafeeding, or exclusive formula-feeding. Certainly these methods might change as the child grows before being weaned to solid foods, but at least for the first few months mothers often develop a set pattern of feeding (Kent et al., 2013). Breastfeeding, and sometimes exclusive breastfeeding, is often associated with a wide range of beneficial effects (J. W. Anderson, Johnstone, \& Remley, 1999; Brew et al., 2014; Deoni et al., 2013; Galbally, Lewis, McEgan, Scalzo, \& Islam, 2013; Isaacs et al., 2008; Isaacs, Fischl, Quinn, Chong, \& Gadian, 2010; Kafouri et al., 2013; Kramer et al., 2008; McCrory \& Murray, 2013; Mindell, Du Mond, Tanenbaum, \& 
Gunn, 2012; Montgomery-Downs et al., 2007; Mortensen, Michaelsen, Sanders, \& Reinisch, 2002). There are some who are critical of the breastfeeding literature, specifically regarding its causal contribution to any beneficial outcomes observed because of the many confounding variables related to breastfeeding that are present (A. Anderson \& Burggren, 2014), particularly the effect of socioeconomic status. Therefore, the recommendation that mothers should breastfeed their infants remains controversial. In the following sub-chapter, infant feeding methods and potential effects on sleep, brain development, and behavioral and academic outcomes will be discussed. In particular, the effects breastfeeding might have on SDB will emphasized as this relationship will be central to my proposed dissertation.

\subsubsection{Infant Feeding Methods and Sleep}

The studies which have investigated effects of breastfeeding on the developing infant's general sleep quality have generally been equivocal. On one hand, there is evidence to suggest that breastmilk may have larger quantities of melatonin than formula milk (Engler, Hadash, Shehadeh, \& Pillar, 2012; Schade et al., 2016). Melatonin is not only known to induce sleepiness during the night, but also to assist in circadian regulation. Therefore, lower levels of melatonin in formula milk may result in overall less consolidated sleep and less developed circadian rhythms than their breastfed peers, though this would need to be supported by additional scientific studies. Also, infant breastfeeding has been linked to a reduction in infantile colic (Engler et al., 2012; Schade et al., 2016).

On the other hand, infant breastfeeding is associated with negative aspects of sleep as well. Infants who were breastfed may have more difficulty sleeping by themselves (Galbally et 
al., 2013) and have less consolidated sleep with an overall increase in sleep fragmentation from close proximity to the mother (Galbally et al., 2013; Mindell et al., 2012; Schade et al., 2016). Self-report surveys also have indicated that breastfed infants may have more nocturnal awakenings than non-breastfed infants, and that these awakening may also be longer (Engler et al., 2012; Galbally et al., 2013; Mindell et al., 2012; Schade et al., 2016), although this may be because movement from the parent is waking the child rather than any intrinsic properties of breastfeeding (Ramamurthy et al., 2012; Schade et al., 2016). Indeed, by the age of 9 months, differences in awakenings between breastfed and formula-fed infants are no longer present (Mindell et al., 2012; Schade et al., 2016). Therefore, it may be that although some negative sleep consequences exist, they could be short lived. Despite this, there are other observed effects of breastfeeding that may imply a positive long-term developmental impact.

\subsubsection{Breastfeeding and Sleep-Disordered Breathing}

Although the link between breastfeeding and SDB is still, relatively speaking, in its infancy, there is growing evidence that this type of feeding can have a protective effect from snoring and breathing problems during sleep (Brew et al., 2014; Galbally et al., 2013;

Montgomery-Downs et al., 2007), both immediately (Galbally et al., 2013) and later in life (Brew et al., 2014; Montgomery-Downs et al., 2007). In a population of habitually-snoring children, it was discovered that not only was breastfeeding exposure predictive of decreased SDB severity, but that there was a dose-dependent effect in which the duration of breastfeeding was correlated with reduced disease measured by AHI (Montgomery-Downs et al., 2007). Although it is not yet known how infant feeding methods could have protective effects against SDB 
mechanistically there are several hypotheses. Regardless, the few studies which have investigated this relationship seem to indicate that breastfeeding might be able to reduce the severity of SDB in affected children, if not prevent disease development altogether.

\subsubsection{Breastfeeding and Brain Development}

Many of the studies which focus on the link between breastfeeding and brain development progress by either looking at the brain indirectly using a neuroimaging methodology and asking about breastfeeding information retrospectively, or by collecting neurocognitive information such as IQ to infer brain function from this information. Since the latter will be discussed in the next sub-section, this section will focus on the neuroimaging research between feeding methods and brain development. Generally speaking, MRI is the candidate of choice for these types of studies due to its excellent spatial resolution and deep brain imaging, lack of radioactive exposure in children, and general lack of wires and extensive setup.

MRI studies that have investigated this link usually have focused on white matter (Deoni et al., 2013; Isaacs et al., 2008, 2010), although not always (Kafouri et al., 2013). The white matter in the brain is representative of axonal connections between neurons and is generally quantified either through volumetric analysis throughout the brain in various regions or by using diffusion tensor imaging (DTI). The DTI analyses used are thought to be indicative of axonal quality and degree of connectivity between regions; thus a higher DTI score would be viewed more favorably. The reason for this is relatively simple: DTI is a measure primarily of the diffusion of water molecules in particular regions of the brain. Since water inside white matter 
axons should be constrained by the walls of these axons, a higher DTI value would imply that less water is flowing perpendicular to the axon and therefore that axon may be more heavily myelinated or lack structural damage. In contrast, a lower DTI value in the white matter implies either a poorly myelinated axons in the region or structural damage to those axons (Soares, Marques, Alves, \& Sousa, 2013; Wozniak \& Lim, 2006). Although DTI is becoming more and more commonly used to assess white matter structure over volumetric analysis, white matter volumes are still heavily relied upon (Frazier, Keshavan, Minshew, \& Hardan, 2012; Hardan et al., 2009; Haut et al., 2006; Paus et al., 2008; Shashi et al., 2012; Toplak, West, \& Stanovich, 2013).

In breastfeeding research in particular, an increased consumption of breastmilk (reported as percentage of expressed breastmilk in diet) is positively correlated with total white matter volume in both the right and left hemispheres in adolescence, especially in males (Isaacs et al., 2010). A different study had found that breastfeeding duration was positively correlated with a measure of white matter microstructure, thought to be indicative of greater white matter maturity (Deoni et al., 2013). In that study, the myelin water fraction (fraction of water bound to myelin in a particular voxel) was used to assess white matter microstructure quality. Other studies have focused more on breastfeeding and grey matter, one of which found that a high nutrient diet early in life was correlated with caudate volume size, a subcortical greymatter structure, compared to adolescents who had a low nutrient diet early in life (Isaacs et al., 2008). A similar study found that the duration of exclusive breastfeeding was associated with grey matter cortical thickness in both the inferior and superior parietal lobule in adolescents, which is a region comprised of mostly the association cortex (Kafouri et al., 2013). 
I found it more likely that the white matter effects reported in Issacs et al. (2010) and Deoni et al. (2013) were more pertinent to the proposed pathway for my dissertation proposal. Although also discussed later, the study by Issacs et al. (2010) found an association not only between increased breastfeeding and white matter volume, but also an association between white matter volume and IQ. This is in contrast to Kafouri et al. (2012) which did not find a correlation between cortical thickness and intelligence. Since my proposed model ultimately predicted that SDB would be correlated with neurocognitive measures (such as IQ), it was more likely that any proposed mechanism would involve white matter development. Furthermore, Deoni et al. (2013) found a relationship between breastfeeding duration and the microstructure of the white matter, suggesting perhaps not only a volumetric effect but also one of axonal quality. Finally, although Issacs et al. (2008) had found a correlation between nutrition and caudate volume in the brain, this effect was only associated with early diet not necessarily the full experience of breastfeeding, and as such inferences taken from that study for the purposes of this dissertation must be limited.

\subsubsection{Breastfeeding and Neurocognitive Outcomes}

Studies much more commonly, at least in breastfeeding research, have relied on maternal questionnaires for infant feeding methods information and inferring brain function from neurocognitive measures. Breastfeeding has been repeatedly correlated with later increased performance on neurocognitive measures in humans (J. W. Anderson et al., 1999; Horwood \& Fergusson, 1998; Isaacs et al., 2010; Kramer et al., 2008; McCrory \& Murray, 2013; Mortensen et al., 2002), particularly on IQ tests (Horwood \& Fergusson, 1998; Isaacs et al., 
2010; Mortensen et al., 2002). Additionally, increased duration of breastfeeding is likewise correlated with increased academic performance (Horwood \& Fergusson, 1998; Oddy, Li, Whitehouse, Zubrick, \& Malacova, 2011). Although these studies are by definition correlational, many of them have controlled for covariates such as age and socioeconomic status. The sheer volume of research in this area is suggestive of a possible causal link. Therefore, carefully controlled quantitative studies which investigate the brain in relation to these cognitive changes are necessary to help explain any possible mechanisms by which infant feeding methods may confer observable effects.

\subsection{Significance of White Matter in the Brain}

The human brain is a complex and magnificent organ which contains several layers of organizational structure. On a macrostrucural level, the human brain is comprised of the more evolutionarily conserved brain stem, subcortical structures, and the "newly" evolved neocortex. The brain stem, specifically the medulla, pons, and midbrain are responsible for life-critical functions such as respiration, cardiac function, sleep, and certain neural reflexes such as pupil dilation and restriction. Moving in the superior plane brings us to the subcortical structures such as the thalamus, hypothalamus, hippocampus, amygdala, caudate, globus pallidus and putamen. Generally, these structures have a significant role in many, many cognitive functions, but to name a few include hunger, thirst, reproduction, short term memory consolidation, anger and aggression, and strong emotional experience. Most importantly in the context of my dissertation, the sub-cortex is filled primarily with the axonal connections, or "wiring", between neocortical neurons and certain subcortical regions. The axonal connections are referred to as 
the "white matter" in the brain, whereas the cell bodies (Soma, dendrites, etc.) in the cerebral cortex are referred to as the "grey matter". These cortical regions in the brain are responsible for probably what most of the lay population would consider to be the functions that separate humanity from other animals. These functions include logic, decision-making, inhibition and self-control, planning and analytical thought (prefrontal and frontal regions), sensory integration and motor function, speech processing and verbal communication (posterior frontal and temporal regions), spatial reasoning and function, event association (parietal regions) and visual processing (occipital region), among others (Johnson, 2001).

The behaviors associated with grey matter are well documented, but perhaps unknown to most of the lay population, white matter also plays a large role in cognition and behavior. Multiple sclerosis, an autoimmune disease which attacks the myelin sheath in the brain, can produce unpredictable and unusual behaviors and phenotypes depending on which region of white matter is under attack (Trapp \& Nave, 2008). In this subsection the white matter anatomy, neuroimaging methodology, associated behaviors and neurocognitive functioning, possible developmental disorders, and plasticity will be discussed.

\subsubsection{Anatomy and Function}

As white matter really just represents the axons of other neurons in different areas of the brain, along with the associated glia that support and myelinate them, these regions can be viewed functionally as regions responsible for signal transduction between neural systems in the brain. The "white" that is observed in this tissue, at least from the post-mortem brain, actually comes from the lipid layers that insulate and myelinate them which are produced from 
the central nervous system oligodendrocytes. Generally, axons that are heavily myelinated (having more myelin- a fatty substance which insulates the axon and assists in the continued propagation of action potentials) are able to transmit signals more efficiently than axons that are more lightly myelinated and, as such, a greater volume or density of white matter is assumed to reflect greater signal transmission and communication between neural system axons that are linked in that region, since the white matter volume and density is primarily a function of myelin.

\subsubsection{Imaging and Methodology}

The methodology used to investigate white matter volume and anatomy in humans relies heavily and primarily on $\mathrm{MRI}$ as opposed to other imaging modalities. This is partially because white matter is located deeper in the brain and therefore surface only modalities are not effective (EEG, NIRS), and partially due to the spatial and temporal resolution necessary to image white matter, as well as the intrinsic mechanism in how MRI works compared to other imaging modalities.

The majority of white matter imaging relies either on volumetric analyses, which infer white matter function from the sheer volume of tissue labeled as white in any given region, or DTI, which is a measure of water diffusion through white matter tissues. In some sense the volumetric vs. diffusion tensor imaging can be viewed as a quantity vs. quality approach, as DTI attempts to measure the underlying myelination and white matter microstructure quality from the diffusion of water in these regions. It is important to note that although DTI is assumed to represent white matter integrity and quality, this is not a direct measure of it. Although white 
matter volume is commonly used today to assess brain structure, DTI is rapidly growing as an MRI brain imaging technique.

\subsubsection{Association with Neurocognitive and Behavioral Outcomes}

Few people would find white matter fascinating unless white matter actually can affect cognition, behavior, or some recognizable disease. Increased white matter volume is correlated with increased IQ scores in children (Isaacs et al., 2010) and adolescents born preterm (Northam, Liégeois, Chong, Wyatt, \& Baldeweg, 2011). Additionally, fractional anisotropy (FA) scores calculated from DTI (measure of directionality of water diffusion) indicate that the development and maturity of certain white matter regions (frontal and temporal regions) may be correlated with neurocognitive performance in children and adolescents (Nagy, Westerberg, \& Klingberg, 2004). Other studies have reported similar findings between white matter development and maturity and cognitive performance (Deutsch et al., 2005; Liston et al., 2006; Mabbott, Noseworthy, Bouffet, Laughlin, \& Rockel, 2006). Infants born pre-term who had abnormalities in white-matter structure had more severe neurocognitive impairment at age 4-6 compared to pre-term infants without these abnormalities (Woodward, Clark, Bora, \& Inder, 2012). However, and perhaps most importantly in this study, the pre-term infants without abnormal white matter structure had no difference in later neurocognitive performance compared to healthy controls, which is suggestive that white matter maturation might be directly involved in observed difference between neurotypical and preterm children. Together, these studies suggest that enhanced myelination of existing axonal connections and perhaps 
the addition or expansion of such connectivity between brain regions may have an important role in neurocognitive and behavioral development in pediatric populations as they age.

\subsubsection{Association with Developmental Disorders}

Examination of white matter abnormalities and microstructure in the context of certain developmental disorders at this point is somewhat speculative and controversial. Nevertheless, there are studies which have linked white matter characteristics to disorders such as autism spectrum disorders (ASD) and attention deficit hyperactivity disorder (ADD and ADHD). There is data to support that the developmental trajectory of white matter in ASD infants is different than healthy controls (Wolff et al., 2012). Specifically, when looking at FA values in 6 month old infants (before meeting ASD criteria) it was found that those who would eventually meet ASD criteria at 2 years of age actually had higher FA scores, but that these FA scores changed little over time and ultimately resulted in lower FA scores at 2 years old compared to controls. This study was able to find that white matter differences could actually precede the development of ASD criteria, suggestive of a possible mechanistic link between ASD and white matter. Other studies investigating ASD and white matter have found that certain white matter regions have lower FA values, and therefore assumed reduced development and maturation, in children, adolescents, and adults with autism (Keller, Kana, \& Just, 2007; Shukla, Keehn, \& Muller, 2011). This collection of work may indicate that early observed differences in white matter could persist into later childhood and adulthood.

White matter maturation may also be linked to ADHD. Among children and adolescents, it has been found that individuals with ADHD have white matter abnormalities as measured by 
DTI FA scores (Ashtari et al., 2005; Hamilton et al., 2008; Silk, Vance, Rinehart, Bradshaw, \& Cunnington, 2009) as well as in adults who had childhood ADHD (Makris et al., 2008). Generally, these studies indicate that white matter FA scores are decreased in ADHD, although not always (Silk et al., 2009). Although there is a general consensus that at least some of the white matter regions have decreased integrity in children with $A D H D$, what regions exactly are implicated can differ. Two regions which have been replicated in multiple studies include the cingulum (Makris et al., 2008; Silk et al., 2009) and superior longitudinal fasciculus (Hamilton et al., 2008; Makris et al., 2008; Silk et al., 2009), both of which are major white matter tracts in the brain. Although more research needs to be done in this area to draw definitive conclusions, these studies lay the groundwork for more neuroimaging to be conducted on certain developmental disorders. Although children with SDB often have hyperactive and ADHD-like symptoms (Chervin et al., 2002; Chervin, Ruzicka, Archbold, \& Dillon, 2005; Jan et al., 2011; Owens, 2009), investigation between possible common neurological outcomes remain controversial.

\subsection{Rapid Eye Movement (REM) Sleep and Brain Development}

Thus far I have concentrated primarily on the effects of SDB on children, the effects of infant feeding methods on children, and the significance of white matter. However, what I have not yet articulated is actually the central question of my dissertation: how can sleep actually have an effect on the developing brain? Why, mechanistically, would breastfeeding improve SDB, and why would this have any effect on sleep, specifically REM sleep? These are a few questions I aim to answer in this section. 
REM sleep is a stage of sleep quite distinct from the other NREM stages. Characterized by rapid, darting movements of the eyes, low muscle tone, and functional paralysis, it has fascinated researchers for decades, being the first stage of sleep scientifically studied (Aserinsky \& Kleitman, 1953a, 1953b, 1955a, 1955b). One of the early observations made about REM sleep is that the amount of sleep in newborn infants consists of a huge amount of the total amount of sleep ( $50 \%$ ) and diminishes very quickly, falling to about $25 \%$ by even $2-3$ years of age (Roffwarg et al., 1966). This observation, coupled with behavioral and eeg observation during REM sleep in infants has led to the hypothesis, called the ontogenetic hypothesis, that REM may actually play a critical developmental role in the early nervous system (Marks et al., 1995; Roffwarg et al., 1966). This hypothesis is further supported by the significant volume of literature pertaining to REM-dependent learning and memory, although these were demonstrated in adult populations (Karni, Tanne, Rubenstein, Askenasy, \& Sagi, 1994; Smith, Nixon, \& Nader, 2004). Both human studies of sleep deprivation and invasive animal research has also furthered this hypothesis (Roffwarg et al., 1966). Therefore, in addition to the observation of a high proportion of REM sleep early in development, REM sleep has also been associated with learning and memory, both of which require synaptic plasticity and changing neural networks in the brain.

The ontogenetic hypothesis states that REM sleep may promote neural maturation in the developing nervous system through intense electrical discharges from neurons within the brain (Roffwarg et al., 1966). Indeed, activity during this stage can be vigorous and is thought to originate in the pons, some coming up through the thalamus and some projecting straight into the cortex (Roffwarg et al., 1966). Since it has been shown that a lack of neural stimulation can 
prevent structural development and neural network maturation, and that proper stimulation and neural activity can produce the opposite -- well developed and responsive networks (Katz \& Shatz, 1996) -- it may be that REM-specific activity during sleep provides a mechanism for the development and maturation of critical networks. Furthermore, the activity and stimulation provided during REM sleep may spare developmentally critical circuits from synaptic pruning that necessarily occurs in early childhood, as stimulated circuits will not be eliminated as opposed to rarely used circuits and connections during the normal developmental process (Katz \& Shatz, 1996; Roffwarg et al., 1966).

Maturation of neural networks induced through REM could be achieved through the production of additional connections, or through the myelination of existing connections (Katz \& Shatz, 1996; Roffwarg et al., 1966). Since mature healthy circuits are almost always myelinated, and thickening of the myelin is localized around the axon, myelin is actually a great marker to measure neural network growth and maturation. Since the "white matter" in structural MRI scans originate from the lipid-rich signal from the myelin, MRI actually provides a great macroscopic measurement white matter maturation in the brain. This will become very important for my dissertation.

Where SDB fits into the question of REM sleep's importance in brain development and white matter is related to its ability to disrupt sleep. It is my contention that SDB sufficiently fragments sleep to such an extent that sleep architecture will be disrupted, preventing the normal healthy progression through sleep stages and reducing the overall amount of REM sleep, as it is typically present at the very end of the sleep cycle. Therefore when SDB is present individuals should also have a reduced quantity REM sleep which is fragmented. I hypothesize 
that this effect will manifest in children as reduced global white matter connectivity, as disrupted REM sleep will insufficiently stimulate important neural networks.

Finally, what about breastfeeding? How, mechanistically, would breastfeeding reduce the severity of SDB? Unfortunately, the precise mechanism for how this could occur, if it occurs, is not known. However, two hypotheses exist for how it might be possible (MontgomeryDowns, 2013; Montgomery-Downs et al., 2007). The first hypothesis proposes a morphological change in an infant's airway due to prolonged breastfeeding as opposed to bottle-feeding. According to this theory, the morphological changes associated with breastfeeding presumably restructure and move soft tissues around the upper airway in such a fashion that it reduces the likelihood of collapse during sleep (Montgomery-Downs, 2013; Montgomery-Downs et al., 2007). This could be possible due to the unique muscle coordination that must be achieved during suckling from breastfeeding as opposed to bottle-feeding, which could promote healthy jaw formation and other beneficial structural changes around the airway (Montgomery-Downs, 2013; Montgomery-Downs et al., 2007).

A second hypothesis centers around the introduction of breastmilk itself, rather than the structural changes associated with suckling (Montgomery-Downs, 2013; MontgomeryDowns et al., 2007). In this scenario, it could be possible that breastmilk helps to provide immune support to the infant that reduce infection in the airway. Overall reduction of infection, and severity of infection, would thus also reduce inflammation and possible hypertrophy or airway tissues that are often associated with SDB (Montgomery-Downs, 2013; Montgomery-Downs et al., 2007). Reduction of inflammation and airway tissue growth due to chronic infection may provide a mechanism for how breastfeeding could reduce SDB. 
Ultimately, independent of mechanism, I predict that pediatric individuals with SDB who were breastfed will not only have reduced disease severity, but increased global white matter connectivity through preservation of internal REM sleep stimulation.

In summary, I argue in this dissertation that breastfeeding will be positively correlated with reduced SDB, or healthier sleep, and that reduced SDB will likewise be correlated with better quality and quantity of REM sleep. More REM sleep should translate into better maturation of neural networks and white matter development, which should be able to be detected via MRI. I hypothesize that SDB and white matter development are linked, but this linkage is indirect and relies on the theoretical importance of REM sleep on development.

1.7 Putting it All Together: Breastfeeding, Sleep-Disordered Breathing, and White Matter

\section{Development}

The purpose of this doctoral dissertation was to attempt to reconcile and integrate infant feeding methods, sleep-disordered breathing, white matter development, and neurocognitive performance into a single overarching developmental hypothesis which attempted to infer some mechanistic pathway for the purposes of intervention for children with unhealthy sleep. In sum, breastfeeding may have positive benefit in reducing SDB severity, thus contributing to more healthy sleep (Montgomery-Downs et al., 2007). The amount of breastmilk consumed or the duration of breastfeeding until weaning seems to be related to white matter changes in the brain (Deoni et al., 2013; Isaacs et al., 2010). Breastfed children, compared to children who have never been breastfed, often show significant improvements in neurocognitive function, and quite often IQ scores (J. W. Anderson et al., 1999; Horwood \& 
Fergusson, 1998; Isaacs et al., 2010; Kramer et al., 2008; McCrory \& Murray, 2013; Mortensen et al., 2002). It is also true that children with SDB have worse performance on neurocognitive tasks (Bourke et al., 2011a; Chervin et al., 2002; Halbower et al., 2006; Jan et al., 2011; Karpinski et al., 2008; Kohler et al., 2009; Perfect et al., 2013) than healthy controls. Effects that have been reported in particular have been deficits in executive function (Bourke et al., 2011a; Halbower et al., 2006; Karpinski et al., 2008; Kohler et al., 2009), academic performance (Chervin et al., 2002), and various problems with behavior (Chervin et al., 2002; Jan et al., 2011; Perfect et al., 2013). Given that the deficits seen from a lack of breastfeeding are very similar to deficits seen from SDB, further investigation into a possible shared mechanism involving neurodevelopment may be reasonable, and will be examined in this dissertation.

The major consequences of pediatric SDB, or SDB at any age, are the chronic and persistent sleep fragmentation caused by hypoxia, and the oxygen desaturation associated with said hypoxias. One of the suspected results of chronic sleep fragmentation is a general disruption of sleep architecture (Redline et al., 2004; Short \& Banks, 2014). Since the lighter stages (N1 and N2) of sleep have an earlier onset than N3 sleep and REM sleep, which usually require the sleeper to have been asleep for at least a few minutes, if not longer, it is logical that a disorder which invokes frequent arousals and sleep disruption would severely hinder progression of sleep into these later stages. The idea that SDB may reduce REM sleep quantity has been supported by literature (Redline et al., 2004). Therefore, beneficial effects from REM sleep that may ordinarily be conferred in healthy children might be denied to children with SDB in some capacity if the disease is present early in development. 
In order to fully conceptualize this project, I would like to refer the reader to Fig 1. In its simplest form, I proposed that an increased duration of breastfeeding (the length of time until an infant is completely weaned from breastmilk) would be positively correlated with white matter development as has been previously reported (Deoni et al., 2013; Isaacs et al., 2008). I proposed that the main constituents of this relationship may involve factors such as introduction of enhanced nutrition, immune support, mother-infant bonding, and other miscellaneous factors. Nevertheless, I further proposed that at least of portion of this relationship can be attributed to sleep. Increased breastfeeding duration has been negatively correlated to SDB severity (Montgomery-Downs et al., 2007), and as such I suspected that a lack of breastfeeding (or decreased duration of breastfeeding) would be correlated with increased SDB severity, or more disordered sleep. Since I predicted that healthy sleep is positively correlated with increased white matter development, a general lack of healthy sleep induced by SDB should decrease white matter development through chronic fragmentation of sleep and deoxygenation of brain tissue. In short, I predicted that the main effect of breastfeeding on white matter development would involve various other factors, but there would at least be a partial mediation effect of sleep on white matter, which can be influenced by breastfeeding itself.

The full model for my dissertation can be explained in Fig 2. In addition to what was explained previously, this model goes into further detail in that it proposes by which mechanism SDB severity causes white matter changes. As articulated by Roffwarg et al. (1966), REM sleep may have a developmentally important role in brain development through the repeated stimulation of cortical neural networks which are critical for development. Such 
stimulation of these networks is thought to spare the connections from later developmental pruning. Therefore, REM sleep may provide a developmental function in which it strengths and assists in maturation of important neural connections, leading later to their myelination. If true, such myelination and maturation of white matter neural processes should be able to be macroscopically measured using MRI. Referring back to Fig 2, I predicted that a higher severity of SDB would be correlated with a decrease in REM sleep (and continuity) due to chronic obstruction and a lack of proper progression through sleep staging. Subsequently, I believed that the decrease in REM sleep specifically would be associated with decreased white matter development. Furthermore, it was predicted that the decreases in white matter development would be correlated with decreases in neurocognitive measures. In essence, this project attempted to explain how sleep may or may not affect real brain changes, and how these changes might ultimately manifest into behavior.

\subsubsection{Specific Aim 1}

The purpose of the first Specific Aim in this dissertation was to examine the relationship between breastfeeding, SDB, and white matter changes in the brain. In order to investigate this, approximately 25 children (4-8 years old) with SDB were recruited from a local sleep clinic to undergo several structural MRI scans. White matter was assessed by using corpus callosum (CC) volume, as the $\mathrm{CC}$ is the dominant interhemispheric structure in the brain. I predicted that SDB mediates the relationship between breastfeeding and white matter development (see Fig 1). The central hypothesis for this Specific Aim was that breastfeeding duration and CC volume is positively correlated, but that at least a portion of the variance is attributed to SDB. CC volume 
was measured by summing together the central $13 \mathrm{~mm}$ of individuals participants. Additionally, neurocognitive measures were also collected for these participants, and as such correlations between breastfeeding, CC volume, and cognitive performance were examined.

\subsubsection{Specific Aim 2}

The goal of Specific Aim 2, like Specific Aim 1, was to investigate the relationship between infant feeding methods and white matter changes in the brain. However, unlike in the previous aim, only healthy children (do not have SDB) were recruited for MR imaging. Twentyfive participants were recruited and volumetric measurements on the $\mathrm{CC}$ in the images were conducted. The central hypothesis of Specific Aim 2 was that breastfeeding is positively correlated with CC volume, as was proposed in Specific Aim 1. The key to Specific Aim 2 is that, since none of the children had a diagnosis or symptoms of SDB, the correlation between breastfeeding and CC was hypothesized to have a lower magnitude than it was in Specific Aim 1 due to the loss of the partial mediator SDB (refer to Fig 1). Ergo, although a similar effect was predicted in both Specific Aims 1 and 2, the overall effect was predicted to be stronger in Specific Aim 1 since the children in that sample suffer from SDB. CC volume was measured the same way as it was in Specific Aim 1. Other methodological procedures will be discussed in detail in the following sections.

\subsubsection{Specific Aim 3}

The purpose of Specific Aim 3 was to investigate the possible link between infant feeding methods, SDB severity, and amount and architecture of REM sleep in a very young 
population. In order to obtain as much information of possible, this aim was divided into two different experiments. The first experiment consisted of direct polysomnography of infants and children (6 months- 6 years) recruited to the lab and assessed for both SDB and general sleep architecture. The second experiment utilized a longitudinal survey for which PSG measures were previously acquired for 8-9 month old infants in order to obtain infant feeding methods information, as well as follow-up on SDB related questions 11-12 years later to look at longer term effects.

\subsection{Methods}

\subsubsection{General Measures}

\subsubsection{Magnetic Resonance Imaging (MRI)}

Magnetic resonance imaging (MRI) is one of the newest and widely used neuroimaging techniques. The core principle of MRI relies on the discovery of nuclear magnetic resonance (NMR) years earlier which, from a basic perspective, describes that atomic nuclei are able to interact with and absorb radiofrequency, and that this radiofrequency can alter the resonance of atomic nuclei at a precise frequency that is influenced by several factors (Blink, 2010; Haacke, Brown, Thompson, \& Venkatesan, 1999; Schild, 1999). Although a full review of MRI physics is outside the scope of this dissertation, a brief review is warranted.

In MRI, the resonance frequency of an atomic nucleus is determined by the Larmor equation, which takes into account both the strength of the magnetic field and the gyromagnetic ratio (a ratio that accounts for the differences in resonance frequency between different nuclei and is determined by nuclear composition). It is important to note that atomic 
nuclei have their own unique resonance frequency. In MRI (unlike NMR) the major nuclei that are measured are the hydrogen atoms in water. Although the resonance frequency to disrupt the spin of a hydrogen atom in a water molecule will be the same for every water molecule (assuming the magnetic field strength is constant), the frequency required to disrupt a hydrogen nuclei in, for example, glucose, would be different. Therefore, specific resonance frequencies enable targeted imaging of the brain based on the concentration of water molecule distribution throughout the tissue.

Spin is an inherent property common to all protons (hydrogen nuclei) in which the charged particle rotates along an axis, generating its own local magnetic field (Blink, 2010; Haacke et al., 1999; Schild, 1999). Since these protons have a magnetic field themselves, they are sensitive to external magnetic fields as well. Therefore, when these protons enter the magnetic field that is generated in MR imaging, their own magnetic field will be aligned along that of the bore of the MRI. Although these nuclei are now aligned with the MRI magnetic field, they are still resonating at their own resonance frequency. The resonance of these nuclei can be thought of as a "wobbling" of the local magnetic alignment around the axis of the MRI magnetic alignment.

The central magnet in the MRI produces the constant magnetic field, also known as B0. The MRI can also contain various gradient coils, which can subtly alter the B0 in different locations along the longitudinal axis of the magnet. This allows for signal localization (changing $\mathrm{BO}$ changes the resonance frequency, and therefore attenuates the incoming signal- will be talked about more a little later). Another important component of the MRI includes the radiofrequency (or RF) coils. These coils are able to transmit electromagnetic energy in the 
radiofrequency range out into the bore. This concept is critical to the function of MR imaging: the radiofrequency used by the RF coils must match the resonance radiofrequency of the nuclei of interest (in this example, hydrogen nuclei in water molecules), as only the targeted nuclei are the ones which will produce a response (Blink, 2010; Haacke et al., 1999; Schild, 1999).

The fact that protons will only become excited (absorb radiofrequency, disrupt alignment with the main magnetic field) at a precise frequency (dictated by Lamor frequency) allows for specific targeting of areas in the body, although the reason for this may not be obvious for non-MRI experts. The gradient coils which are contained around the bore of the magnet subtly change the BO across the longitudinal axis of the MRI. However, because BO is part of the Lamor equation, it means that each proton at specific locations along the longitudinal axis of the MRI has its own specific resonance frequency. Therefore, by subtly changing the radiofrequency emitted by the RF coils one can target specific hydrogen nuclei in water in different areas of the body. The range of frequency applied (through electromagnetic near-field effects; not radiating radio waves) can also be adjusted to determine how large of an area to excite (Schild, 1999).

As it turns out in MR imaging, producing a coherent image isn't just about receiving RF energy after an RF exciting pulse is released; the receipt of this energy is time-sensitive (Blink, 2010; Haacke et al., 1999; Schild, 1999). Two commonly used imaging methodologies in MRI include T1-weighted imaging and T2-weighted imaging. After particular protons are excited by an RF pulse, they not only become misaligned with the main magnetic field, but also briefly become aligned in phase (basically meaning all the local magnetic fields of the excited protons all point in the same direction) perpendicular to the longitudinal magnetic field. After a short 
amount of time, these protons will "relax" or lose phase coherence (the local magnetic fields of the excited protons begin to no longer point in the same direction) changing the local magnetic field and transmitting RF frequency to the receiving coils. The amount of time it takes for these protons to become sufficiently dephased from one another in the plane perpendicular to the main magnetic field results in the $\mathrm{T} 2 *$ (T2 star) relaxation time. Sometimes, a refocusing pulse or "echo" pulse will be used to allow the protons which lose phase coherence to regain it again after a brief period of time. The time it takes for these out of phase protons to regain phase along the echo pulse axis is the T2 relaxation time. In T2-weighted imaging, either a single spinecho or multi-echo approach could be used, resulting in scans that can last significantly longer than $\mathrm{T}_{2}$ * relaxation with a single pulse. Receiving coils are able to collect the RF signal that is transmitted due to nuclear relaxation, and hence 3 dimensional reconstruction techniques can later be applied based on the relaxation of interest ( $\mathrm{T} 2 *, \mathrm{~T} 2$, or $\mathrm{T} 1)$. The receipt of this RF frequency by the RF coils (through induced electromotive force (EMF); not radiating radio waves) is ultimately what will produce different intensities in imaging space, producing a coherent image after reconstruction (Schild, 1999). Higher signals in particular regions will correspond with different brain tissues, based on water density, allowing for precise tissue differentiation.

In T1-weighted imaging, the proton relaxation is not measured as a loss of phase coherence as is the case in T2 imaging, but rather the total time that it takes the protons to lose enough excitation energy to flip completely back and realign with the magnetic field (Blink, 2010; Haacke et al., 1999; Schild, 1999). That is, the time required for T1 relaxation is quite longer than that of $\mathrm{T}^{*}$, as the protons must first lose phase coherence and have random 
distribution in the perpendicular axis $(\mathrm{T} 2 *)$ before relaxing further and realigning themselves with the main axis of the magnet. Counter intuitively, T1-weighted scans can often actually be shorter than the T2-weighted scans, since in T2 imaging the protons can be given multiple pulse echos to optimize tissue differentiation.

As it turns out, the relaxation time of the hydrogen nuclei in water will differ based on its surrounding environment. Water in the cerebrospinal fluid (CSF) and other areas of the brain where water is largely unbounded will be able to move around more freely, leading to longer T1 and T2 relaxation times (Blink, 2010; Haacke et al., 1999; Schild, 1999). In contrast, water molecules that are contained within lattice and matrices, such as water within myelin tissue, will have more interaction with the surrounding matrix and result in shorter T1 and T2 relaxation times. As a result, designing imaging sequences that take advantage of these different relaxation times can produce an image with excellent soft tissue contrast between the CSF, grey matter, and white matter within the brain. As a rule of thumb, T1-weighted imaging is better for looking at grey matter/white matter tissue contrast and white matter volume measurements, while T2-weighted is more sensitive to abnormalities within the tissues themselves while slightly sacrificing grey/white matter contrast. Since my particular research question in this dissertation involved white matter measurements, I used T1-weighted scans when possible.

In summary, structural MRI is a complicated endeavor but ultimately produces high resolution images with excellent soft tissue differentiation useful both clinically and in research. In research, such images are extremely useful for conducting volumetric measurements and 
also examining tissue abnormalities in the brain. Although the most basic variant of MR imaging, this technique remains incredibly useful.

\subsubsection{Diffusion Tensor Imaging (DTI)}

DTI relies on diffusion-weighted images (DWI) that map the movement of water molecules throughout brain tissues. In particular, DTI analyses the diffusion of water in the brain per voxel both in totality and along particular vectors of interest within the bore of the magnet (Soares et al., 2013). The amount of water diffusion along a vector, or multiple vectors, can alter the magnetic field, resulting in an attenuated signal (signal change) that can be detected.

DTI usually involves the acquisition of several different parameters, but the most commonly reported in scientific publications involve mean diffusivity (MD) and fractional anisotropy (FA). Mean diffusivity reports the total amount of water diffusion present in a particular voxel, while fractional anisotropy is a measure of the directionality of this diffusion. FA values are reported between 0 and 1; 0 reflects diffusion which is equal in all vectors, while 1 reflects diffusion that is entirely encompassed within a single vector to the exclusion of all others.

Application and interpretation of DTI generally focuses on white matter microstructure and strength of connectivity within the brain (Soares et al., 2013). This type of imaging operates under the assumption that water molecules located within neural axons (the white matter) will be restrained by cellular membranes and myelin around the axons and result in diffusion along a particular vector. In CSF and grey matter, the assumption is that because water can more 
freely move around those water molecules will diffuse in more random directions and not along any particular vector. Therefore, white matter should be much easier to identify compared to other tissue types. Furthermore, when comparing between white matter tissues, it is thought that higher FA scores represent higher quality axonal connections with greater amounts of myelination, and perhaps even axons which are larger in diameter, all of which are thought to contribute to more complete connectivity and enhanced strength of connections in those areas of interest. Another underlying assumption is that axons which are more heavily myelinated or larger in diameter are so due to frequent usage, and that the enhanced structural aspect of these axonal characteristics are correlated with underlying functional components. If these assumptions are not met, the utility of DTI in such an applications would be severely limited.

\subsubsection{Polysomnography (PSG)}

The gold standard in sleep research, polysomnography (PSG) primarily draws from the same technology as conventional electroencephalography (EEG) through the use of conductive electrodes on the scalp which measure electrical potentials which originate from the brain. However, unlike traditional EEG, PSG overall requires fewer sensors on the head and more sensors on the face and body. Using several different signal sources, differences in sleep state can be ascertained (Berry, Brooks, et al., 2015).

Before the actual application and use of PSG setup the electrode placement must be considered. There are several different methods that can be employed for placement, but in PSG the international 10/20 measuring system is the most commonly used standardized system (Leary, 2007). Briefly, this system relies on measurements of the head to determine the precise 
placement of these electrodes, which are calculated based on distance between head landmarks (from the indentation at the top of the nose between the eyes [nasion] to the back of the head at the bulge [inion]; between the indentations above the ear canal [preauricular points]; head circumference). A typical PSG-EEG montage will include at least bilateral electrodes occipitally and centrally, but can also include electrodes placed frontally. In the current study (Aim 3), the international 10/20 system coupled with 6 exploratory electrodes (bilateral occipital, central, and frontal) were used, which is the standard plus two optional exploring electrodes (frontal).

In addition to head EEG placement, bilateral electrooculography was used to measure eye movement and score REM sleep. Chin electromyography is used to measure general muscle tone and is also important in the scoring of REM and between sleep and wake. Bilateral anterior tibialis electromyography was implemented to score leg movements and help indicate arousals. Electrocardiogram was employed mainly for safety reasons.

Respiratory effort was measured using respiratory inductance plethysmography, a technique which relies on piezo-crystals which, when stressed or stretched, will generate voltages (Leary, 2007). These crystals are applied to sinusoidal bands which are wrapped around the body, and are stretched and relaxed as the body alters circumference in response to breathing, generating an indirect measure of effort. Respiratory belts were placed just beneath the ribcage and around the thorax.

Airflow was measured via nasal cannula with pressures sensors, CO2 sensors (common addition for pediatric studies; Berry et al., 2015), and thermistor (measures breathing through temperature changes). Respiratory scoring was based on waveform (Berry, Brooks, et al., 2015). 
Snore microphone was attached to check for PSG-validated snoring, and pulse oximetry was applied to measure blood oxygen level, which takes advantage of the different infrared absorption properties of hemoglobin when bound and unbound to oxygen.

All data (in aim 3) were collected with detachable multichannel bedside amplifiers (Embla N7000; Natus Medical Incorporated, Pleasanton, CA, USA) and associated patient unit.

\subsection{Specific Aim 1}

\subsubsection{Description of Procedures}

The sample used for the first Specific Aim were 29 children between 4 and 8 years old and for whom there was suspicion of SDB at a local sleep clinic. Children who were scheduled to receive clinical screening and possible diagnosis of SDB at Dr. Clawges' pediatric sleep practice at the WVU Sleep Disorders Center were recruited and evaluated for eligibility to participate. Recruited participants had varying durations of breastfeeding and methods of feeding, as well as severities of SDB (as measured by AHI). The research personnel were able to acquire a full overnight polysomnography report of the participants that was carried out and generated by Dr. Clawges's pediatric sleep practice (WVU Sleep Evaluation Center).

Parents of qualifying children were approached in the waiting room of their clinical visit and, if they agreed to participate, were scheduled to come to the MRI scanner at the Center for Advanced Imaging in Ruby Memorial Hospital associated with West Virginia University at a later date. Upon the day of arrival, participants and their parents were taken to a research laboratory and had the protocol explained and any questions answered. Adult consent and child assent were then administered. It was immediately after consent that infant feeding 
methods (Appendix A), breastfeeding duration, and other general demographics (Appendix A) were acquired regarding the child participant via parental report (often maternal). Behavioral information for the purposes of screening and participant treatment (Appendix B), and an MRI safety form (Appendix C) were also completed. It is important to note that investigators were blinded to breastfeeding status and SDB severity until after MRI analysis was complete. Blinding was achieved in these preliminary stages by having the parents of participants place their answers into sealed envelopes.

Following consent and general paperwork the participants were taken to a mock scanner to become acclimated to the MRI environment. Parents were also present in the room and assisted the researchers in comforting the participant. The mock scanner was approximately the same as the actual MRI scanner dimensions, with a slightly smaller bore diameter and motorized table to move people in and out. The participants were asked to lay down on the table, place their head in the mock head coil, and were moved down to the center of the bore using the table motor. Once in the center, a researcher would then move to a small window where the participant could see them and read them a children's story (often "The Poky Little Puppy" by Janette Sebring Lowrey), which could vary based on the child's preference. After the story, participants were then removed from the bore and then either decided they wished to continue to the actual MRI scanner, or stop the study and go home (parents would receive a 50 USD gift card as compensation in the latter case, while children were given a small age-appropriate toy (1-5 USD in value)). Exact procedures and chronology may have varied based on the level of cooperation of the pediatric participant. 
Subjects were then guided to the Center for Advanced imaging which houses the 3 Tesla Siemens Verio MRI scanner (Iselin, NJ). Parents were asked to stay back in the control room (where they could still see their children through the viewing window) while the participants were asked to lay down on the motorized table for the MRI after confirming the child had no metal in/on their person. Initial setup for the participants included head support, leg support, hearing protection, a heated blanket if desired, etc. A mirror reflecting the image of a television screen within the MRI room is also attached to the head coil to allow the participants to watch a movie of their choice during the imaging, the audio of which was streamed through dualpurpose hearing protection/audio streaming ear plugs. Participants were not given a squeeze ball for quick exit; instead a researcher remained in the scanning room at all times and monitored the participant during the scans. Although the participants were all asked to remain as motionless as possible, the researcher monitoring the participant during the scans would sometimes remind the child to stay still if they were moving too much by gently touching their leg. Children were not sedated at any point during the study.

Participants were all placed in the supine position and aligned along the Frankfort plane as much as possible. An 8-channel head coil was used for every participant, while a 4 channel neck coil was used only if the child's entire head and neck did not fit inside the head coil (typically used on older children). A localizing scan was used to ensure the participants head and neck were completely within the field of view.

There were two general classifications of T2-weighted protocols which were used; a slower, better resolution T2 scan ( 6 mins) for cooperative subjects and a faster, poorer resolution T2 scan ( $\sim 3$ mins) for less cooperative subjects at the discretion of the research staff. 
Although exact scanning parameters did vary throughout the course of the study, generally speaking the slower T2 scans were acquired in either the sagittal or axial plane with $1.04 \times 1.04$ $x 2 \mathrm{~mm}^{3}$ voxels, with slice thickness in plane of acquisition being the worst resolution. Other scanning parameters (repetition time $(T R)=9180 \mathrm{~ms}-10560 \mathrm{~ms}$; echo time $(T E)=70 \mathrm{~ms}$, Flip angle $=150$, matrix size $=192 \times 192, \mathrm{FOV}=199 \times 199 \mathrm{~mm}^{2}$ ) were kept as constant as possible, but did vary on occasion.

The other class of T2-weighted scan (faster scan) also had the same caveats of variability as the slower $\mathrm{T} 2$, but generally had constant parameters $\left(1.04 \times 1.04 \times 3 \mathrm{~mm}^{3}\right.$ voxels, repetition time $=750 \mathrm{~ms}$, echo time $=9.4 \mathrm{~ms}$, flip angle $=150$, matrix $=192 \times 192$, FOV $=199 \times 199 \mathrm{~mm}^{2}$ ). Again, the T2 image chosen was based on the cooperative nature of the participants. Many participants received multiple $\mathrm{T} 2$ scans, sometimes both fast and slow, and the best scan (by eye, at the discretion of a trained researcher [me]) was chosen for analysis. A table of the different T2 scans analyzed and corresponding participant is presented in Appendix D.

The T1 (magnetization-prepared rapid gradient-echo; MPRAGE) scans had variable parameters for different participants, partially due to trying to find the optimal scanning parameters for imaging children. Generally, the images were acquired in the axial plane (two of the participants were acquired in the sagittal plane; 571 and 885) with voxel sizes of $0.625 \mathrm{x}$ $0.625 \times 1.5 \mathrm{~mm}^{3}$. The two scans acquired in the sagittal plane were also this same voxel size, except with the resolution being worse in the sagittal plane also opposed to the axial plane in the axially acquired images. Three additional participants, although acquired in the axial plane, had voxel sizes of $0.45 \times 0.45 \times 0.9$ millimeter (993), $0.625 \times 0.625 \times 1.2$ millimeter (686) and $0.62 \times 0.625 \times 0.9$ (568). Despite the differences in acquisition plane and resolution, all scans 
but one shared the same repetition time $(T R=2300 \mathrm{~ms})$, echo time (TE= 3.77 $-3.81 \mathrm{~ms})$, flip angle (flip angle $=9)$, matrix size (matrix size $=384 \times 312)$ and field of view $(F O V=195 \times 240$ $\mathrm{mm}^{2}$ ). Only one participant (993) had different parameters (TR=1900 ms, TE= $2.99 \mathrm{~ms}$, Flip Angle $=9$, Matrix $=512 \times 448, \mathrm{FOV}=201 \times 230 \mathrm{~mm}^{2}$ ). MPRAGE scans were approximately 10 minutes in length. The MPRAGE selected parameters can be viewed in Appendix E.

The field of view for all of the participants was generally centered on the upper airway. Partially because of this, along with the consideration that researchers needed to make the scans as quick as possible to reduce burden on a pediatric population, the top portion of the brain was sometimes not included in the image. The reason for centering the image on the upper airway is that these images were also intended for a different project currently undertaken by the lab's primary investigator (my advisor, Hawley Montgomery-Downs). Although this omission in the scans created a challenge for data processing and normalization, the CC was often left intact thus arguably not affecting the study sample on that particular measure.

Once all of the scan sequences were acquired the participants were taken out of the scanner. Parents received a 165 USD gift card for their time and travel while participants were given a small toy. A gift receipt for the gift card was signed by both parents and investigators. Depending on the parents availability, the participants were then escorted to another area for behavioral and neurocognitive testing with a graduate research assistant (Pamela Tessier; also blinded to breastfeeding duration and PSG outcomes) either subsequent to the scanning or on another day. Total testing time was between 35-50 minutes and included a battery of seven assessments that are well-validated for this age group. These assessments 
mostly focused on executive functioning, as this type of functioning in particular has been welldocumented in its impairment in SDB pediatric populations (Archbold, Giordani, Ruzicka, \& Chervin, 2004; Karpinski et al., 2008). The neurocognitive tests employed during this session included the Color-Form Test/Progressive Figures (mental flexibility; Reitan \& Wolfson, 1985), the Day-Night Stroop Task (inhibition control; Gerstadt, Hong, \& Diamond, 1994), WoodcockJohnson Tests of Cognitive Abilities (WJ-III Exec) Executive Processes Cluster (strategic planning/mental flexibility; Taub \& McGrew, 2004; Woodcock, McGrew, \& Mather, 2001), the WJ-III COG Numbers Reversed (working memory; Taub \& McGrew, 2004; Woodcock et al., 2001), Peabody Picture Vocabulary Test 4th Edition (PPVT-4) (verbal IQ; Dunn \& Dunn, 2007), and the Behavior Rating Inventory of Executive Function (BRIEF; well-rounded measure of executive function; Gioia, Isquith, Guy, \& Kenworthy, 2000). Neurocognitive measures were scored by the same graduate research assistant (GRA) that conducted the tests (Pamela Tessier).

\subsubsection{Data Processing}

After image acquisition during the MRI session the raw digital imaging and communications in medicine (DICOM) files were transferred from the control station to the research laboratory. DICOM files for all scans were viewed for quality using the free DICOM viewing software OsiriX ${ }^{\mathrm{TM}}$ (version 3.7.1). After initial inspection, DICOM files for both the T2 and MPRAGE images were converted into "head" and "brik" files using the open-source software Analysis of Functional Neurolmages (AFNI; [Cox, 1996]). AFNI has a command called To3d, which can convert raw slice by slice DICOM files into a 3d image that can be interpreted 
using AFNI. All scans used for analysis were converted in this way. After conversion, these files were registered along the anterior commissure- posterior commissure (ACPC) line, but further registration into Talairach space did not occur when considering this pediatric sample. It did not seem appropriate to apply further registration using the Talairach transition to pediatric brains because the dimensions and accuracy of such transformation can be highly variable with a young population (Giedd \& Rapoport, 2010). It is important to note that the registration process resampled the native voxels from the T2 and MPRAGE scans into 1 millimeter $x 1$ millimeter $x 1$ millimeter voxels.

Once all of the scans were properly registered, manually drawn regions of interest (ROIs) were drawn around the CC of each individual on both T2 and MPRAGE scans by an investigator blinded to feeding methods and sleep outcome. All CC ROIs included the entire callosal body, but excluded areas such as the fornix and peripheral white matter. In order to measure as much of the $\mathrm{CC}$ as possible, the central 13 millimeters of the structure was measured in every individual. There is some precedence for using this measurement thickness (Frazier et al., 2012; Hardan et al., 2009), but mostly was chosen because it allows peripheral CC imaging but does not extend too far as to confuse general peripheral white matter with the CC.

I was blinded to the identity of the participants while drawing manual ROls, as well as other critical information such as infant feeding methods, sleep characteristics, general demographics, and neurocognitive measures. This was done using a unique 3-letter coding scheme that was totally independent from the experimental coding and was known only to the blinder (Caitlin Montgomery). This blinding was maintained while the second CC rater (Paula Webster) also performed the tracings. Both verbal and written instructions for ROI drawing 
were provided (Appendix F). The ROls were drawn by both raters (I had drawn all the participant's ROIs [four for intra-rater reliability]; Paula also drew four participants from aim1). Both inter-rater reliability and intra-rater reliability (only for me) were calculated using intraclass correlation coefficient (ICC) in Statistical Package for the Social Sciences (SPSS; [Appendix G]). The total intra-rater ICC for all 4 scans in aim 1 using a two-way mixed model with absolute agreement and average measures yielded an ICC $=0.997, p<0.001$. The interrater reliability measurements for all 4 scans yielded an ICC $=0.931, p=0.038$. Taken together, both the inter-rater reliability and the intra-rater reliability using intraclass correlation coefficient was found to be excellent (0.997 and 0.931, respectively). Although second measurements for the primary rater and measurements from the secondary rater was used to calculate ICC, ultimately only the primary rater's first measurement was used for analysis.

In addition to general blinding of the participant's identity, the raters were also blinded to any airway tissues that might create bias. Since the images in this aim included the upper airway of the children, and because I am a sleep researcher with intimate knowledge of how tonsillar and adenoidal size can influence sleep-disordered breathing, these tissues were masked using a simple numerical overlay which can be drawn in AFNI. The exact procedure for this can be viewed in Appendix $\mathrm{H}$.

The procedure for the actual drawing of the ROIs themselves was quite simple. The $3 d$ registered images were open and viewed in AFNI as the anatomical underlay. Immediately after this step, the overlay which contained the aforementioned blinding ROI for upper airway tissue was loaded onto the screen. The first slice to be considered for CC ROI measurement was the mid-sagittal slice, which is clearly labeled after registration. The CC was then subdivided into 3 
sections: anterior, central, and posterior in order to look more closely at particular areas of the CC. These subdivisions were manually determined by taking the anterior-most point of the CC on mid-sagittal slice and measuring the distance to the posterior-most point. The length of the CC was then sub-divided into 3 and beginning and endpoints for the 3 areas were determined. If the total length was not divisible by 3 , an extra voxel was given based on priority; anterior had first priority, posterior had second priority. For example, if the length of the CC was $68 \mathrm{~mm}$, then the anterior portion would be $23 \mathrm{~mm}$, the posterior portion $23 \mathrm{~mm}$, while the central portion would only be 22 millimeter in length on the central slice.

After the lengths of the three subdivisions were determined, a 2 dimensional CC ROI was drawn on the mid-sagittal slice. The overlay containing the blinding ROI had already been loaded, arbitrarily given a value of 4 to contrast with the 3 subdivisions of the CC. The anterior region was assigned a value of 1 ; the central region a value of 2 , and the posterior region a value of 3. This numerical system was maintained for all subsequent 2-dimensional ROls. With the sagittal plane as the main plane that was used to draw the ROls, the brain was moved through in one millimeter steps out to six millimeter from the midline in each direction, for a total of 13 millimeter thickness using 13 one-millimeter slices. Although the axial and coronal planes were used to some extent in drawing the ROIs, they were used much more heavily for post-hoc CC correction. After all 13 sagittal ROIs were drawn, the CC was viewed through the whole brain on both the other two planes. Typical areas that needed adjustment included the inferior-anterior regions, the inferior-central regions which could be confused with fornix and other tracts, and superior-posterior regions that were hard to differentiate from general white matter. After the CC ROIs were "finalized" the total volume for each subsection was determined 
using the "3droistats" command in AFNI which provides statistics for masked regions. This program will count the number of non-zero voxels (and separate them based on voxel value), and since all voxels were resampled to isotropic one millimeter voxels it was easy to determine the cubic millimeters of each sub-region. The total $\mathrm{CC}$ volume was calculated by adding all three of the sub-region volumes together. These data are presented in Appendices I and J.

In addition to manually drawn ROIs, I also used a semi-automated approach to determine CC thickness. This procedure involved using Freesurfer software (version 5.3; Fischl et al., 2004) to automatically determine the $C C$ boundary. Automated $C C$ measurements using the same software and procedure have been conducted (Shashi et al., 2012). However, since Freesurfer is only able to read T1 anatomical images, only the participants who had received an MPRAGE scan were included (therefore, most of the participants in this aim were excluded, since the $\mathrm{T} 2$ images were the most predominant). As a result, the sample size for this particular experiment in this aim was six (11 participants with MPRAGE, five failed automatic processing), with only four showing any strong indicators of SDB.

The first step in the semi-automated pipeline was to enter the raw files to be reconstructed into Freesurfer using the command recon-all. Recon-all is a particularly powerful tool which will convert the raw images into a 3-dimensional brain image, perform registration, skull-stripping, whole-brain segmentation (labeling of grey and white matter areas, including cerebellum and to some extent brain stem), image smoothing, and cortical parcellation and mapping. Although there are numerous setting and parameters that can be adjusted, in this application only the default settings were used. If any of the datasets failed in the reconstruction, they were excluded ( 5 failed automatic Talairach and were excluded). The result 
is a reconstruction of the whole brain where all brain regions are labeled. In this particular application the command "mri_cc" was used to customize CC measurement. Using the segmentation file without CC segmentation as an input, I specified the $\mathrm{CC}$ to be divided into 5 equal segments and extend segmentation 6 millimeter off the midline. The resulting file was named "cc.mgz" and was later visually inspected to ensure quality.

After all the participants were processed in this manner, a volunteer (Paula Webster) was recruited to blind the main investigator (me) from participant identity. The blinder changed the names of all the participants to a three-letter code randomly assigned and which was unknown to the investigator. A second rater (Caitlin Montgomery) was also blinded to the participants' identity, and was given both verbal and written instructions (Appendix K) to draw the CC in the Freesurfer software. Intraclass correlation coefficient (ICC) was performed between raters for the Freesurfer measurements, as well as intra-rater reliability for only the main investigator (Appendix L). Since participants from aim 1 and 2 were combined, the ICC reflects both the ICC for aims 1 and 2 . The intra-rater reliability ICC when testing all 6 MPRAGE images used in this analysis yielded a value of $0.997(p<0.001)$. The inter-rater reliability ICC under the same conditions was $0.973(p<0.001)$ for all 6 MPRAGE images. Taken together, this data suggests that the reliability between raters for this semi-automated method is outstanding.

After the blinding process, the main investigator would open up the files associated with the participants and edited the CC ROIs already produced by Freesurfer to remove errors. Errors commonly occurring in the Freesurfer segmentation ROIs include overestimation around the fornix, overestimation in the anterior regions on the more medial slices (confusion between 
$\mathrm{CC}$ and other white matter tracts in the area), and overestimation in the lateral periphery where the $\mathrm{CC}$ begins to diffuse out into general white matter. There was rarely any underestimation of the CC.

Quantification of the measurement was conducted using the mri_segstats command which takes an inputted ROI and converts it into a table which provides volumetric measurements for all of the segmented regions in the brain. These measurements are provided in cubic millimeters for the entire segmented region. Only CC_anterior, CC_midanterior, CC_central, CC_midposterior, and CC_posterior were included in the analysis. These subdivision were summed together to produce the entire CC volume. These data can be viewed in Appendix M.

There was an additional reliability measurement which was recorded as well. In order to check the quality and reliability of both the manual tracing and the semi-automated method, measurements from 6 participants were entered into SPSS and an ICC was calculated.

Measurements between the methods occurred several weeks apart and while the rater was still blinded. The ICC between the manual and semi-automated method on 6 participants using the same rater is reported to be $0.887, p<0.001$. Therefore, reliability between the two methods was very good.

Generally speaking, studies which measure CC volume will report not just the raw CC measurements, but normalize these measures to some other measurement such as total intracranial space (Giedd et al., 1996; Haut et al., 2006; Prigge et al., 2013). This method is used to try to control for other factors in CC size such as total head size, which is considered a confounding factor in many studies (including this one). In this study, because the field of view 
of the MR images centered around the throat and upper airway (see scan parameters above), often the top of the brain was not included in the images. As such, total intracranial space was not an option of normalization here.

Instead, I sought for a different method of normalization. After attempting multiple different normalization methods, I had decided that the measure represented as brain anteriorto-posterior distance multiplied by brain width was the most appropriate for normalization (see correlation table (Appendix N)). This was able to be determined because, although most of the images did not include the top of the head, there was a proportion of participants for which the top of the head was included (Two participants from Specific Aim 1, 17 participants from Specific Aim 2). The experimental normalization methods that I had been testing on these same participants were correlated with their "eTIV" (estimated total intracranial volume) measure estimated in Freesurfer as part of the recon-all command (Fischl et al., 2004). The measurements from the different normalization methods tested and descriptions are included (Appendix O). Therefore, the measure ultimately used in the CC comparison was the total volume of the ROI divided by the normalization factor (anterior-to-posterior distance multiplied by brain width). It is important to note that this normalization factor was applied both to the semi-automated Freesurfer method of CC measurements and the manual CC measurements made in AFNI.

The normalization factor itself was calculated using the AFNI program. After ACPC registration, the graphical user interface (GUI) contains the option to proceed to Talairach registration. Though the manual ROI data set was not subjected to Talairach registration, this process can be used to determine some basic brain measurements. Included in the reported 
statistics include distance from anterior-to-posterior most point in the brain, anterior commissure to posterior commissure (inter-commissural distance) and distance from left most point to right most point (brain width). It was these measurements that were multiplied and become the normalization factor. Although not ideal, in the absence of the entire brain the factor showed reasonable correlation to eTIV calculated by Freesurfer $(r=0.814, p<.001)$. CC volumes and sub-volumes were ultimately multiplied by the inverse of the normalization factor.

The neurocognitive measures used are already published, and scores were generated following the published instructions by a graduate research assistant (Pamela Tessier). Selected neurocognitive outcomes are included in Appendix $P$.

Basic demographic and infant feeding method information was obtained through survey and other study forms and entered into excel before later transfer to SPSS. Infant feeding information is included in Appendix Q. The sleep measures were acquired off-site at Dr. Clawges' pediatric sleep practice, transferred from the report into excel, and later transferred again into SPSS (Appendices R). Therefore, the information under investigation for this aim include demographic information, infant feeding methods, sleep information, CC volumetric measurements, and neurocognitive scores.

\subsection{Specific Aim 2}

\subsubsection{Description of Procedures}

In the second Specific Aim 22 healthy children were recruited to participate for MRI imaging. The parents of these children were screened and contacted on the basis of their participation in a separate sleep survey also associated with our WVU sleep laboratory. If 
parents had indicated that their child had no breathing problems during sleep or surgical removal of the tonsil and adenoids they were considered "healthy" and eligible to participate.

Surveys used to recruit parents were online surveys available for public participation (Appendix S). Advertisements for these surveys came in multiple forms, ranging from small halfsheet flyers (given to approximately 10 locals schools, 20 local daycares, 3 local businesses, pediatric wards of Ruby Memorial Hospital, etc.), to tear-off flyers (posted around the campus of WVU; in particular the life sciences building [LSB] and health sciences center [HSC] as well as Ruby Memorial Hospital), to large banners hung in prominent public areas. Electronic advertisements were administered through university news flyers, and social media was used to some extent (Facebook; Appendix T). News of the study was additionally spread through word of mouth. Participants did not necessarily have to take the survey to participate; they also had the option to call the lab, where they were screened to ensure their child did not have SDB.

The online survey itself was registered to www.surveymonkey.comand consisted of questions regarding demographics, sleep characteristics about their child, and contact information. In order to qualify for this particular study, participants had to indicate that their child was between 4 and 8 years old, and had responded that their child either "never" or "rarely" snored, as well as indicated "never" or "rarely" on other SDB related questions. A table of participant's responses is provided (Appendix U).

The procedure for intake of the participants on the day of arrival, signing consent forms and demographics paperwork, mock scanning, and actual MRI scanning was identical to the procedures used in Specific Aim 1. The consent and assent forms for this aim did vary slightly from aim 1. The only differences for this particular aim was that T2-weighted scans, although 
acquired, were not analyzed as the T-1 MPRAGE were higher quality and included the entire brain in the field of view. The T2-weighted images were useful in a separate study not directly related to this dissertation. Also, total scanning time was slightly longer for this Specific Aim due to the addition of a $\sim 7$ minute diffusion-weighted scan for DTI analysis.

As was the case in Specific Aim 1, the T1-MPRAGE scans had variable parameters for different participants, partially due to trying to find the optimal scanning parameters for imaging children. The images were generally acquired in either the axial plane $(149,234,316$, $333,483,555,682,755,863$, and 951$)$ or the sagittal plane $(119,135,296,341,454,511,654$, $694,722,749,888$, and 972 ). Axially acquired images had $0.45 \times 0.45 \times 0.9 \mathrm{~mm}^{3}$ voxels with repetition time $=1900 \mathrm{~ms}$, echo time $=2.99 \mathrm{~ms}$, flip angle $=9$, matrix $=512 \times 448$, and field of view $=201 \times 230 \mathrm{~mm}^{2}$. Sagittal acquired scans had $1 \times 1 \times 1 \mathrm{~mm}^{3}$ voxels, repetition time $=2250$ $\mathrm{ms}$, echo time $=4.63 \mathrm{~ms}$, flip angle $=9$, matrix $=256 \times 256$, and field of view $=256 \times 256 \mathrm{~mm}^{2}$. Two additional participants (296 and 888), although acquired in the sagittal plane, had the same scanning parameters as the axial images.

The diffusion-weighted images were acquired in the axial plane in $2 \times 2 \times 2 \mathrm{~mm}^{3}$ voxels. Additional parameters for the scan include repetition time $=9000-9700 \mathrm{~ms}$, echo time $=98$ $\mathrm{ms}$, flip angle $=90$, matrix $=1152 \times 1152$, and field of view $=2304 \times 2304 \mathrm{~mm}^{2}$. The DTI scan acquisition was about 7 minutes in duration.

Once all of the scan sequences were acquired the participants were taken out of the scanner. Parents were then asked to provide the child's report card, if applicable (not all of the participants were school-aged). Parents received a 150 USD gift card for their time and travel while participants were given a small toy. 


\subsubsection{Data Processing}

Unlike in aim 1, there were no additional sleep outcome variables collected in aim 2 since all participants should theoretically be healthy sleepers. All MRI variables, including both manual (Appendix V) and semi-automated CC tracing (Appendix W) with instructions for multiple raters were measured identically as in aim 1. As in the previous aim, ICC was calculated between raters for both the manual (Appendix X) and semi-automated (Appendix $M$ ) portions of the CC tracings.

In the manual tracings, using all 6 participants from both aims (4 from aim 1, 2 from aim 2 ) yielded an intra-rater ICC of $0.994(p<0.001)$ and an inter-rater ICC of $0.862(p<0.050)$ indicating good reliability. In the semi-automated tracings, using all 6 participants from both aims yields an intra-rater ICC of $0.997(p<0.001)$ and an inter-rater ICC of $0.973(p=0.001)$ as previously reported. Finally, a comparison between manual tracings and automated tracings of the same participants yielded an ICC of $0.811(p<0.001)$. In aim 1 , this same measurement was reported to be 0.887 ; this indicates pretty good reliability between the two methods in both aims.

Breastfeeding information was collected in the same manner as in aim 1, and the data for this is displayed in Appendix Y. Neurocognitive testing in this aim did not occur, but was replaced with recent academic performance using standardized report cards (Appendix Z). However, only $53 \%$ of participants with an MRI elected to provide a report card, partially due to some children really being too young to have a standardized report card, and partially due to some children having homeschooling or the parents simply declined to participate in this 
section. Report cards were generated by Monongalia county schools and are divided between reading (with several items pertaining to reading), writing, math, work habits/selfmanagement, and sometimes science and/or social science. Since science and social science are not always included, this metric was removed. Since each item can be rated needs support (NS), approaching standards (AS), meets standards (MS), or exceeds standards (ES), each item was numerically ranked based on the teacher rating ( $N S=1, A S=2, M S=3, E S=4)$. The only exception to this is the work habits/needs support section, where each item is rated by the teacher as needs support (-), developing satisfactorily $(\checkmark)$, or demonstrates strength $(+)$. This was also translated numerically like the other sections $(-=1, \checkmark=2,+=3)$. The reported score for each category (reading, writing, math, work habits/self-management) was therefore represented by the average of all items within the given section. The "Average" metric represents the average score between reading, writing, and math.

Diffusion-weighted images were processed in TRACULA (tracts constrained by underlying anatomy), a DTI reconstruction tool within the Freesurfer software package. TRACULA (Yendiki et al., 2011) uses probabilistic tractography to reconstruct the major white matter pathways of the brain, using anatomical MPRAGE scans for guidance. It is a fully automated method.

With TRACULA, a total of 18 different white matter pathways can be reconstructed; CC forceps major, CC forceps minor, bilateral anterior thalamic radius, bilateral cingulum angular bundles, bilateral cingulum cingulate gyrus, bilateral corticospinal tract, bilateral inferior longitudinal fasciculus, bilateral superior longitudinal fasciculus (temporal portion), bilateral superior longitudinal fasciculus (parietal portion), and bilateral uncinate fasciculus. 
After acquisition of the diffusion weighted images for all participants, they were visually inspected for quality before reconstructing them in TRACULA. Reconstruction in TRACULA was conducted using the suggested default settings. Outputs from TRACULA are both reported in numerical as well as visual form; reconstructed pathways can be viewed in color-coordinated representations in 3 dimensional space constrained by the MPRAGE scan. In addition, individual slices from the reconstruction will show an intensity map slice by slice for any selected pathways. All reconstructed pathways for all participants were visually inspected for quality. With very few exceptions (one or two forceps were under-reconstructed), all reconstructed pathways appeared normal.

Numerically, TRACULA will report standard measures used in all DTI analysis; fractional anisotropy (FA) scores, mean diffusivity scores, radial diffusivity, axial diffusivity, pathway length, etc. In the DTI analysis only fractional anisotropy and pathway length were ever used. Furthermore, due to the probabilistic method of reconstruction, three separate metrics are reported for FA scores; "FA_avg” (average FA value for entire tract), "FA_avg_Weight" (average FA value multiplied by the probability that voxel is included within the tract, averaged over the entire tract), and "FA_avg_Center" (average FA value over the highest probability path only). All of these metrics for FA were examined. I have not included this data in the tables or appendices, as numerous FA values for each participant for each pathway is a large quantity of data.

Finally, TRACULA also reports quantitative measures of head motion for quality assurance (Appendix AA). Head motion can be a serious problem in DTI, particularly if there are large differences between examined groups as it can not only disrupt registration of the image 
(correctable) but also decrease the intensities in the diffusion-weighted images (not correctable). It is thus recommended, at the very least, to ensure that examined groups have similar head motion metrics (Appendix $A B$ ), and that key variables are not correlated to head motion (Appendix AC), or the measures can be regressed out using regression treating them as extraneous variables (Yendiki, Koldewyn, Kakunoori, Kanwisher, \& Fischl, 2013). Surprisingly, it was found that breastfeeding duration was positively correlated with 2 motion measures (average translation and average rotation), although in the direction where one would expect motion would dampen FA scores rather than enhance them. Nevertheless, both motion measures will be controlled for in future regression analysis using covariance.

\subsection{Specific Aim 3}

\subsubsection{Description of Procedures Part 1}

The initial experiment proposed in this Specific Aim was polysomnography (PSG) of a pediatric population. Although originally planned to recruit only infants less than one year old, practicality dictated that I extend the age range to include toddlers and young children. These participants were mostly recruited via a lab survey (Appendix S), and were screened and contacted on the basis of their answers and whether they had an infant or child with SDB symptoms. Details in how these surveys were distributed (2.3.1, paragraph 2 ) and who was included for the previous aim (2.3.1, paragraph 3) are described above. Alternatively, participants were also recruited through various advertisement media put on public display such as banners, tear-off advertisements, half sheet advertisements (Appendix T) and social media. Participants recruited in this manner were screened to ensure that the child had snoring 
and/or breathing difficulties during sleep. For this particular aim, rather than recruiting only healthy children, our goal was to recruit infants and children with suspected SDB based on parental answers to our survey. Therefore, only parents that rated their child at least "frequently" on the question "How often does your child snore?" were included, or if they indicated "occasionally" with positive SDB indicators on some of the other questions, or if there was significant parental concern. A table of the parent's responses for key questions is included in Appendix AD.

Parents of the recruited participants were scheduled to come into the lab on a night of their choosing for overnight polysomnography (PSG). After giving a brief tour of the lab, parents were consented and encouraged to provide demographic (Appendix A) and infant feeding (Appendix A) information. Investigators were blinded to the infant feeding methods provided by placing the form in a sealed envelope.

After consent, parents and participants were allowed to become acclimated with the room and infants/children were provided age-appropriate toys in addition to the toys brought by the parents. Toys included various books, toy cars, plastic keys, colored rings, colored blocks, stuffed animals, slinkies, and children's shows (Elmo, Dora the explorer, etc.). Once ready, the sensors for the PSG hookup were applied to the participant. The PSG hookup in these experiments included bilateral leg electromyography (EMG), abdomen and chest respiratory plethysmography, electrocardiogram (EKG), snore microphone, pulse oximetry (Sp02) through infrared light sensor attached to the toe or finger, respiratory cannula (measuring flow pressure and $\mathrm{CO}_{2}$ concentration) and thermistor. EEG was implemented using gold-plated pediatric electrodes and the international 10-20 system. System setup included individual sensors 
plugged into the Embla N7000 amplifier and patient unit (Natus Medical Incorporated,

Pleasanton, CA, USA) which is connected to the recording computer through a communication unit. Recording software used for this study was the Embla REMbrandt Manager software version 9.1 (Natus Medical Incorporated, Pleasanton, CA, USA). Once all equipment was attached to the participant, the participant and parents were left alone in the room to undergo their normal bedroom routine until sleep. Often, a considerable amount of equipment would have to be re-applied after the participant fell asleep. The recording throughout the night also included audio and visual recording. Only a few participants were given bio-calibrations before bed, due to the young ages.

Infants and children were monitored throughout the night to ensure participant safety and quality recordings. Frequent entries to repair the PSG montage were required for most participants, often several times an hour. By the end of the night, all equipment was removed and participants and parents were thanked for their time. Parents were compensated with a 150 USD gift card.

\subsubsection{Data Processing Part 1}

Sleep scoring for each participant was done through the REMbrandt Manager software (using American Academy of Sleep Medicine (AASM) guidelines (Berry, Gamaldo, et al., 2015)). Scoring was done directly by the lab primary investigator (Hawley Montgomery-Downs) or completed by myself and then later checked by Dr. Montgomery-Downs. For the purposes of this project, sleep staging was the primary focus of the scoring but events such as apneas, hypopneas, leg movements, and arousals were also indicated. 


\subsubsection{Description of Procedures Part 2}

Due to the lack of success in recruiting a sizable SDB population, a second attempt was made to investigate the aim 3 hypothesis. In this study, I sought to use a dataset of eight to nine month old children who had undergone PSG under the suspicion of SDB, many of whom were subsequently indicated to have some level of SDB (often primary snoring, or evidence of snoring with snore microphone). Acquired approximately 11 years ago, this type of study also provided the opportunity to sample these participants again now that they have grown.

About 11 years ago (2003-2004), parents of newborn children were recruited to have their child participate in a sleep research study in several months pertaining to sleep disordered breathing in Louisville, Kentucky by Dr. Montgomery-Downs (Appendix AE). After recruitment, participants were given a survey form to fill out that included information such as name, education, general demographics, and child sleeping habits. Based on the parent's responses to these questions, the infant participants may have been recruited for overnight PSG at Kosair Children's Hospital in Louisville, Kentucky. These were standard PSG recordings that provided the usual sleep statistics and information that would be expected from a clinical service.

In the current study, I sought to send participants a new survey form (Appendix AF) to acquire infant feeding methods information; information not acquired during the original study. In addition to the standard demographic information, the same questions that were used for screening 11 years ago were asked again in this survey. In addition to the survey, participants were mailed an introductory letter reminding them of their participation, and a consent form to sign and send back along with the completed survey. Included in the envelope was a self- 
addressed letter with postage to send back to our offices. At least one in 25 respondents received a 25 dollar gift card (USD).

\subsubsection{Data Processing Part 2}

All data from the surveys were entered into excel, with the survey questions numerically coded ("never"=1, "rarely"=2, "occasionally"=3, "frequently"=4, "almost always"=5) except for question three which was scored in reverse. All of the sleep questions (ten questions) were averaged to produce the infant sleep impairment score and compared in the analyses. Data from the screening survey 11 years ago was entered in the same way. Additionally, most variables from the infant PSG were also entered into excel.

In aim 1, an SDB composite score was used to determine severity of SDB (MontgomeryDowns, O’Brien, Holbrook, \& Gozal, 2004). In this aim, we instead used the infant sleep impairment composite score calculated from answers from the survey. Although a composite score calculated from PSG-measures would obviously be preferable to survey responses, the SDB composite score is not validated for this age group (Montgomery-Downs et al., 2004). In addition, not all participants underwent overnight PSG, so utilization of the infant sleep impairment score permits a larger sample size.

\subsection{Results}

\subsection{Specific Aim 1}

\subsubsection{Hypotheses}


1a. Increased breastfeeding duration early in life will be negatively correlated with SDB severity, and both increased breastfeeding and reduced SDB severity will be correlated with increased CC volume. Ultimately, I predict that increased breastfeeding duration will be correlated with increased CC volumes through a partial mediation effect from reduced SDB.

1b. I further hypothesize that increased breastfeeding duration, reduced SDB severity, and increased CC volume will be positively correlated with neurocognitive outcomes (particularly executive function) including: inhibition control, strategic planning, working memory, and mental flexibility.

\subsubsection{Preliminary Analysis}

\subsubsection{Participant Characteristics}

Of the 27 participants who consented to participate, five did not contribute data to all experimental components: three were unsuccessful in completing the MRI portion (and therefore did not participant in neurocognitive testing and whose PSG data were unavailable), one completed all portions except the neurocognitive testing, and one participated in both the MRI portion and neurocognitive portions but dropped from PSG at the sleep clinic. Therefore, three were totally excluded and two were included in the analyses to which they contributed. For eight of the participants, the MRI scans were of insufficient quality or beyond the field of view (see section 5.1 Methods; paragraph nine) to analyze CC volumes. The remaining usable sample of MRI CC volumes was thus 16 , rather than 24 . One of these scans was initially analyzed, but was removed as an obvious outlier (13,738 cubic millimeters for CC measurement, compared to the next highest CC measurement at 9,090 cubic millimeters) most 
likely due to image quality. Final sample sizes and participation status for each component are reported in Fig 3.

Among those who consented and participated $(\mathrm{N}=24), 37.5 \%$ (nine) were female. The mean participant age was 6.8 years $(S D=1.5)$. The three who did not participate were younger than those who did $(4.5$ years; $p<0.001$ ) but otherwise did not differ from the final sample. Among those who self-reported (one missing), average maternal education was 13.9 years ( $n=23 ;$ SD =2.8). Mean self-reported income was 33,400 USD among those who reported income ( $n=16$; three additional reports of "unemployed", "not working", and one participant who claimed 500 USD annually were counted as unemployed; SD $=22,300$ ) while the median was 28,500 USD, with a range of 91,500 USD $(8,520$ to 100,000 USD). Thirteen ( $52.2 \%)$ of the participants (one report missing) were living with a parent who smoked $(n=26)$. Twenty-three participants were Caucasian (85\%), four were biracial (15\%). All participants had intact tonsils and adenoids. Thirteen (54.2\%) had been diagnosed with ADD or ADHD. Mean body mass index (BMI) measured (2 missing) at the sleep clinic was 19.1 ( $n=22 ; S D=5.9)$. General demographic information describing this sample are in Table 1.

\subsubsection{Normality and Outliers}

Descriptive statistics were calculated and data were checked for normality and outliers. Testing for normality with the Shapiro-Wilk for all variables, it was found that REM (\%TST), WJIII performance (percentile), and CC volumes (cubic millimeters) were normally distributed; however, AHI, SDB profile, BRIEF scores (percentile), Day-Night Stroop scores (raw), and PPVT scores (percentile) were not normally distributed in this sample. Outliers were tested for using 
the outlier labeling rule (Iglewicz \& Banerjee, 2001) with a g-value of 1.5 (1.5 times the interquartile range determines upper and lower limits). There were no observed outliers for REM (\%TST) and WJ-III performance. Using a g-value of 1.5, there was one outlier for normalized CC volumes, three outliers for AHI, two outliers for SDB profile, two outliers for the BRIEF, two outliers for the Day-Night Stroop, and one outlier for the PPVT. Using the less conservative g-value of three, there were no outliers for CC volumes, one outlier for $\mathrm{AHI}$, one outlier for SDB profile, one outlier for BRIEF, no outliers for Day-Night Stroop, and no outliers for the PPVT. Due to the careful and standardized measurements of all PSG and neurocognitive data, no outliers were removed from these datasets as outliers were not due to technical failure or errors in measurement. Removal of the outlier from AHI and SDB profile did not affect the results.

Data transformation was not successful in restoring normal distributions in five key variables (above) due to the presence of natural zeroes and high variance. Due to non-normal distribution, non-parametric statistical tests were applied. When comparing between groups, the Mann-Whitney $U$ hypothesis test was used in lieu of independent $t$-tests. Since the error residuals were not normally distributed for linear regression, this test was not valid. Analysis of covariance was still used for these variables despite non-normal distribution (a violation) as this particular test is somewhat robust to non-normality provided that sample sizes are approximately equal (Levy, 1980).

\subsubsection{SDB Characterization}


Using previously published methods (Montgomery-Downs et al., 2004) I calculated the SDB composite variable based on each participant's AHI, RAI, desaturation index (Desat Ind), and $\mathrm{SpO} 2$ nadir. Computation of the composite score is the sum of the scores for $\mathrm{AHI}, \mathrm{RAl}$, and the average of Desat_Ind and SpO2 nadir, divided by three (AHI and RAI are weighted more heavily). The rationale for creating this variable is that SDB often cannot be adequately represented by a single variable such as AHI (Punjabi, 2016). Therefore, the SDB profile allows a more complete quantitative picture of the individual's disorder severity. Individual SDB profile scores and contributing variables are in Appendix AG. This variable was also used to test hypothesis $1 \mathrm{~b}$.

\subsubsection{CC Corrections}

Head size was generally accounted for using the aforementioned (see 5.2 Methods; Data Processing; paragraph 16) normalization factor. CC size also changes across age, as it contains white matter that continues to mature throughout life (Tanaka-Arakawa et al., 2015). As a result, in this study, CC size was controlled for using age as a covariate, as recent research suggests that after four years of age this structure increases in size at a slow, constant and linear rate, at the very least until age eight, if not throughout life (Giedd et al., 1996, 1999; Keshavan et al., 2002; Tanaka-Arakawa et al., 2015).

\subsubsection{Aim 1 Results}

\subsubsection{Hypothesis $1 a$}


To test the first hypothesis (that increased breastfeeding duration early in life will be negatively correlated with SDB severity, and that both increased breastfeeding duration and reduced SDB severity will be positively correlated with increased CC volume), a series of correlations and linear regressions between variables were tested where appropriate.

Correlations were calculated between hypothesized variables (Breastfeeding method [coded $\{1=$ exclusive formula feeding, $2=$ both $3=$ exclusive breastfeeding $\}$ ], Breastfeeding duration [months], SDB profile, $\mathrm{AHI}, \mathrm{CC}$ volumes [normalized and raw], CC sub-volumes [anterior, central, posterior], and neurocognitive outcome variables), and significant correlations were further analyzed using multiple tests of multiple regression and controlling for potentially confounding variables (below). The AHI variable was included separately (despite that it was also part of the SDB profile) due its widespread use in characterizing SDB in the literature (Chervin et al., 2006; Guilleminault, Benbir, \& Akhtar, 2007) and ease of interpretation, although it is often not the only metric used.

If correlational analysis supported the proposed hypothesis, partial mediation testing was planned, using Preacher and Hayes' bootstrapping method (Preacher \& Hayes, 2008). However, due to a lack of statistically significant correlations between predictors and outcomes in aim 1, partial mediation testing was not warranted.

To determine whether socio-demographic measures accounted for significant variance in either predictor or dependent variables (breastfeeding duration, SDB profile, REM [\%TST] CC volumes, WJ-III performance), potentially confounding variables (age [years], sex, ADD/ADHD diagnosis, gross annual income [USD], maternal age [years], maternal education [years], and $\mathrm{BMI})$ were included in a correlation table to examine possible associations. 
Both age and ADD/ADHD diagnosis were significantly and negatively associated with both WJ-III scores and REM (\%TST). Although not significantly correlated with any variables, sex was included for its known impact on CC volumes (Sullivan, Rosenbloom, Desmond, \& Pfefferbaum, 2001) while annual income was included for the well-known socioeconomic influence on breastfeeding initiation and duration (Colen \& Ramey, 2014; McFadden \& Toole, 2006). Annual income was also negatively correlated with BRIEF scores ( $p=0.022)$. Maternal age, maternal education, and BMI were not included in regression models as they were not significantly correlated with the predictor or dependent variables. Although there could be some justification for including maternal education and BMI in the model based on previous literature (Colen \& Ramey, 2014; Gibbs \& Forste, 2014; McFadden \& Toole, 2006), in this sample only six children had a BMI greater than 20, while only two had a BMI greater than 25 suggesting obesity was limited (BMI seemed to be representative of the normal population). Since the socioeconomic influence of annual income is already included as a confounding variable, the inclusion of maternal education was deemed somewhat redundant, as it has been reported that only income contributes unique variance when looking at brain surface area and other structures (Noble et al., 2015).

The correlation matrix for relations among feeding methods, breastfeeding duration, sleep outcome variables, and CC size (Manual ROls, both MPRAGE and T2's included) is presented in Table 2. Although significant correlations were detected, none were relevant to the hypothesis (i.e. AHI was included in the SDB composite variable, so a positive correlation between these measures is unsurprising). Analyzing the anterior, central, and posterior portions of the CC separately did not reveal any additional significant correlations. Including 
other measured sleep variables (\% REM [TST], N3 [TST], REM AHI, RAI [independently], Desaturation Index [independently], SpO2 nadir [independently]) did not result in any significant correlations. Hypothesis 1a was not supported.

\subsubsection{Hypothesis $1 b$}

To test the second hypothesis that breastfeeding, reduced SDB severity, and CC volume will be positively correlated with neurocognitive outcomes (particularly executive function) such as inhibition control, strategic planning, working memory, and mental flexibility, another correlation table was generated to include the neurocognitive outcome measures. Statistically significant correlations $(p<0.05)$ were further tested with multiple regression or analysis of covariance using age, sex, and annual household income as covariates where applicable.

Longer breasting duration, but not feeding method, was positively correlated with higher scores on the Day-Night Stroop test $(r=0.422 p=0.045)$. SDB profile was positively correlated with higher scores on the PPVT $(r=0.534, p=0.011)$. An unexpected, but not entirely surprising result was that REM sleep index was significantly and positively correlated with scores on the WJIII-Exec $(r=0.573, p=0.010)$, but negatively correlated with scores on the parental completed BRIEF ( $r=-0.498, p=0.018)$. Longer breastfeeding duration approached significance for a positive correlation with higher scores on the WJIII-Exec $(r=0.442, p=0.050)$. A summary of these correlations is in Table 3.

However, correlation coefficients that do not take into consideration potentially confounding variables are insufficient. When looking at these individual relations using stepwise multiple regression with sex, age, ADD/ADHD diagnosis and annual household income 
as covariates, breastfeeding duration was no longer significantly associated with higher scores on the WJIII_Exec ( $p=0.896$; ADD/ADHD diagnosis in this model is the only significant factor), nor was REM TST a significant factor in predicting scores on the WJIII_Exec ( $p=0.764$; ADD/ADHD diagnosis was the only significant factor) or the BRIEF ( $p=0.764$; ADD/ADHD diagnosis was the only significant factor).

Linear regression is not valid for the Day Night Stroop or PPVT scores due to nonnormally distributed data, but a Mann-Whitney $U$ test revealed that there was not a statistically significant difference between exclusively formula-fed children and children with any breastfeeding (Boolean coding) in regards to the Day Night Stroop ( $p=0.088$ ). Recoding SDB_Profile score to compare children with $>=$ score of 1 with children with a score $<1$ with PPVT as the DV initially revealed a significant difference with the Mann-Whitney U test $(p=$ 0.027), but not when an analysis of covariance (ANCOVA) was conducted controlling for age, sex, ADD/ADHD diagnosis, and annual income $(p=0.316)$.

Although not statistically significant, group differences are reported in Table 4 comparing exclusively formula-fed and children with those who received any duration of breastfeeding. Alongside this is a comparison of children with an $\mathrm{AHI}$ of one or higher, versus an $\mathrm{AHI}$ of less than one in Table 5. These are displayed for the reader's convenience, despite the lack of statistically significant differences. Nevertheless, with greatly increased statistical power, there are trends that suggest certain correlations may be worthy of further investigation. Ultimately, hypothesis $1 \mathrm{~b}$ was not supported.

\subsubsection{Supplemental Analyses}


Unexpectedly (and not hypothesized), I discovered an effect of smoking within these data. Group comparisons between children with at least one parent who smokes versus those with no parents who smoke are in Table 6. Independent samples $t$-tests revealed that children with at least one parent who smoked were breastfed a shorter duration $(p=0.036)$, had less volume in the central portion of the CC (uncorrected by intracranial space normalization factor; $p=0.049)$, and had a higher score on the BRIEF $(p=0.021)$. It should be noted that when the central section of the CC was corrected by the intracranial space normalization factor, the difference between children with smoking versus non-smoking parents fell to non-significance $(p=0.143)$

Using an analysis of covariance I found that when controlling for age, sex, ADD/ADHD diagnosis, and annual income the relationship between the presence of a smoking parent and shorter breastfeeding duration was no longer significant $(p=0.087)$ but the CC differences remained statistically significant $(p=0.030)$. However, when CC was corrected by normalization it became non-significant $(p=0.124)$. The positive correlation between having a parent who smoked and higher scores on the BRIEF was no longer significant $(p=0.152)$ when controlling for covariates.

\subsection{Specific Aim 2}

\subsubsection{Hypotheses}

2a. Increased breastfeeding duration will be correlated with increased CC volume, but the magnitude of this relationship will be reduced compared to aim 1 due to the proposed (although not supported in aim 1) partial mediation effect. 
2b. In addition to CC measurements, I also measured general white matter diffusivity through DTI. I predict several positive correlations between breastfeeding duration and major white matter tracts due to a generalized increase of white matter, but particularly in the superior longitudinal fasciculus.

2c. Finally, when possible I collected academic report cards for the children who participated in this aim in lieu of neurocognitive testing as a means of measuring real-world academic success. I predict that children who were breastfed will perform better on math, reading, writing, and work habits measures than children who were not breastfed.

2d. Comparing CC volumes between aim 1 and aim 2, I expect that children with an $\mathrm{AHI}$ $>=1$ will have smaller CCs than otherwise healthy children from aim 2.

\subsubsection{Preliminary Analysis}

\subsubsection{Participant Characteristics}

Unlike in aim 1, this population of children were healthy with no symptoms of SDB. Twenty-two participants consented to be in the study. Two of the participants dropped out of the study after being unable to tolerate the MRI. One participant's scans were not analyzed due to technical error. A flow chart of participants and their status across each portion of the study are in Fig 4.

Among those who had consented and participated $(n=19), 36.8 \%$ were female (seven females). The mean participant age was 6.4 years $(S D=1.6)$. Self-reported maternal education was 17.4 years by mean $(S D=1.9)$. The mean of self-reported income (three missing) was 92,500 USD ( $n=16 ; S D=43,000$ USD) while the median was 80,000 USD, with a range of 145,000 
USD $(15,000-160,000$ USD). Three (15.8\%) of the parents had reported that at least one person living with the child smoked. All participants were Caucasian. All participants had tonsils and adenoids intact; one had a diagnosis of ADD or ADHD (5.3\%). There were no notable differences in those who consented and dropped (three) compared to those who participated. Tables of general demographical information pertaining to this sample are in Table 7.

\subsubsection{Normality and Outliers}

Descriptive statistics were analyzed and normality and outliers were examined. Using Shapiro-Wilk test of normality for all variables, it was found that all CC measurements, both normalized and non-normalized, were normally distributed. Under the criterion of the outlier labeling rule (greater than 1.5 times the interquartile range from either the $1^{\text {st }}$ or $3^{\text {rd }}$ quartile), there were no outliers in this dataset.

For the DTI data and for all white matter paths examined (CC forceps major FA, CC forceps minor FA, Left angular bundle FA, Left superior longitudinal fasciculus FA) there was a normal distribution using the Shapiro-Wilk test of normality and no data points met outlier criteria.

For the report cards, the calculated average scores (see 6.2; Data Processing; paragraph 3) were normally distributed, with a single outlier. The work habits/self-management scores were not normally distributed, but contained no outliers. Due to the standardized nature of these report cards (standardized across Monongahela County), the outlier in the average scores was not removed. 
In order to compare groups between exclusively breastfed and not exclusively breastfed for the work habits scores, the non-parametric Mann-Whitney $U$ statistic was used in place of the independent $t$-test due to non-normal distribution. Despite non-normal distribution being a violation of the analysis of covariance, this test was still used to control for covariates, as literature suggests the ANCOVA is robust in regards to normality of distribution when sample sizes are similar (Levy, 1980).

\subsubsection{Aim 2 Results}

\subsubsection{Hypothesis 2a}

In order to test the hypothesis that breastfeeding duration and CC volumes and subvolumes (anterior, anterior_central, central, posterior_central, posterior) were correlated, a series of correlation tables between variables were conducted. Due to the lack of statistically significant relationships $(p<0.05)$ between breastfeeding measures and CC volumes, multiple regression was not subsequently warranted.

There were no statistically significant correlations between breastfeeding duration and any CC measures, in whole or in part. Additionally, group differences between exclusively breastfed and exclusively formula-fed using independent $t$-tests were not significant. There were no relevant trends to report. Parental smoking was not correlated with any portion of the CC volume. Hypothesis 2a was not supported.

\subsubsection{Hypothesis $2 b$}


Examining potential relationships between fractional anisotropy (FA) scores of major white matter tracts (18 tracts total as provided by TRACULA) and breastfeeding metrics, I also generated a correlation table between these variables. Statistically significant correlations $(p<$ 0.05) were analyzed further using stepwise linear regression.

To determine whether socio-demographic measures accounted for significant variance in either predictors or dependent variables (breastfeeding duration, CC volumes, report card metrics [average score; work habits score], DTI metrics [FA for Left SLFT; Left Angular Gyrus]), potentially confounding variables (age [years], sex, gross annual income [USD], maternal age [years], maternal education [years], and parental smoking) were included in a correlation table to examine possible relationships with the predictors or dependents. Age was positively correlated with CC volume (Freesurfer; raw). Although there were no other significant correlations between any of the other variables tested, age, sex and annual income were still used as covariates due to the aforementioned associations established in the literature (Colen \& Ramey, 2014; McFadden \& Toole, 2006; Sullivan et al., 2001).

Parental smoking was also included as a covariate due to the potentially interesting finding in aim 1 between smoking and breastfeeding duration. Maternal age and maternal education were not included in subsequent analyses because they did not correlate with predictor or outcome variables. As in aim 1, maternal education was also excluded as recent evidence suggests that only income introduces unique variance when looking at brain structures (Noble et al., 2015). In order to correct for head motion in the diffusion weighted images, average translation and average rotation (metrics reported by TRACULA) were used as covariates and controlled for in those specific analyses. 
Stepwise linear regression controlling for sex, age, annual income, parental smoking, average translation, and average rotation revealed a significant positive relation between breastfeeding duration and the FA scores for the left cingulum angular bundle (LAB; $p=0.034$; $R^{2}=0.301$; Fig 5), where breastfeeding duration was the only significant predictor. Additional linear regressions controlling for the same variables indicated that breastfeeding duration was positively correlated with FA scores ("center"; average from only the most probable pathway [most conservative estimate]) for the left superior longitudinal fasciculus (LSLF; temporal portion; $p=0.019 ; \mathrm{R}^{2}=0.354 ;$ Fig 5 ), where breastfeeding duration was the only significant predictor, but not the left superior LSLF parietal portion when motion metrics (average translation; average rotation) were included in the model. Only when both motion measures were omitted from the model (and parental smoking) did breastfeeding become significant ( $p=$ 0.033), perhaps suggestive that the LSLF parietal portion finding could be an artifact of motion. Nevertheless, positive correlations between breastfeeding duration and FA scores for the LAB and LSLF temporal portion survived correction for covariates (Fig 5).

An independent $t$-test between exclusively breastfed children and children who were not exclusively breastfed as infants also indicated a significant difference in center FA scores for the LSLF temporal portion $(p=0.002)$. An analysis of covariance revealed that this difference was still statistically significant after correction for sex, age, annual income, parental smoking, average translation, and average rotation, with exclusively breastfed children having higher FA scores $(p=0.034)$. Only breastfeeding duration was a significant predictor. Although results must be interpreted with caution because of changes with motion covariates, hypothesis $2 \mathrm{~b}$ was supported. 


\subsubsection{Hypothesis 2c}

Testing the potential role of exclusive breastfeeding vs. not exclusive breastfeeding on academic success, various academic metrics were tested for correlation compared to breastfeeding duration, exclusive breastfeeding, and exclusive formula-feeding. Statistically significant correlations were further analyzed with multiple regression or $t$-tests and analysis of covariance where appropriate.

A Mann-Whitney $U$ test between children exclusively breastfed compared to not exclusively breastfed indicates that exclusively breastfed children were trending with higher "work habits" scores by report card than children who were not $(p=0.068)$. The ANCOVA using age, sex, annual household income, and parental smoking as covariates revealed that there were statistically significant differences between the two feeding groups $(p=0.028)$, with exclusively breastfed children scoring higher, although annual income was a statistically significant covariate $(p=0.036)$. It is noteworthy that sample sizes were not equal ( $n=3$ vs. $n=7)$. There is very weak support for elements of hypothesis $2 c$.

\subsubsection{Hypothesis $2 \mathrm{~d}$}

Here, I compared the CC volumes (manual ROI in T1 and Freesurfer method) between children in aim 1 with an $\mathrm{AHI}$ rating greater than or equal to one and otherwise healthy children from aim 2. In addition to this, despite caveats described below, a comparison also was made in which the T2-weighted images from aim 1 were also included in the analysis. The addition of the T2 images increases statistical power between the two groups, but also introduces images 
to which the raters were un-blinded (T2-weighted images were only present in the SDB grouptherefore raters would be aware that these CCs were associated with participants not in the healthy group).

Volumes and sub-volumes were compared between groups (exclusive breastfeeding vs. any formula, or exclusive formula-feeding vs. any breastfeeding) using independent $t$-tests.

Comparison of data between Specific Aim1 and 2 (SDB population vs. healthy population) were also conducted (Table 8). Although there were no significant differences, the comparison between uncorrected CC central portions (through Freesurfer) approached trend level ( $p=0.100$; corrected CC Central=0.116).

Including the T2 manual ROIs, an independent $t$-test between groups indicated that there was a significant difference in total, anterior, central, and posterior $\mathrm{CC}$ size $(p=0.002, p=$ $0.009, p=0.002, p=0.003$, respectively), with the healthy group having significantly larger structures (7832 $\mathrm{mm}^{3}$ compared to $6481 \mathrm{~mm}^{3}$ for total CC size; hedges' $\mathrm{g}=1.32$ ). However, when accounting for head size, none of these differences remained statistically significant $(p=$ 0.155; total CC volumes). Hypothesis $2 \mathrm{~d}$ was not supported.

\subsubsection{Supplementary Analyses}

There were only two children with parents who smoked in this aim, yet a significant difference was found in FA scores ("Center" portion) for CC forceps minor using an independent $t$-test $(p=0.003)$. Although this result must be interpreted with caution, stepwise multiple regression with six (parental smoking is the predictor; breastfeeding duration now becomes a 
control) control variables produced a model with smoke exposure as the only significant predictor ( $p=0.006 ; \mathrm{R}^{2}=0.485$; smoke exposure group had significantly reduced FA scores).

\subsection{Specific Aim 3}

\subsubsection{Hypotheses}

3a. Increased breastfeeding duration and reduced SDB will both be correlated with an increased percentage of REM sleep, and in cases of SDB this REM sleep will be more fragmented.

Due to the lack of SDB in the recruited sample in Specific Aim 3, a new solution was needed to fully test the hypothesis. As mentioned in the methods, this new solution involved recruiting a pediatric population who had overnight PSG at 8 months old roughly $10-12$ years prior. Breastfeeding information was acquired by mailed survey, while SDB metrics and REM sleep information was already on hand from the PSG data. The hypothesis for this new population is referred from now on as hypothesis $3 b$.

3b. Investigating PSG data and breastfeeding information acquired by survey, I hypothesize that breastfeeding duration will be positively correlated with percentage of REM sleep, as well as negatively correlated with SDB metrics. SDB will be negatively correlated with REM sleep.

\subsubsection{Preliminary Analysis}

\subsubsection{Participant Characteristics}

\subsection{Hypothesis 3a Participants}


Among the 19 participants who consented, two were unable to sleep during the night (and were subsequently excluded), three slept for some length of time but did not reach all necessary stages (and were subsequently excluded), and 14 slept an adequate amount to be included in analysis. Summary statistics for the participants is reported in Table 9 for demographics and infant feeding methods and Table 10 for sleep statistics.

Out of the 14 with available data who were included in analysis, 57\% (eight) were female. The average age was 34.8 months (2.9 years). However, the age distribution is roughly bi-modal, comprised of a younger group (average age: 15.0 months [five participants]) and an older group (average age: 45.2 months [nine participants]). All participants were under five years old.

Results from this experiment revealed that, according to PSG metrics and physician diagnosis, none of the participants actually had SDB. Therefore, all information pertaining to apneas, hypopneas, snoring arousals, etc. were excluded from analysis. Also, since the presence of SDB was a critical component to what was proposed in this dissertation, the finding of no SDB necessitated a different population to further investigate this Specific Aim.

\subsection{Hypothesis 3b Participants}

The original study enrolled 66 participants, although not all of these participants actually underwent PSG. All participants did, however, complete the screening questionnaire. Of the original 66 , thirty-two participants ( $48 \%)$ responded to our survey. Twenty-three ( $72 \%)$ of these respondents had children who had undergone completed overnight PSGs. Among the 50 
who had undergone completed overnight PSGs, twenty-three (46\%) responded. A flow chart of participation is provided in Fig 6.

Two of the 23 PSG participants did not have any information pertaining to respiratory arousals, and as such variables such as respiratory arousal index during active sleep and snoring arousal index during active sleep could not be ascertained. Furthermore, when determining the percentage of participants who were diagnosed with "primary snoring" by the interpreting physician (Dr. Gozal), two were excluded due to the presence of upper airway resistance syndrome in one participant and central sleep apnea in another.

For all 32 participants, thirteen (40.6\%) were female. Median income was 90,000 USD, with a range of 990,000 USD (10,000-1,000,000 USD). A table of demographics and infant feeding methods per participant is provided in Table 11. A table of summary sleep information is provided in Table 12, while sleep questionnaire data are provided in Appendix AH.

\subsubsection{Hypothesis 2a Normality and Outliers}

Measured sleep stages (as \% of TST) and wake after sleep onset (WASO; \%SPT) were found to be normally distributed using the Shapiro-Wilk test. There were no outliers for REM sleep or N2 sleep. There were two outliers detected when looking at N3 sleep, using the outlier labeling rule of these points being outside 1.5 times the interquartile range. There was one outlier detected for WASO. Number of awakening was not found to be normally distributed, with one outlier. Despite the presence of outliers, none were removed due to standardized procedures, eliminating the possibility of outliers due to measurement error. None of these 
outliers were extreme, as when the criteria for outliers was increased to two times the interquartile range, no outliers remained.

In order to account for non-normal distribution in awakenings, differences between groups were compared using the Mann-Whitney $U$ hypothesis test as opposed to independent $t$-test. Significant differences were further tested using an analysis of covariance controlling for sex. A non-normal distribution is not a violation of this test, provided that the residuals are normally distributed. Using the Shapiro-Wilk test, it was found that the residuals were normally distributed ( $p=0.852)$, permitting the use of this test.

\subsubsection{Hypothesis $2 b$ Normality and Outliers}

Using the Shapiro-Wilk test for normal distribution, it was found that REM sleep (\%TST) and WASO (\%SPT) were normally distributed, but SDB-related variables such as active RAI, snore arousals during active sleep, and infant sleep impairment score by survey were not. One outlier was detected in the REM sleep dataset at 1.5 times the interquartile range (but disappeared at two times interquartile range), three for active RAI, two for snore arousals during active, and two for the infant sleep impairment score. Due to the relatively standardized methods of PSG, the restricted scale of the surveys, and the absence of any extreme outliers, no outliers were removed from the dataset.

Violations in normal distribution in the SDB variables were planned to be accommodated by using Mann-Whitney $U$ hypothesis testing as opposed to independent $t$ tests. When using linear regression and analysis of covariance, non-normal distribution is acceptable provided that the error residuals are normally distributed. Using the Shapiro-Wilk 
test for normal distribution, it was determined that none of the error residuals were normally distributed.

In order to correct for non-normally distributed data, infant sleep impairment scores were transformed by taking the cube root of the data. After transformation, a Shapiro-Wilk test confirmed normal distribution. Due to the presence of natural zeroes, snore arousals during active and RAI active could not be transformed into a normal distribution. Non-parametric testing such as the Mann-Whitney $U$ test was used for these variables, but linear regression is not valid. Analysis of covariance was still performed on non-normal distribution due to robustness of normality violations when group sizes are approximately equal (Levy, 1980).

\subsubsection{Aim 3a Results}

\subsubsection{Hypothesis 3a}

Hypothesized variables were first screened for significant correlations, and significant correlations were further analyzed by multiple regression, independent $t$-test, and analysis of covariance where appropriate.

Potentially confounding variables (age [years], sex, maternal education [years]) used as covariates were determined by using a correlations table; variables accounting for significant variance in either predictors or dependent variables were used as covariates (breastfeeding duration, REM [\%TST], N3 [\%TST], N2 [\%TST], Awakenings, and WASO [\%SPT]).

Age was not found to be significantly correlated with any of the predictors or dependent variables, and thus was not used. Sex was significantly correlated with WASO, with males having more WASO throughout the night than females. Maternal education was also positively 
correlated with breastfeeding duration. Sex and maternal education were used as covariates in subsequent analyses.

A simple correlation table revealed no significant correlations, but there was a negative trend between breastfeeding duration and number of awakenings $(r=-0.506 ; p=0.065)$ and wake after sleep onset (WASO [\%SPT]; $r=-0.501 ; p=0.068$ ). A partial correlation controlling for the effect of sex and maternal education resulted in the removal of the trend in the case of WASO ( $p=0.290)$, but resulted in statistical significance between breastfeeding duration and awakenings $(p=0.015)$. A simple independent $t$-test revealed that children who were breastfed for a duration greater than five months had significantly less WASO (\%SPT; $p=0.037$ ) than children who were not, despite similar ages between groups (greater than five $=31.9$ months, five or less $=37.7$ months; $p=0.530$ ). Also, children with greater than five months' breastfeeding duration had significantly fewer awakenings throughout the night than their counterparts breastfed for a shorter duration (using the Mann-Whitney $U$ test, $p=0.032$ ). An analysis of covariance controlling for sex and maternal education indicates that children with greater than five months of breastfeeding did not have significantly less WASO (\%SPT; $p=$ $0.370)$ or significantly fewer awakenings $(p=0.062)$ than those children not breastfed for at least five months. Hypothesis 3a was ultimately not supported.

\subsubsection{Hypothesis $3 b$}

Hypothesized variables were first screened for significant correlations, and significant correlations were further analyzed by multiple regression, independent $t$-test, and analysis of covariance where appropriate. 
To determine whether socio-demographic measures accounted for significant variance in either predictors or dependent variables (breastfeeding duration, REM sleep [\%TST], WASO [\%SPT], active RAI, snore arousals during active, and infant sleep score), potentially confounding variables (sex, gross annual income [USD], maternal age [years], maternal education [years], and parental smoking) were included in a correlation table to examine possible relationships with the predictors or dependents.

Maternal age, sex, and gross annual income were not found to be correlated with any of the predictors or dependent variables and were excluded from analysis. Maternal education, which was positively correlated with breastfeeding duration $(p=0.013)$ and strongly negatively correlated with the infant sleep impairment score (transformed; $p=0.003$ ) was found to be the more relevant socioeconomic control. All participants were roughly the same age in this sample (8 months old). Parental smoking was strongly negatively correlated with breastfeeding duration $(p=0.003)$.

Stepwise linear regression, controlling for maternal education and smoking for either parent, revealed a significant negative relationship between breastfeeding duration and REM sleep (\%TST; $p=0.006 ; \mathrm{R}^{2}=0.325 ;$ Fig 7 ). Concerning the questionnaire, there was a significant negative relationship between breastfeeding duration and comprehensive infant sleep impairment score when controlling for parental smoking (transformed; $p=0.016 ; R^{2}=0.171$ ), but not when maternal education was added into the model ( $p=0.181 ;$ Fig 8$)$. There was no significant correlation between transformed sleep impairment score and REM sleep (\%TST; $p=$ 0.205) using a partial correlation to control for maternal education and parental smoking. There was a trend when covariates were not controlled $(p=0.070)$. 
Also conducted were independent $t$-tests and Mann-Whitney $U$ tests between exclusive breastfeeding vs. any formula-feeding, and exclusive formula-feeding vs. any breastfeeding. Demographics and significant differences are presented in Tables 13 and 14. Summarizing, those who were exclusively breastfed had significantly less active (REM) sleep (\%TST) than those with any formula-feeding $(p=0.039)$. Additionally, the exclusive breastfeeding group had significantly reduced transformed comprehensive infant sleep impairment scores than infants not exclusively breastfed $(p=0.002)$. Finally, using the Mann-Whitney $\mathrm{U}$, it was found that exclusively formula-fed infants had significantly higher active (REM) respiratory arousal indices $(p=0.009)$ and had higher snoring arousal index during active $(p=0.019)$ than infants with any breastfeeding.

An analysis of covariance reveals that there is still a significant difference between exclusive breastfeeding and any formula with both REM sleep as the dependent variable $(p=$ $0.021)$, and transformed infant sleep impairment scores $(p=0.041)$. An analysis of covariance further revealed that there was no statistically significant difference between exclusively formula-fed infants and non-exclusively formula fed with active respiratory arousal indices $(p=$ 0.319) when parental smoking and maternal education was controlled, nor with regard to snoring arousal index during active $(p=0.479)$.

Despite the significant correlation between breastfeeding duration and REM (\% TST), another significance test was conducted since those children who breastfed greater than 8-9 months would have been breastfed for 8-9 months only at the time of the measurements. In order to address this, an analysis of covariance controlling for maternal education and parental smoking was conducted between those infants that were breastfed greater than 10 months 
(breastfeeding at the time of the study) as opposed to infants breastfed 10 or less than 10 months (Either no longer breastfed or likely weaning from breastfeeding). This difference was revealed to be significant $(p=0.049)$. Additionally, comparing greater than 6 months breastfeeding to 6 months or less revealed a statistical trend $(p=0.058)$.

Using Pearson's Chi-Square test, it was found that significantly more exclusively formula-fed infants snored during the night as indicated by PSG (6/6) compared to their peers with any breastfeeding $(6 / 17 ; p=0.006)$. Furthermore, exclusively formula-fed infants were statistically more likely to be diagnosed with "primary snoring" (4/5) by the interpreting physician (David Gozal, M.D.) than infants with any breastfeeding $(4 / 16 ; p=0.027$; two excluded due to diagnosis of upper airway resistance syndrome (one month breastfed) and central sleep apnea (exclusive formula-feeding).

Due to the fact that the above Chi-Square tests yielded low expected counts in several cells ( 2 were less than 5 in both instances), Fisher's exact test was also applied. This test found that there was a significant difference between exclusively formula-fed infants and infants with any breastfeeding for both snoring on the PSG $(p=0.014)$ and diagnosis of "primary snoring" ( $p$ $=0.047)$.

Comparing infants who were designated as having "primary snoring" compared to children who were not using Mann-Whitney $\mathrm{U}$, those with primary snoring had a significantly higher snore arousal index during active sleep $(p<0.001)$, significantly higher active sleep RAI ( $p$ $=0.007)$, but no significant difference in active sleep using independent $t$-test $(\% \mathrm{TST} ; p=0.151)$. Despite non-significance, active sleep (\%TST) was not in the hypothesized direction (those with 
primary snoring had non-significantly higher active sleep) which is consistent with the finding regarding breastfeeding duration.

An analysis of covariance controlling for maternal education and parental smoking indicated that infants designated as having "primary snoring" had significantly higher snore arousal index during active sleep $(p=0.011)$, but not significantly higher RAl during active $(p=$ $0.227)$.

Finally, as expected quiet sleep (N2 and N3 sleep combined) was not correlated with breastfeeding duration $(p=0.531)$, unlike active sleep. As a lower proportion of REM sleep is often interpreted as more mature sleep at this age (ontogenetic hypothesis; Roffwarg et al. 1966) we sought to investigate the proportion of REM sleep compared to quiet sleep as well. Creating a new variable in SPSS, we took active sleep (\%TST) and divided by quiet sleep (\%TST) to produce a ratio of active sleep compared to quiet (active sleep ratio). An initial correlation revealed a strong negative trend between breastfeeding duration and active sleep ratio ( $p=$ 0.053). Using a partial correlation controlling for maternal education and parental smoking, it was found that breastfeeding duration and ratio of active sleep compared to quiet sleep were not significantly negatively correlated $(p=0.192)$. There is fairly strong evidence to reject hypothesis $3 \mathrm{~b}$ in favor of the opposite direction hypothesized regarding breastfeeding duration and REM sleep. There is weak to moderate evidence to support the hypothesis that breastfeeding duration is negatively correlated with SDB metrics.

\subsection{Discussion}

\subsection{Specific Aim 1}




\subsubsection{Hypothesis $1 \mathrm{a}$}

CC volume was not significantly correlated with breastfeeding duration or SDB as hypothesized. There were no significant correlations found between infant feeding methods, SDB outcomes, sleep outcomes, or CC volume. Hypothesis 1a was not supported despite evidence in previous literature which suggests that breastfeeding duration might be correlated with white matter and brain structure changes (Deoni et al., 2013; Isaacs et al., 2010).

Despite the lack of statistical significance, results presented in Table 5 and 6 (particularly 6) indicate, through moderate to large effect sizes, that a subtle correlation between SDB and CC volume may exist if more statistical power could be achieved. Many of the effects are in the predicted direction (formula-fed children had higher AHI's, both formula-fed children and children with $\mathrm{AHI}>1$ had smaller CCs) but small sample sizes and large standard deviations contributed to a lack of statistical significance. Indeed, using the free software program G* power (version 3.1.9.2) which calculates study power and can be adapted to statistically determine necessary sample size (Faul, Erdfelder, Lang, \& Buchner, 2007) it was found that a total sample size of 46 would be required to detect a significant difference between SDB groups with the current group sample size ratio. Using the same program, it was found that it would take a total sample size of 336 to find a significant difference between exclusively formula-fed and children with any breastfeeding. Finally, $\mathrm{G}^{*}$ power reported that to find a significant correlation between CC volume and breastfeeding duration, a total sample size of 509 would be required. Therefore, at least in this first aim, the collected data suggests that further investigation between breastfeeding duration and CC volumes may be impractical, but with an increased sample size CC volume and AHI may be worth additional investigation. 


\subsubsection{Hypothesis $1 \mathrm{~b}$}

Breastfeeding duration, SDB severity, and CC volumes were not found to be significantly correlated with neurocognitive performance as hypothesized. Before controlling for covariates, it seemed there were correlations between REM sleep (\%TST; also breastfeeding duration) and some of the neurocognitive tests, but this turned out to be confounded by ADHD diagnosis, and an artifact of age (WJII-Exec) and income (BRIEF). Interestingly, annual income level was negatively correlated with the parentally scored BRIEF $(p=0.022)$, perhaps indicative that children with higher income parents had either more executive impairment, had parents with significantly higher expectations of executive function than parents with lower incomes, or that parents with higher incomes were overall more concerned than lower income parents regarding executive function, resulting in lower scoring when rating their child. These particular effects have not been investigated before, at least to my knowledge.

The SDB profile variable, which factored in multiple SDB variables, was positively correlated with higher PPVT scores before controlling for covariates. However, from a theoretical perspective, this is an unanticipated direction. Children with more impaired sleep and higher SDB severity would be expected to score lower, not higher on the PPVT (a test of vocabulary and verbal ability). This expectation is based on the observation that children with SDB have greater verbal impairment than healthy children (Bourke et al., 2011a; Halbower et al., 2006; Hunter et al., 2016; Kohler et al., 2009). However, when means were compared between children with an SDB score $>=1$ and an SDB score $<1$ it was found there was not a significant difference when using an ANCOVA, suggestive that covariates may help explain this 
finding (age was the strongest covariate, but not statistically significant at $p=0.199$ ) in this particular study. Previous studies which have found an effect have likewise controlled for this covariates when necessary (Bourke et al., 2011a; Halbower et al., 2006; Kohler et al., 2009).

The unexpected finding between children with non-smoking parents and those with at least 1 smoking parent showed significant differences in breastfeeding duration, central CC size (uncorrected), and BRIEF scores, albeit with no correction for covariates. None were significant when covariates were applied. As in the case with income, the BRIEF effect was in the opposite predicted direction; children with parents who smoked were rated significantly better in executive function than children whose parents did not smoke by said parents on the instrument. This finding is unexpected in that second hand smoke exposure has been linked to reduced academic and neurocognitive performance in children and adolescents (Chen, Clifford, Lang, \& Anstey, 2013). I suspect this may be a difference in parent expectations, and perhaps not in executive functioning, as there were no significant differences in any other neurocognitive measures. Parental expectations and ratings of executive function can often differ from actual performance by the child (Mares, McLuckie, Schwartz, \& Saini, 2007), as parental report can often be influenced by other factors (Joyner, Silver, \& Stavinoha, 2009). It has also been suggested that rater-based measures of executive function (such as the BRIEF) may measure independent cognitive constructs compared to performance-based measures (all the other neurocognitive measures in this study [Mahone et al., 2002; Toplak, West, \& Stanovich, 2013]).

Children with at least one smoking parent had smaller raw central portions of the CC than children with nonsmoking parents $(p=0.030)$. However, when these portions were 
corrected by the normalization factor there was no longer a significant difference between groups. The normalization factor is nonetheless necessary to include because it helps to correct for differences in head size (more specifically, intracranial volume), a standard correction in CC research studies (Haut et al., 2006; Prigge et al., 2013; Sullivan et al., 2001) . Nevertheless, this very preliminary evidence, I believe, justified further investigation of smoking on brain structures in later aims in this dissertation. However, at this stage a correlation between smoking and CC volumes cannot be established given the lack of statistical significance after normalization, and the lack of significance for breastfeeding duration and BRIEF scores once covariates were corrected by the ANCOVA.

\subsection{Specific Aim 2}

\subsubsection{Hypothesis $2 a$}

Breastfeeding duration was not found to be correlated with CC volumes. This was true using either manual or semi-automated tracings and infant feeding methods. Hypothesis $2 a$ was not supported.

Although it is unclear exactly as to why hypothesis $2 a$, or hypothesis $1 a$, was not supported, there are two reasonable conclusions. First, there could simply be no correlation between breastfeeding duration and CC volume. Although originally hypothesized that breastfeeding would have a global white matter effect, it may be that this is simply not the case, and that changes in white matter are actually localized to specific tracts which does not include the CC. Secondly, it may be that there was simply insufficient power in either aim. Despite findings in the anticipated direction with reasonably large effect sizes, there was still no 
statistically significant results. It may be that there is such a large variation in CC size in the population that a sample size of $15-20$ is simply not sufficient. Using G*Power, it was calculated that to be able to detect a difference in CC volume in aim 2 between exclusively formula-fed children and children with any breastfeeding, a sample size of 424 would be required assuming the same proportion of group sizes. Furthermore, in order to find a correlation between breastfeeding duration and CC volumes, a sample size of 484 would be required. Therefore further investigation of breastfeeding duration and CC sizes would likely be impractical in healthy children according to the results of aim 2 .

Continuing the power analysis for the comparisons between the healthy children in aim 2 and the SDB children in aim 1, G*Power suggests that a sample size of 46 would be required to detect a significant difference between groups using the semi-automated method Freesurfer. Since a significant difference between groups was already detected via AFNI, a power analysis was conducted to determine the necessary sample size if raw scores were corrected my normalization; in this instance a sample size of 56 would be required. Therefore, assuming proper blinding procedures were used in the future, comparison between SDB groups could be fruitful given sufficient sample size, in agreement with the power findings in aim 1.

\subsubsection{Hypothesis $2 b$}

Hypothesis $2 \mathrm{~b}$ did have some support. Analyzing 18 unique white matter tracts, only the left-lateralized superior longitudinal fasciculus (LSLF) "center" (most probable pathway only) FA scores and both standard and weighted FA scores for the left angular bundle positively correlated with breastfeeding duration after correcting for covariates. The superior longitudinal 
fasciculus, particularly the left SLF, has been implicated in working spatial memory (Karlsgodt et al., 2008; Vestergaard et al., 2011), as well as language and reading functioning (Kamali, Flanders, Brody, Hunter, \& Hasan, 2014; Nagae et al., 2012). It is thought that the left angular bundle may play a role in memory, particularly verbal memory (Ezzati, Katz, Lipton, Zimmerman, \& Lipton, 2016). Altogether, these observations are consistent with the findings in other studies that those infants who were breastfed for a longer duration scored better on neurocognitive scores as children and adolescents, particularly IQ, than their less breastfed counterparts (Isaacs et al., 2010; Kramer et al., 2008; McCrory \& Murray, 2013). If the observation in the current study that the left SLF is structurally reduced (the general interpretation from reduced FA scores) in children with less breastfeeding can be extrapolated to a larger population, then it may help to explain these cognitive differences from an anatomical perspective.

Another finding from the DTI data indicates that parental smoking may have a negative impact on CC connectivity (forceps minor "center") as indicated by FA score. This finding was statistically significant, but should be replicated due to the very low sample size. Nevertheless, in conjunction with the finding in aim 1 that parental smoking is negatively correlated with central CC size (uncorrected) this finding is suggestive of an overall negative CC impact from smoking, at least in this very small sample. Other studies investigating both smoke exposure and DTI in the CC in pediatrics are sparse, but one such study found that both prenatal smoke exposure and active participant smoking in adolescents was actually positively correlated with frontal and CC FA scores, particularly in the genu of the CC (Jacobsen et al., 2007). In contrast, another study looking at the impact of prenatal maternal smoking on later CC volumes in 
adolescents found that CC volumes were smaller in adolescents with prenatal exposure compared to adolescents without, but only in females. This effect was limited to the central and posterior portions, but not the anterior portion (Paus et al., 2008). More investigation into this area is needed.

Results from DTI suggest that there may be differences in major white matter tracts other than the $\mathrm{CC}$ when it comes to breastfeeding. These results, combined with the lack of results from $\mathrm{CC}$ volumes in both aims, may imply a left-lateralized effect in major white matter pathways rather than a more global, inter-hemispheric effect. Additional research is needed on this topic, both with regards to breastfeeding duration and SDB.

\subsubsection{Hypothesis $2 \mathrm{c}$}

No significant correlations were found between reading, writing, math, or the average of these scores on standardized report cards and breastfeeding duration. Children who were exclusively breastfed had better "work habits/self-management" scores than children not exclusively breastfed after controlling for covariates with ANCOVA, which may be indicative of better behavior in this small sample size.

\subsubsection{Hypothesis $2 d$}

Results in this section are somewhat confounded in that including both T2-weighted images and MPRAGES for comparison between aims introduces blinding issues; T2-weighted images were only used in the first aim. Limitations with blinding aside, manually traced raw CC volumes in aim 2 were significantly larger than the ones in aim1, initially suggesting healthy 
children may have more developed CCs than children with SDB (with an AHI greater than 1). However, when comparing the normalized volumes, none of these comparisons were significant. Ultimately, as with hypothesis $1 \mathrm{a}$ and $2 \mathrm{a}$, hypothesis $2 \mathrm{~d}$ was not supported.

\subsection{Specific Aim 3}

\subsubsection{Hypothesis 3a}

Investigating the putative link between breastfeeding, SDB, and REM sleep in the first part of this aim proved impossible due to a lack of SDB in the recruited sample. Instead, the second part of this aim was focused on infant sleep and PSG using retrospective surveys sent out to parents of participants who were already studied. Ultimately, in the first part, there were no significant findings after controlling for covariates. Although approaching significance only, the negative correlation between breastfeeding duration and awakenings, as well as between breastfeeding duration and WASO, may indicate more consolidated sleep (less fragmented) in general the longer one is breastfed, if we are willing to interpret this finding. There was not a statistically significant difference between children with more than 5 months' breastfed duration and children with less than 5 months' duration in WASO (\%SPT) despite similar ages; the difference was only significant if sex and maternal education were not controlled. It should also be noted that age may be a serious confounder in this experiment, as infants as young as 9 months old and children as old as 4.8 years were all included in the analysis. Considering these issues and difficulty in recruitment of PSG-validated SDB, the experiment in Specific Aim 2b became necessary.

\subsubsection{Hypothesis $3 b$}


Breastfeeding duration, as measured by number of months breastfed, is negatively correlated with the percentage of active (REM) sleep. This is interesting in that it is in the direct opposite of what was hypothesized. In order to make sense of this result, I refer the reader to the chi-squared results for interpretation. The results from PSG indicate that exclusively formula-fed infants are more likely to snore compared to infants with any breastfeeding, and the same was true when comparing diagnosis of "primary snoring". These preliminary results may suggest that breastfeeding is associated with less snoring (cardinal symptom of SDB), thus less disruption and fragmentation of certain sleep stages, particularly REM sleep. Sleep fragmentation is a known effect of SDB (Daurat et al., 2008; Gozal \& Pope Jr., 2001; Gozal, Wang, \& Pope Jr., 2001; Punjabi et al., 1999, 2002; Short \& Banks, 2014). This may help explain the percentage of REM sleep result; infants with more breastfeeding have more consolidated, less fragmented REM sleep, hence less REM sleep may be sufficient. Infants with less breastfeeding, however, having more fragmentation of REM sleep may need more REM sleep to accommodate for fragmentation, as shown by the REM rebound effect in adult populations (Beersma, Dijk, Blok, \& Everhardus, 1990), although this has been demonstrated in the context of deprivation rather than fragmentation.

Additionally, healthy infants and children with less REM sleep overall could be considered to have more mature sleep (Roffwarg et al., 1966) because REM sleep decreases rapidly in developmentally normal humans as a function of age. It may be that infants with greater breastfeeding somehow have more developmental advancement in regards to sleep than those with less breastfeeding. The precise mechanism for such an effect would need additional investigation, but nutritional or REM consolidation mechanisms are plausible. 
The association between exclusive breastfeeding and lower sleep impairment scores from infancy suggests that infant feeding methods and SDB are linked (infant sleep impairment survey was composed of 10 SDB-related questions). Although this is not a suitable replacement for PSG-validated methodology, coupled with the chi-square PSG snoring and "primary snoring" diagnosis finding provides moderate evidence linking infant feeding methods with SDB.

The proposed link between SDB and REM sleep was not directly established. Without correction for covariates, there was a statistical trend $(p=0.070)$ suggesting that greater infant sleep impairment scores were correlated with more REM sleep. This preliminary finding would be consistent with the negative correlation between breastfeeding duration and REM sleep, in that a reduction of REM sleep is more indicative of more mature, developed sleep. However, the trend was lost when covariates were corrected for using partial correlation. Also, despite the correct direction, a Mann-Whitney $U$ test confirmed that there was no significant difference in REM sleep (\%TST) between those with "primary snoring" and those without $(p=0.151)$.

Although breastfeeding duration was negatively correlated with REM sleep, as expected there was no significant correlation between breastfeeding duration and quiet sleep $(p=$ 0.531). We thus sought to compare breastfeeding duration and how it may or may not be correlated with active sleep ratio (active sleep/quiet sleep). It was ultimately concluded that after correcting for covariates, there was no significant correlation between the two. This may be interpreted to mean that, although there is sufficient study power to show there is greater REM sleep in infants with more breastfeeding duration, there is not yet enough power to conclude this if REM is taken as a ratio of quiet sleep specifically. 
In order to summarize the results from hypothesis $3 b$, I refer the reader to Fig 9. In this dissertation, the results suggest there is a correlation between breastfeeding duration and SDB severity, particularly SDB during REM sleep (those with "primary snoring" had significantly higher snore arousal index during active sleep than those without). A negative correlation between breastfeeding duration and SDB severity has been reported previously (MontgomeryDowns et al., 2007). In this dissertation, breastfeeding duration was also negatively correlated with REM sleep (\%TST), the opposite of which was hypothesized. Although I had originally predicted that a higher breastfeeding duration would be correlated with reduced SDB (supported) and that reduced SDB would be correlated with reduced REM sleep (\%TST), it may be that SDB simply fragments REM sleep, and that this fragmentation reduces the quality therefore necessitating a greater quantity of REM sleep to compensate. Indeed, at least in adult populations, selective REM deprivation leads to a REM-specific rebound effect (Beersma et al., 1990). Although fragmentation is not deprivation, disruption of the biological function of REM sleep could nonetheless accomplish a similar effect.

Not supported in this dissertation was the hypothesized direct association between SDB and REM sleep (\%TST). It may be that in this particular study there were insufficient children with severe enough SDB to support this effect. Demonstration of this link is critical to providing the necessary support to further the idea that SDB might mediate the correlation between breastfeeding duration and REM sleep (\%TST). 
Fig 10 contains a summary of all hypothesized findings in this dissertation. Using the model originally proposed in this dissertation, there were no significant findings in Specific Aim 1 (Fig 2). CC volumes were not correlated with breastfeeding duration, SDB severity, or neurocognitive outcomes. Although there is some evidence that re-investigation with greatly elevated sample sizes may be worthwhile (post-hoc power analysis), for the moment we must conclude that CC volume is not related to these variables. Likewise, breastfeeding and SDB were not found to be statistically correlated with neurocognitive measures, although some of this is likely the result of the confounding effect of more children diagnosed with ADHD (Perfect et al., 2013; Semrud-Clikeman, Walkowiak, Wilkinson, \& Butcher, 2010) in the sample than anticipated. Although some ADHD children in this sample were expected due to the association between SDB and ADHD-like behavior (Chervin et al., 2002; Jan et al., 2011; Perfect et al., 2013), a proportion of over $50 \%$ children with ADHD diagnosis was not anticipated. Based on previous literature, a proportion of no more than $35-40 \%$ was anticipated (Perfect et al., 2013).

The finding that CC volume was not correlated with breastfeeding duration was replicated in Specific Aim 2. However, an investigation in DTI revealed that breastfeeding duration was in fact positively correlated with FA scores from two major white matter pathways; LSLF (temporal portion) and LAB. Consistent with the literature, these pathways seem to be related to language and reading function, as well as spatial and verbal memory, which may help to explain neurocognitive changes in other studies. Academic performance was not found to be correlated with either breastfeeding duration or FA scores in this study, but there was a significant correlation found between breastfeeding duration and work habits/selfmanagement scores, providing weak evidence that breastfeeding duration may eventually 
affect behavior. There is a plethora of research to support that breastfeeding is correlated with elevated neurocognitive performance in children (For example, Anderson, Johnstone, \& Remley, 1999; Horwood \& Fergusson, 1998; Isaacs et al., 2010; Kramer, 2008; McCrory \& Murray, 2013; Oddy, Li, Whitehouse, Zubrick, \& Malacova, 2011). Therefore, although there is weak support for a correlation between breastfeeding and behavior in this study, the strongest finding was the correlation between breastfeeding duration and white matter FA scores in the left SLF and left cingulum angular bundle. Fractional anisotropy scores, particularly those in the left longitudinal fasciculus, have been correlated with elevated neurocognitive performance previously (Kamali et al., 2014; Karlsgodt et al., 2008; Nagae et al., 2012; Vestergaard et al., 2011)

In the third aim, there were few significant findings with regard to hypothesis $3 a$. This was largely due to a combination of small sample size, and a general lack of SDB in the sample.

In the second portion of the third aim, breastfeeding duration was negatively correlated with REM sleep (\%TST), which was the opposite direction than predicted. Additionally, it was found that exclusively formula-fed infants are more likely to snore on PSG and be diagnosed with "primary snoring" than those with any breastfeeding. Those with "primary snoring" had a significantly greater snore arousal index during REM sleep specifically, suggestive that REM sleep is specifically disrupted in individuals with SDB, at least in this young sample.

The main results of this dissertation is described in Figure 17. Red empty arrows are links which I hypothesize to exist, but have not yet been supported. Several of these links were simply not addressed in this dissertation (link between REM sleep and white matter), while others such as SDB severity and REM sleep (\%TST) and REM fragmentation and REM sleep 
(\%TST) simply were not statistically significant. Based on the results, it could be argued that a lack of power contributed to this as those with "primary snoring" had non-significantly greater REM sleep (\%TST) than those without $(p=0.151)$. In addition, when covariates were not controlled, there was a statistical trend that infant sleep impairment scores were positively correlated with REM sleep (\%TST; $p=0.070 ; r=0.385$ ).

Ultimately, how SDB, REM fragmentation, and REM sleep (\%TST) is potentially associated with REM sleep quality will have to be examined in the future to determine whether this hypothesis is supported. Improved REM sleep quality, perhaps rather than REM sleep quantity, may be linked to white matter in the brain as predicted in the ontogenetic hypothesis (Roffwarg et al., 1966). Obviously, a direct link between REM sleep and white matter volume in the CC or FA scores in the LSLF and LAB have yet to be established.

Not yet discussed fully is the lack of any significant correlations with CC volumes in either aim. Despite the fact that significant correlations were found using DTI, it was really the CC volumes that were central to these original hypotheses. Nevertheless, even though differences in volume were theoretically in the correct direction, often they were not close to significantly different or correlated. There could really be two reasons for this; either no correlation exists between breastfeeding duration, SDB severity, and CC volume, or the current study had insufficient power to investigate this phenomenon. It may also be, as stated earlier, that the white matter effect that was predicted is more localized to individual left-lateralized pathways associated with specific cognitive functions (Kamali et al., 2014; Karlsgodt et al., 2008; Nagae et al., 2012; Vestergaard et al., 2011), rather than a more global white matter effect that I was expecting with the CC. 
In some sense, the lack of statistically significant findings regarding the $\mathrm{CC}$ is not surprising in aim 2. If the reader will recall, I had predicted a partial mediation effect in aim 1 whereby SDB partially mediated the positive correlation between breastfeeding duration and CC volume. Since this correlation between breastfeeding duration and CC volume was not found to be significant in aim 1, where SDB in the sample was predicted to partially mediate, then it is unsurprising that breastfeeding duration and CC volume could not be found to be significant in aim 2, where SDB was not in the sample to mediate the two. However, it should be noted that since nothing was found to be significant in either aim, and that the sample sizes were not large enough, definitive conclusions regarding CC volumes in this study are difficult to ascertain.

\subsection{Limitations}

There are several important limitations that should be mentioned. First, all of the results in this dissertation are correlational in nature; causation cannot be deduced. Second, the method for measurement of breastfeeding duration, maternal recall, is prone to error, particularly years after the fact. Nevertheless, the mode of infant feeding method is most likely correct, but the accuracy of the reported duration of those methods could be questioned. Looking at the confidence rating given by parents when reporting infant feeding methods, it was found in all three aims that parents overwhelmingly reported a " 4 " or a " 5 " on the 5-point scale, where 5 is the highest confidence. This suggests parents were fairly confident. A third general limitation is that, in regards to MR scan type, not all of the scans had exactly the same 
parameters, although they were the same general type of scan. Also, not all of the scans had the CC within the field of view, excluding otherwise usable scans.

A fourth general limitation is that the sample sizes in all 3 aims, but particularly the first two were really too low to detect an effect. Post-hoc power analyses are described above, but in summary finding an effect between breastfeeding duration and CC volumes using collected data as model would require several hundred participants (not really feasible), but finding an effect between SDB metrics and CC volumes may be found with a population of 50 or so (feasible).

More specific limitations include motion sensitivity of DTI in the second aim. Differences in DTI have been reported in the past simply due to group differences in head movement, rather than actual underlying white matter differences (Yendiki et al., 2013). However, the current study should be protected from this limitation, as significant FA findings were controlled for head translation and rotation. The issue of motion sensitivity is nonetheless worth mentioning, considering that I used an unsedated pediatric sample.

It could be argued that the negative correlation between breastfeeding duration and REM sleep (\%TST) is an artifact of close proximity of breastfeeding mothers to their children compared to non-breastfeeding mothers, as close proximity to the mother itself may result in more sleep disturbance (Hunsley \& Thoman, 2002). Although this interpretation cannot be absolutely disputed, there was not a significant correlation found between breastfeeding duration and quiet sleep $(p=0.531)$. Although it may be more likely that a sleeping infant will be aroused from external stimulation in REM sleep (Langford, Meddis, \& Pearson, 1974), it is unlikely that the difference between the two stages would be so striking, and that REM sleep 
specifically is impeded while there is no effect on quiet sleep. Furthermore, several studies have found that arousal threshold between REM and NREM sleep are fairly equal (Ermis, Krakow, \& Voss, 2010; Keefe, Johnson, \& Hunter, 1971).

The result in aim 3 where breastfeeding duration was shown to be negatively correlated with \% REM sleep (\%TST) could be challenged in that the actual measurements were taken at 89 months of age in the infants, yet breastfeeding duration was solicited out to 24 months of age through retrospective report. This leaves the caveat that, although infants breastfed at a longer duration, they would have only been breastfed up until the time of the PSG measurements. In order to help address this, I also used an analysis of covariance to divide the infants that would have been still breastfeeding at the time of the study (greater than 10 months) and infants either no longer breastfed, or in the process of weaning at the time of the study (10 months or less than 10 months). As reported in the results, re-analyzing the data in this way still revealed a significant difference between groups $(p=0.049)$

Interpreting the negative correlation between breastfeeding duration and REM sleep (\%TST) with this limitation in mind, it may be that mothers who went on to breastfeed for longer durations (12-24 months) were more likely to breastfeed exclusively, or if both breastfeeding and formula was used, more likely to use breastfeeding as the primary feeding method in the first 8-9 months compared to mothers who breastfed a lesser overall duration. Another interpretation may be that mothers who breastfed for longer duration (12-24 months) were more vigilant in breastfeeding and therefore gave more daily feedings to their child (for example, gave them more expressed breast milk) for the first 8-9 months than mothers who breastfed a shorter duration. Additionally, when mothers reported that their child was 
breastfed, the proportion of feedings from active breastfeeding vs. expressed milk from a bottle is unknown. Regardless, the statistically significant negative correlation $(p=0.006)$ between breastfeeding duration and REM sleep (\%TST) in the first 8-9 months can likely be partially explained by an actual increase in either breast milk exposure or breastfeeding action by the infant during that time period.

Finally, the PSG study initially proposed for Specific Aim 3 was conducted, but did not go as planned. Although participants were screened so that only SDB-suspected individuals would participate, no significant PSG-validated SDB was detected, necessitating the alternative study for aim 3. Despite this, the initial component of aim 3 provided valuable personal experience as it gave me the opportunity to learn PSG application on pediatric and infant populations, and also learn to score PSG studies.

The second portion of aim 3 was constructed to answer the same research question as the original plan for aim 3. Here, a PSG study on 8-9 month old infants completed by Dr. Montgomery-Downs was selected to serve this purpose. I had constructed surveys to send out to participants to acquire breastfeeding information, demographics, and SDB suggestive questions based on the previous survey design used in this study. After IRB approval, these surveys were mailed, returned, processed, and analyzed. In the process, I learned a plethora of new statistical analysis techniques. Therefore, although the initial PSG data was not collected by me in this portion, I had completed all other aspects of the study.

\subsection{Future Directions}


In conclusion, although only a few main hypotheses were adequately supported, this dissertation provides an initial basic framework for further studies related to infant feeding methods, SDB, REM sleep, and white matter volume/FA scores. Eventually, given the substantial neurocognitive and developmental problems associated with SDB, I would like to see a theoretical framework spanning from infant-factors through sleep, brain development, and eventually cognition and behavior that can provide a model for how these deficits may be occurring. Ultimately, this leads to the developmental question of how sleep can ultimately affect behavior through an anatomical and physiological mechanism. Such a question, though complex, can begin to be answered or at least narrowed through careful scientific study. Only after the full scale of the issue is adequately realized can at-risk individuals be more properly identified and treated, and public awareness bolstered once the full implications of decisions during infancy and childhood (such as breastfeeding, and prevention/treatment of SDB) are revealed. With an enhanced understanding of the influence of SDB and sleep on the brain, and how to ensure the highest quality sleep possible for young children, I hope that the issues associated with SDB and poor sleep can one day be more properly managed. 
Allada, R., \& Siegel, J. M. (2008). Unearthing the Phylogenetic Roots of Sleep. Current Biology, 18(15), 14-20. http://doi.org/10.1016/j.cub.2008.06.033

Anders, T. F., Emde, R. N., \& Parmelee, A. H. (1971). A manual of standardized terminology, techniques and criteria for scoring of states of sleep and wakefulness in newborn infants. UCLA Brain Information Service Publications Office.

Anderson, A., \& Burggren, A. (2014). Cognitive and neurodevelopmental benefits of extended formulafeeding in infants: Re: Deoni et al. 2013. Neurolmage, 100, 706-709. http://doi.org/10.1016/j.neuroimage.2014.05.011

Anderson, J. W., Johnstone, B. M., \& Remley, D. T. (1999). Breast-feeding and cognitive development : a meta-analysis. The American Journal of Clinical Nutrition, 70, 525-535.

Archbold, K. H. (2013). Pediatric Sleep Apnea and Adherence to Positive Airway Pressure (PAP) Therapy. In A. R. Wolfson \& H. E. Montgomery-Downs (Eds.), The Oxford Handbook of Infant, Child, and Adolescent Sleep and Behavior (p. 362). New York, USA: Oxford University Press.

Archbold, K. H., Giordani, B., Ruzicka, D. L., \& Chervin, R. D. (2004). Cognitive executive dysfunction in children with mild sleep-disordered breathing. Biological Research for Nursing, 5(3), 168-176.

Archbold, K. H., \& Parthasarathy, S. (2009). Adherence to positive airway pressure therapy in adults and children. Current Opinion in Pulmonary Medicine, 15(6), 585-590.

Arzt, M., Young, T., Finn, L., Skatrud, J. B., \& Bradley, T. D. (2005). Association of sleep-disordered breathing and the occurrence of stroke. American Journal of Respiratory and Critical Care Medicine, 172(11), 1447-1451. http://doi.org/10.1164/rccm.200505-7020C

Aserinsky, E., \& Kleitman, N. (1953a). Eye movements during sleep. Federation Proceedings, 12, 6-7.

Aserinsky, E., \& Kleitman, N. (1953b). Regularly Occurring Periods of Eye Motility, and Concomitant Phenomena, during sleep. Science (New York, N.Y.), 118(3062), 4-5. http://doi.org/10.1126/science.118.3062.273

Aserinsky, E., \& Kleitman, N. (1955a). A Motility Cycle in Sleeping Infants as Manifested by Ocular and Gross Bodily Activity. Journal of Applied Physiology, 8(1), 11-18.

Aserinsky, E., \& Kleitman, N. (1955b). Two types of ocular motility occurring in sleep. Journal of Applied Physiology, 8(1), 1-10.

Ashtari, M., Kumra, S., Bhaskar, S. L., Clarke, T., Thaden, E., Cervellione, K. L., ... Ardekani, B. A. (2005). Attention-deficit/hyperactivity disorder: A preliminary diffusion tensor imaging study. Biological Psychiatry, 57(5), 448-455. http://doi.org/10.1016/j.biopsych.2004.11.047

Aurora, R. N., Chowdhuri, S., Ramar, K., Bista, S. R., Casey, K. R., Lamm, C. I., ... Tracy, S. L. (2012). The treatment of central sleep apnea syndromes in adults: practice parameters with an evidence-based literature review and meta-analyses. Sleep, 35(1), 17-40. http://doi.org/10.5665/sleep.1580

Ayalon, L., Liu, L., \& Ancoli-Israel, S. (2004). Diagnosing and treating sleep disorders in the older adult.pdf. Medical Clinics of North America, 88(3), 1-11.

Barnes, M. E., Huss, E. A., Garrod, K. N., Raay, E. Van, Dayyat, E., \& Gozal, D. (2009). Impairments in 
Attendtion in Occasionally Snoring Children: An Event-Related Potential Study. Dev Neuropsychol., 34(5), 629-649. http://doi.org/10.1016/j.str.2010.08.012.Structure

Becker, P. M., \& Forester, M. D. (2007). Periodic Leg Movements of Sleep. In N. Butkov \& T. Lee-Chiong (Eds.), Fundamentals of Sleep Technology (pp. 141-153). Philadelphia, PA: Lippincott Willaims \& Wilkins.

Beebe, D. W., \& Gozal, D. (2002). Obstructive sleep apnea and the prefrontal cortex: towards a comprehensive model linking nocturnal upper airway obstruction to daytime cognitive and behavioral deficits. Journal of Sleep Research, 11(1), 1-16. http://doi.org/doi:10.1046/j.13652869.2002.00289.x

Beersma, D. G. M., Dijk, D. J., Blok, C. G. H., \& Everhardus, I. (1990). REMS deprivation during 5 hours leads to an immediate REMS rebound and to suppression of non-REMS intensity. Electroencephalography and Clinical Neurophysiology, 76, 114-122. http://doi.org/10.1016/00134694(90)90209-3

Berrien, F. K. (1930). Recall of dreams during the sleep period. The Journal of Abnormal and Social Psychology, 25(2), 110-114.

Berry, R. B., Brooks, R., Gamaldo, C. E., Harding, S. M., Lloyd, R. M., Marcus, C. L., \& Vaughn, B. V. (2015). The AASM Manual for the Scoring of Sleep and Associated Events: Rules, Terminology, and Technical Specifications (Version 2.). Darien, IL 60561: American Academy of Sleep Medicine.

Berry, R. B., Gamaldo, C. E., Harding, S. M., Lloyd, R. M., Marcus, C. L., \& Vaughn, B. V. (2015). The AASM Manual for the Scoring of Sleep and Associated Events (Version 2.). Darien, IL 60561: American Academy of Sleep Medicine.

Biggs, S. N., Walter, L. M., Nisbet, L. C., Jackman, A. R., Anderson, V., Nixon, G. M., ... Horne, R. S. C. (2012). Time course of EEG slow-wave activity in pre-school children with sleep disordered breathing: A possible mechanism for daytime deficits? Sleep Medicine, 13(8), 999-1005. http://doi.org/10.1016/j.sleep.2012.05.006

Blink, E. J. (2010). MRI : Physics.

Blunden, S. L. (2012). Behavioural Sleep Disorders across the Developmental Age Span: An Overview of Causes, Consequences and Treatment Modalities. Psychology, 3(3), 249-256. http://doi.org/10.4236/psych.2012.33035

Bonuck, K. a, Chervin, R. D., Cole, T. J., Emond, A., Henderson, J., Xu, L., \& Freeman, K. (2011). Prevalence and persistence of sleep disordered breathing symptoms in young children: a 6-year populationbased cohort study. Sleep, 34(7), 875-884. http://doi.org/10.5665/sleep.1118

Bonuck, K., \& Grant, R. (2012). Sleep problems and early developmental delay: Implications for early intervention programs. Intellectual and Developmental Disabilities, 50(1), 41-52. http://doi.org/10.1352/1934-9556-50.1.41

Bourke, R. S., Anderson, V., Yang, J. S. C., Jackman, A. R., Killedar, A., Nixon, G. M., ... Horne, R. S. C. (2011a). Cognitive and academic functions are impaired in children with all severities of sleepdisordered breathing. Sleep Medicine, 12(5), 489-496. http://doi.org/10.1016/j.sleep.2010.11.010 
Bourke, R. S., Anderson, V., Yang, J. S. C., Jackman, A. R., Killedar, A., Nixon, G. M., ... Horne, R. S. C. (2011b). Neurobehavioral function is impaired in children with all severities of sleep disordered breathing. Sleep Medicine, 12(3), 222-229. http://doi.org/10.1016/j.sleep.2010.08.011

Brew, B. K., Marks, G. B., Almqvist, C., Cistulli, P. A., Webb, K., \& Marshall, N. S. (2014). Breastfeeding and snoring: A birth cohort study. PLOS ONE, 9(1). http://doi.org/10.1371/journal.pone.0084956

Buttoo, K., Pandi-Perumal, S. R., \& Guilleminault, C. (2016). Narcolepsy and Mental Illness. In M. Goswami, M. J. Thorpy, \& S. R. Pandi-Perumal (Eds.), Narcolepsy: A Clinical Guide (2nd ed., pp. 265-275). Springer International Publishing. http://doi.org/10.1007/978-3-319-23739-8

Byars, K. C., Yolton, K., Rausch, J., Lanphear, B., \& Beebe, D. W. (2011). Prevalence, Patterns, and Persistence of Sleep Problems in the First 3 Years of Life. Pediatrics, 129(2), e276-e284. http://doi.org/10.1542/peds.2011-0372

Carskadon, M. A., \& Dement, W. C. (2011). Normal Human Sleep : An overview. In M. H. Kryger, T. Roth, \& W. C. Dement (Eds.), Principles and practice of sleep medicine (5th ed., pp. 16-26). St. Louis: Elsevier Saunders.

Castronovo, V. E., \& Butkov, N. (2007). Sleep Stages and Sleep Architecture. In N. Butkov \& T. Lee-Chiong (Eds.), Fundamentals of Sleep Technology (pp. 280-299). Philadelphia, PA: Lippincott Willaims \& Wilkins.

Chen, R., Clifford, A., Lang, L., \& Anstey, K. J. (2013). Is exposure to secondhand smoke associated with cognitive parameters ofchildren and adolescents?-a systematic literature review. Annals of Epidemiology, 23(10), 652-661. http://doi.org/10.1016/j.annepidem.2013.07.001

Chervin, R. D., Archbold, K. H., Dillon, J. E., Panahi, P., Pituch, K. J., Dahl, R. E., \& Guilleminault, C. (2002). Inattention, hyperactivity, and symptoms of sleep-disordered breathing. Pediatrics, 109(3), 449456. http://doi.org/10.1542/peds.109.3.449

Chervin, R. D., Ruzicka, D. L., Archbold, K. H., \& Dillon, J. E. (2005). Snoring predicts hyperactivity four years later. Sleep, 28(7), 885-890.

Chervin, R. D., Ruzicka, D. L., Giordani, B., Weatherly, R. A., Dillon, J. E., Hodges, E. K., ... Guire, K. E. (2006). Sleep-Disordered Breathing, Behavior, and Cognition in Children Before and After Adenotonsillectomy. Pediatrics, 117(4), e769-e778. http://doi.org/10.1542/peds.2005-1837

Chu, C. J., Leahy, J., Pathmanathan, J., Kramer, M. A., \& Cash, S. S. (2014). The maturation of cortical sleep rhythms and networks over early development. Clinical Neurophysiology, 125(7), 1360-1370. http://doi.org/10.1016/j.clinph.2013.11.028

Colen, C. G., \& Ramey, D. M. (2014). Is breast truly best? Estimating the effects of breastfeeding on longterm child health and wellbeing in the United States using sibling comparisons. Social Science and Medicine, 109, 55-65. http://doi.org/10.1016/j.socscimed.2014.01.027

Comella, C. L. (2014). Treatment of Restless Legs Syndrome. Neurotherapeutics, 11(1), 177-187. http://doi.org/10.1007/s13311-013-0247-9

Cox, R. W. (1996). AFNI: software for analysis and visualization of functional magnetic resonance neuroimages. Computers and Biomedical Research, 29(3), 162-173. 
Daurat, A., Foret, J., Bret-Dibat, J.-L., Fureix, C., \& Tiberge, M. (2008). Spatial and temporal memories are affected by sleep fragmentation in obstructive sleep apnea syndrome. Journal of Clinical and Experimental Neuropsychology, 30(1), 91-101. http://doi.org/10.1080/13803390701236116

Deoni, S. C. L., Dean, D. C., Piryatinsky, I., O’Muircheartaigh, J., Waskiewicz, N., Lehman, K., ... Dirks, H. (2013). Breastfeeding and early white matter development: A cross-sectional study. Neurolmage, 82, 77-86. http://doi.org/10.1016/j.neuroimage.2013.05.090

Deutsch, G. K., Dougherty, R. F., Bammer, R., Siok, W. T., Gabrieli, J. D. E., \& Wandell, B. (2005). Children's reading performance is correlated with white matter structure measured by diffusion tensor imaging. Cortex, 41, 354-363. http://doi.org/10.1016/S0010-9452(08)70272-7

Dunn, L. M., \& Dunn, D. M. (2007). PPVT-4: Peabody picture vocabulary test (4th Editio). Minneapolis, MN: Pearson.

Ellen, R. L., Marshall, S. C., Palayew, M., Molnar, F. J., Wilson, K. G., \& Man-Son-Hing, M. (2006). Systematic review of motor vehicle crash risk in persons with sleep apnea. J Clin Sleep Med, 2(2), 193-200. Retrieved from http://www.ncbi.nlm.nih.gov/entrez/query.fcgi?cmd=Retrieve\&db=PubMed\&dopt=Citation\&list_u ids $=17557495$

Engler, A. C., Hadash, A., Shehadeh, N., \& Pillar, G. (2012). Breastfeeding may improve nocturnal sleep and reduce infantile colic: Potential role of breast milk melatonin. European Journal of Pediatrics, 171(4), 729-732. http://doi.org/10.1007/s00431-011-1659-3

Ermis, U., Krakow, K., \& Voss, U. (2010). Arousal thresholds during human tonic and phasic REM sleep. Journal of Sleep Research, 19(3), 400-406. http://doi.org/10.1111/j.1365-2869.2010.00831.x

Ezzati, A., Katz, M. J., Lipton, M. L., Zimmerman, M. E., \& Lipton, R. B. (2016). Hippocampal volume and cingulum bundle fractional anisotropy are independently associated with verbal memory in older adults. Brain Imaging and Behavior, 10(3), 652-659. http://doi.org/10.1007/s11682-015-9452-y

Faul, F., Erdfelder, E., Lang, A. G., \& Buchner, A. (2007). A flexible statistical power analysis program for the social, behavioral, and biomedical sciences. Behavior Research Methods, 39, 175-191.

Fischl, B., Van Der Kouwe, A., Destrieux, C., Halgren, E., Ségonne, F., Salat, D. H., ... Dale, A. M. (2004). Automatically Parcellating the Human Cerebral Cortex. Cerebral Cortex, 14(1), 11-22. http://doi.org/10.1093/cercor/bhg087

Frazier, T. W., Keshavan, M. S., Minshew, N. J., \& Hardan, A. Y. (2012). A two-year longitudinal MRI study of the corpus callosum in autism. Journal of Autism and Developmental Disorders, 42(11), 23122322. http://doi.org/10.1007/s10803-012-1478-z

Friedman, B.-C., Hendeles-Amitai, A., Kozminsky, E., Leiberman, A., Friger, M., Tarasiuk, A., \& Tal, A. (2003). Adenotonsillectomy improves neurocognitive function in children with obstructive sleep apnea syndrome. Sleep, 26(8), 999-1005.

Galbally, M., Lewis, A. J., McEgan, K., Scalzo, K., \& Islam, F. A. (2013). Breastfeeding and infant sleep patterns: An Australian population study. Journal of Paediatrics and Child Health, 49(2), 147-152. http://doi.org/10.1111/jpc.12089 
Gerstadt, C. L., Hong, Y. J., \& Diamond, A. (1994). The relationship between cognition and action: performance of children 3 1/2 - 7 years old on a Stroop-like day-night test. Congition, 53, 129-153.

Giacomini, A., Tognoli, R. L., \& Hydes, N. (2007). Narcolepsy. In N. Butkov \& T. Lee-Chiong (Eds.), Fundamentals of Sleep Technology (pp. 159-167). Philadelphia, PA: Lippincott Willaims \& Wilkins.

Gibbs, B. G., \& Forste, R. (2014). Socioeconomic status, infant feeding practices and early childhood obesity. Pediatric Obesity, 9(2), 135-146. http://doi.org/10.1111/j.2047-6310.2013.00155.x

Giedd, J. N., Blumenthal, J., Jeffries, N., Rajapakse, J. C., Vaituzis, A. C., Liu, H., ... Castellanos, F. X. (1999). Development of the human corpus callosum during childhood and adolescence: a longitudinal mri study. Proceedings in Neuro-Psychopharmacology \& Biological Psychiatry, 23, 571-588.

Giedd, J. N., \& Rapoport, J. L. (2010). Structural MRI of Pediatric Brain Development: What Have We Learned and Where Are We Going? Neuron, 67(5), 728-734. http://doi.org/10.1016/j.neuron.2010.08.040

Giedd, J. N., Rumsey, J. M., Castellanos, F. X., Rajapakse, J. C., Kaysen, D., Catherine Vaituzis, A., ... Rapoport, J. L. (1996). A quantitative MRI study of the corpus callosum in children and adolescents. Developmental Brain Research, 91(2), 274-280. http://doi.org/10.1016/0165-3806(95)00193-X

Gioia, G. A., Isquith, P. K., Guy, S. C., \& Kenworthy, L. (2000). Behavioral rating of executive function: BRIEF. Odessa, FL: Psychological Assessment Resources.

Gislason, T., \& Benediktsdóttir, B. (1995). Snoring, Apneic Episodes, and Nocturnal Hypoxemia Among Children 6 Months to 6 Years Old. Chest, 107(4), 963-966. http://doi.org/10.1378/chest.107.4.963

Goldbart, A., Peppard, P., Finn, L., Ruoff, C. M., Barnet, J., Young, T., \& Mignot, E. (2014). Narcolepsy and predictors of positive MSLTs in the Wisconsin Sleep Cohort. Sleep, 37(6), 1043-51. http://doi.org/10.5665/sleep.3758

Gozal, D., \& Pope Jr., D. W. (2001). Snoring During Early Childhood and Academic Performance at Ages Thirteen to Fourteen Years. Pediatrics, 107(6), 1394-1399. http://doi.org/10.1542/peds.107.6.1394

Gozal, D., Wang, M., \& Pope Jr., D. W. (2001). Objective sleepiness measures in pediatric obstructive sleep apnea. Pediatrics, 108(3), 693-697. http://doi.org/10.1542/peds.108.3.693

Grigg-Damberger, M., \& Ralls, F. (2012). Cognitive dysfunction and obstructive sleep apnea: from cradle to tomb. Current Opinion in Pulmonary Medicine, 18(6), 580-7. http://doi.org/10.1097/MCP.0b013e328358be18

Guilleminault, C., Benbir, G., \& Akhtar, N. (2007). Obstructive Sleep Apnea. In N. Butkov \& T. Lee-Chiong (Eds.), Fundamentals of Sleep Technology (pp. 113-123). Philadelphia, PA: Lippincott Willaims \& Wilkins.

Guilleminault, C., Lee, J., \& Chan, A. (2005). Pediatric obstructive sleep apnea syndrome. Arch Pediatr Adolesc Med, 159(8), 775-85.

Haacke, E. M., Brown, R. W., Thompson, M. R., \& Venkatesan, R. (1999). Magnetic Resonance Imaging. (I. John Wiley \& Sons, Ed.). New York, NY. 
Haba-Rubio, J., Marti-Soler, H., Marques-Vidal, P., Tobback, N., Andries, D., Preisig, M., ... Heinzer, R. (2016). Prevalence and determinants of periodic limb movements in the general population. Annals of Neurology, 79(3), 464-474. http://doi.org/10.1002/ana.24593

Halbower, A. C., Degaonkar, M., Barker, P. B., Earley, C. J., Marcus, C. L., Smith, P. L., ... Mahone, E. M. (2006). Childhood obstructive sleep apnea associates with neuropsychological deficits and neuronal brain injury. PLoS Medicine, 3(8), 1391-1402. http://doi.org/10.1371/journal.pmed.0030301

Hamilton, L. S., Levitt, J. G., Neill, J. O., Alger, J. R., Luders, E., Phillips, O. R., ... Katherine, L. (2008). Reduced white matter integrity in attention-deficit hyperactivity disorder. Neuroreport, 19(17), 19. http://doi.org/10.1097/WNR.0b013e3283174415.Reduced

Hardan, A. Y., Pabalan, M., Gupta, N., Bansal, R., Melhem, N. M., Fedorov, S., ... Minshew, N. J. (2009). Corpus callosum volume in children with autism. Psychiatry Research: Neuroimaging, 174(1), 5761. http://doi.org/10.1016/j.pscychresns.2009.03.005

Haut, M. W., Kuwabara, H., Ducatman, A. M., Hatfield, G., Parsons, M. W., Scott, A., ... Morrow, L. A. (2006). Corpus callosum volume in railroad workers with chronic exposure to solvents. Journal of Occupational and Environmental Medicine, 48(6), 615-624. http://doi.org/10.1097/01.jom.0000205211.67120.23

Horwood, L. J., \& Fergusson, D. M. (1998). Breastfeeding and later cognitive and academic outcomes. Pediatrics, 101(1), E9. http://doi.org/10.1542/peds.101.1.e9

Hunsley, M., \& Thoman, E. B. (2002). The sleep of co-sleeping infants when they are not co-sleeping: Evidence that co-sleeping is stressful. Developmental Psychobiology, 40(1), 14-22. http://doi.org/10.1002/dev.10009

Hunter, S. J., Gozal, D., Smith, D. L., Philby, M. F., Kaylegian, J., \& Kheirandish-Gozal, L. (2016). Effect of Sleep-Disordered Breathing Severity on Cognitive Performance Measures in a Large Community of Young School-aged Children. American Jounral of Respiratory and Critical Care Medicine, 194(6), 739-747. http://doi.org/10.1164/rccm.201510-20990C

Iglewicz, B., \& Banerjee, S. (2001). A simple univariate outlier identification procedure. Proceedings of the Annual Meeting of the American Statistical Association, 5-9. Retrieved from http://www.amstat.org/sections/SRMS/Proceedings/y2001/Proceed/00523.pdf

Isaacs, E. B., Fischl, B. R., Quinn, B. T., Chong, W. K., \& Gadian, D. G. (2010). Impact of breast milk on IQ, brain size and white matter development. Pediatric Research, 67(4), 357-362. http://doi.org/10.1203/PDR.0b013e3181d026da

Isaacs, E. B., Gadian, D. G., Sabatini, S., Chong, W. K., Quinn, B. T., Fischl, B. R., \& Lucas, A. (2008). The effect of early human diet on caudate volumes and IQ. Pediatric Research, 63(3), 308-314. http://doi.org/10.1203/PDR.0b013e318163a271

Jackman, A. R., Biggs, S. N., Walter, L. M., Embuldeniya, U. S., Davey, M. J., Nixon, G. M., ... Horne, R. S. C. (2012). Sleep-disordered breathing in preschool children is associated with behavioral, but not cognitive, impairments. Sleep Medicine, 13(6), 621-631.

http://doi.org/10.1016/j.sleep.2012.01.013 
Jacobsen, L. K., Picciotto, M. R., Heath, C. J., Frost, S. J., Tsou, K. A., Dwan, R. A., ... Mencl, W. E. (2007). Prenatal and adolescent exposure to tobacco smoke modulates the development of white matter microstructure. The Journal of Neuroscience, 27(49), 13491-13498. http://doi.org/10.1523/JNEUROSCI.2402-07.2007

Jan, Y.-W., Yang, C.-M., \& Huang, Y.-S. (2011). Comorbidity and confounding factors in attentiondeficit/hyperactivity disorder and sleep disorders in children. Psychology Research and Behavior Management, 4, 139-50. http://doi.org/10.2147/PRBM.S14055

Javaheri, S. (2010). Central sleep apnea. Clinics in Chest Medicine, 31(2), 235-248. http://doi.org/10.1016/j.ccm.2010.02.013

Johnson, M. H. (2001). Functional Brain Development in Humans. Nature Reviews Neuroscience, 2(7), 475-483. http://doi.org/10.1038/35081509

Joyner, K., Silver, C., \& Stavinoha, P. (2009). Relationship between parenting stress and ratings of executive functioning in children with ADHD. Journal of Psychoeducational Assessment, 27(6), 452464.

Kafouri, S., Kramer, M., Leonard, G., Perron, M., Pike, B., Richer, L., ... Paus, T. (2013). Breastfeeding and brain structure in adolescence. International Journal of Epidemiology, 42(1), 150-159. http://doi.org/10.1093/ije/dys172

Kamali, A., Flanders, A. E., Brody, J., Hunter, J. V, \& Hasan, K. M. (2014). Tracing superior longitudinal fasciculus connectivity in the human brain using high resolution diffusion tensor tractography. Brain Structure \& Function, 219(1), 269-81. http://doi.org/10.1007/s00429-012-0498-y

Karlsgodt, K. H., van Erp, T. G. M., Poldrack, R. A., Bearden, C. E., Nuechterlein, K. H., \& Cannon, T. D. (2008). Diffusion Tensor Imaging of the Superior Longitudinal Fasciculus and Working Memory in Recent-Onset Schizophrenia. Biological Psychiatry, 63(5), 512-518. http://doi.org/10.1016/j.biopsych.2007.06.017

Karni, A., Tanne, D., Rubenstein, B. S., Askenasy, J. J. M., \& Sagi, D. (1994). Dependence on REM Sleep of Overnight Improvement of a Perceptual Skill. Science, 265, 679-682.

Karpinski, A. C., Scullin, M. H., \& Montgomery-Downs, H. E. (2008). Risk for sleep-disordered breathing and executive function in preschoolers. Sleep Medicine, 9(4), 418-424. http://doi.org/10.1016/j.sleep.2007.06.004

Katz, L. C., \& Shatz, C. J. (1996). Synaptic activity and the construction of cortical circuits. Science, 274(5290), 1133-1138. http://doi.org/10.1126/science.274.5290.1133

Keefe, F. B., Johnson, L. C., \& Hunter, E. J. (1971). EEG and Autonomic response pattern during waking and sleep stages. Psychophysiology, 8(2), 198-212.

Keller, T. a, Kana, R. K., \& Just, M. A. (2007). A developmental study of the structural integrity of white matter in autism A developmental study of the structural integrity of white matter in autism. Neuroreport, 18(1), 23-27. http://doi.org/10.1097/01.wnr.0000239965.21685.99

Kent, J. C., Hepworth, A. R., Sherriff, J. L., Cox, D. B., Mitoulas, L. R., \& Hartmann, P. E. (2013). Longitudinal changes in breastfeeding patterns from 1 to 6 months of lactation. Breastfeeding 
Medicine, 8(4), 401-407. http://doi.org/10.1089/bfm.2012.0141

Keshavan, M. S., Diwadkar, V. a, DeBellis, M., Dick, E., Kotwal, R., Rosenberg, D. R., ... Pettegrew, J. W. (2002). Development of the corpus callosum in childhood, adolescence and early adulthood. Life Sciences, 70, 1909-1922. http://doi.org/10.1016/S0024-3205(02)01492-3

Key, A. P. F., Molfese, D. L., Brien, L. O., \& Gozal, D. (2009). NIH Public Access. Dev Neuropsychol., 34(5), 615-628. http://doi.org/10.1016/j.jneuroling.2010.09.006.Reduced

Kohler, M. J., Lushington, K., van den Heuvel, C. J., Martin, J., Pamula, Y., \& Kennedy, D. (2009). Adenotonsillectomy and neurocognitive deficits in children with sleep disordered breathing. PLOS ONE, 4(10). http://doi.org/10.1371/journal.pone.0007343

Kramer, M. S., Aboud, F. A., Mironova, E., Vanilovich, I., Platt, R. W., Matush, L., ... Shapiro, S. (2008). Breastfeeding and Child Cognitive Development. Archives of General Psychiatry, 65(5), 578. http://doi.org/10.1001/archpsyc.65.5.578

Langford, G. W., Meddis, R., \& Pearson, A. J. D. (1974). Awakening latency from sleep for meaningful and non-meaningful stimuli. Psychophysiology, 11(1), 1-5.

Leary, E. (2007). Patient preparation. In N. Butkov \& T. Lee-Chiong (Eds.), Fundamentals of Sleep Technology (pp. 241-252). Philadelphia, PA: Lippincott Willaims \& Wilkins.

Levy, K. J. (1980). A MONTE CARLO STUDY OF ANALYSIS OF COVARIANCE UNDER VIOLATIONS OF THE ASSUMPTIONS OF NORMALITY AND EQUAL REGRESSION SLOPES. Educational and Psychological Measurement, 40, 835-840.

Linley, L. A., Papadima, E., \& Fabiani, M. (2007). Central Sleep Apnea. In N. Butkov \& T. Lee-Chiong (Eds.), Fundamentals of Sleep Technology (pp. 124-130). Philadelphia, PA: Lippincott Willaims \& Wilkins.

Liston, C., Watts, R., Tottenham, N., Davidson, M. C., Niogi, S., Ulug, A. M., \& Casey, B. J. (2006). Frontostriatal microstructure modulates efficient recruitment of cognitive control. Cerebral Cortex, 16(4), 553-560. http://doi.org/10.1093/cercor/bhj003

Lumeng, J. C., \& Chervin, R. D. (2008). Epidemiology of pediatric obstructive sleep apnea. Proceedings of the American Thoracic Society, 5(2), 242-52. http://doi.org/10.1513/pats.200708-135MG

Mabbott, D. J., Noseworthy, M., Bouffet, E., Laughlin, S., \& Rockel, C. (2006). White matter growth as a mechanism of cognitive development in children. Neurolmage, 33(3), 936-946.

http://doi.org/10.1016/j.neuroimage.2006.07.024

Mahone, E. M., Cirino, P. T., Cutting, L. E., Cerrone, P. M., Hagelthorn, K. M., Hiemenz, J. R., ... Denckla, M. B. (2002). Validity of the behavior rating inventory of executive function in children with ADHD and / or Tourette syndrome. Archives of Clinical Neuropsychology, 17, 643-662.

Makris, N., Buka, S. L., Biederman, J., Papadimitriou, G. M., Hodge, S. M., Valera, E. M., ... Seidman, L. J. (2008). Attention and executive systems abnormalities in adults with childhood adhd: A DT-MRI study of connections. Cerebral Cortex, 18(5), 1210-1220. http://doi.org/10.1093/cercor/bhm156

Manconi, M., Ferri, R., Zucconi, M., Bassetti, C. L., Fulda, S., Aric??, D., \& Ferini-Strambi, L. (2012). 
Dissociation of periodic leg movements from arousals in restless legs syndrome. Annals of Neurology, 71(6), 834-844. http://doi.org/10.1002/ana.23565

Marcus, C. L., Brooks, L. J., Ward, S. D., Draper, K. A., Gozal, D., Halbower, A. C., ... Spruyt, K. (2012). Diagnosis and Management of Childhood Obstructive Sleep Apnea Syndrome. Pediatrics, 130(3), 714-755. http://doi.org/10.1542/peds.2012-1672

Marcus, C. L., Moore, R. H., Rosen, C. L., Giordani, B., Garetz, S. L., Taylor, H. G., ... Redline, S. (2013). A Randomized Trial of Adentonsillectomy for Childhood Sleep Apnea. N Engl J Med, 368(25), 23662376. http://doi.org/10.1056/NEJMoa1215881.A

Marcus, C. L., Traylor, J., Gallagher, P. R., Brooks, L. J., Huang, J., Koren, D., ... Tapia, I. E. (2014). Prevalence of periodic limb movements during sleep in normal children. Sleep, 37(8), 1349-1352. http://doi.org/10.5665/sleep.3928

Mares, D., McLuckie, A., Schwartz, M., \& Saini, M. (2007). Executive function impairments in children with attention-deficit hyperactivity disorder: do they differ between school and home environments? The Canadian Journal of Psychiatry, 52(8), 527-534. Retrieved from papers://fd97edd0-de1d-401e-87f3-202830b99eeb/Paper/p5524

Marks, G. A., Shaffery, J. P., Oksenberg, A., Speciale, S. G., \& Roffwarg, H. P. (1995). A functional role for REM sleep in brain maturation. Behavioural Brain Research, 69, 1-11. http://doi.org/10.1016/0166-4328(95)00018-0

Mazzotti, D. R., Guindalini, C., Moraes, W. A. dos S., Andersen, M. L., Cendoroglo, M. S., Ramos, L. R., \& Tufik, S. (2014). Human longevity is associated with regular sleep patterns, maintenance of slow wave sleep, and favorable lipid profile. Frontiers in Aging Neuroscience, 6(134), 1-9. http://doi.org/10.3389/fnagi.2014.00134

McCrory, C., \& Murray, A. (2013). The effect of breastfeeding on neuro-development in infancy. Maternal and Child Health Journal, 17(9), 1680-1688. http://doi.org/10.1007/s10995-012-1182-9

McFadden, A., \& Toole, G. (2006). Exploring women's views of breastfeeding: a focus group study within an area with high levels of socio-economic deprivation. Maternal and Child Nutrition, 2(3), 156168. Retrieved from http://ovidsp.ovid.com/ovidweb.cgi?T=JS\&PAGE=reference\&D=med5\&NEWS=N\&AN=16881928\n http://ovidsp.ovid.com/ovidweb.cgi?T=JS\&PAGE=reference\&D=emed7\&NEWS=N\&AN=200626741 0

Mignot, E., Lin, L., Rogers, W., Honda, Y., Qiu, X., Lin, X., ... Risch, N. (2001). Complex HLA-DR and -DQ interactions confer risk of narcolepsy-cataplexy in three ethnic groups. American Journal of Human Genetics, 68(3), 686-699. http://doi.org/10.1086/318799

Mindell, J. A., Du Mond, C., Tanenbaum, J. B., \& Gunn, E. (2012). Long-term relationship between breastfeeding and sleep. Children's Health Care, 41(3), 190-203. http://doi.org/10.1080/02739615.2012.685038

Ming, X., Sun, Y.-M., Nachajon, R. V, Brimacombe, M., \& Walters, A. S. (2009). Prevalence of parasomnia in autistic children with sleep disorders. Clinical Medicine. Pediatrics, 3, 1-10. Retrieved from http://www.pubmedcentral.nih.gov/articlerender.fcgi?artid=3676289\&tool=pmcentrez\&rendertyp $\mathrm{e}=\mathrm{abstract}$ 
Montgomery-Downs, H. E. (2013). Mechanism of Infant Feeding Method Protection against Pediatric Sleep-Disordered Breathing.

Montgomery-Downs, H. E., Crabtree, V. M., Capdevila, O. S., \& Gozal, D. (2007). Infant-Feeding Methods and Childhood Sleep-Disordered Breathing. Pediatrics, 120(5), 1030-1035. http://doi.org/10.1542/peds.2007-0722

Montgomery-Downs, H. E., Jones, V. F., Molfese, V. J., \& Gozal, D. (2003). Snoring in preschoolers: associations with sleepiness, ethnicity, and learning. Clinical Pediatrics, 42(8), 719-726. http://doi.org/10.1177/000992280304200808

Montgomery-Downs, H. E., O’Brien, L. M., Holbrook, C. R., \& Gozal, D. (2004). Snoring and sleepdisordered breathing in young children: subjective and objective correlates. Sleep, 27(1), 87-94. Retrieved from http://www.ncbi.nlm.nih.gov/pubmed/14998242

Morgenthaler, T. I., Owens, J., Alessi, C., Boehlecke, B., Brown, T. M., Coleman, J., ... Swick, T. J. (2006). Practice Parameters for Behavioral Treatment of Bedtime Problems and Night Wakings in Infants and Young Children. SLEEP, 29(10), 1277-1281.

Mortensen, E. L., Michaelsen, K. F., Sanders, S. a, \& Reinisch, J. M. (2002). The Association Between Duration of Breastfeeding and Adult Intelligence. JAMA: Journal of the American Medical Association, 287(18), 2365-2372.

Nagae, L. M., Zarnow, D. M., Blaskey, L., Dell, J., Khan, S. Y., Qasmieh, S., ... Roberts, T. P. L. (2012). Elevated mean diffusivity in the left hemisphere superior longitudinal fasciculus in autism spectrum disorders increases with more profound language impairment. American Journal of Neuroradiology, 33(9), 1720-1725. http://doi.org/10.3174/ajnr.A3037

Nagy, Z., Westerberg, H., \& Klingberg, T. (2004). Maturation of white matter is associated with the development of cognitive functions during childhood. Journal of Cognitive Neuroscience, 16(Figure 1), 1227-1233. http://doi.org/10.1162/0898929041920441

Nevsimalova, S., Prihodova, I., Kemlink, D., \& Skibova, J. (2013). Childhood parasomnia - A disorder of sleep maturation? European Journal of Paediatric Neurology, 17(6), 615-619. http://doi.org/10.1016/j.ejpn.2013.05.004

Nieto, F. J., Young, T. B., Lind, B. K., Redline, S., Agostino, R. B. D., Newman, A. B., ... Pickering, T. G. (2000). in a Large Community-Based Study for the Sleep Heart Health Study. JAMA, 284(14), 18291836.

Noble, K. G., Houston, S. M., Brito, N. H., Bartsch, H., Kan, E., Kuperman, J. M., ... Sowell, E. R. (2015). Family income, parental education and brain structure in children and adolescents. Nature Neuroscience, 18(5), 773-778. http://doi.org/10.1038/nn.3983

Northam, G. B., Liégeois, F., Chong, W. K., Wyatt, J. S., \& Baldeweg, T. (2011). Total brain white matter is a major determinant of IQ in adolescents born preterm. Annals of Neurology, 69(4), 702-711. http://doi.org/10.1002/ana.22263

Oddy, W. H., Li, J., Whitehouse, A. J. O., Zubrick, S. R., \& Malacova, E. (2011). Breastfeeding duration and academic achievement at 10 years. Pediatrics, 127(1), e137-e145.

http://doi.org/10.1542/peds.2009-3489 
Ohayon, M. M., Carskadon, M. A., Guilleminault, C., \& Vitiello, M. V. (2004). Meta-Analysis of Quantitative Sleep Parameters From Childhood to Old Age in Healthy Individuals: Developing Normative Sleep Values Across the Human Lifespan. Sleep, 27(7), 1255-73. Retrieved from http://www.ncbi.nlm.nih.gov/pubmed/15586779

Owens, J. A. (2009). Neurocognitive and behavioral impact of sleep disordered breathing in children. Pediatric Pulmonology, 44(5), 414-422. http://doi.org/10.1002/ppul.20981

Owens, J. A., \& Mohan, M. (2016). Behavioral Interventions for Parasomnias. Current Sleep Medicine Reports, 2(2), 81-86. http://doi.org/10.1007/s40675-016-0046-z

Paus, T., Nawazkhan, I., Leonard, G., Perron, M., Pike, G. B., Pitiot, A., ... Pausova, Z. (2008). Corpus callosum in adolescent offspring exposed prenatally to maternal cigarette smoking. Neurolmage, 40(2), 435-441. http://doi.org/10.1016/j.neuroimage.2007.10.066

Peppard, P. E., Young, T., Barnet, J. H., Palta, M., Hagen, E. W., \& Hla, K. M. (2013). Increased prevalence of sleep-disordered breathing in adults. American Journal of Epidemiology, 177(9), 1006-1014. http://doi.org/10.1093/aje/kws342

Perfect, M. M., Archbold, K., Goodwin, J. L., Levine-Donnerstein, D., \& Quan, S. F. (2013). Risk of behavioral and adaptive functioning difficulties in youth with previous and current sleep disordered breathing. Sleep, 36(4), 517-525B. http://doi.org/10.5665/sleep.2536

Pigeon, W. R. (2010). Treatment of Adult Insomnia with Cognitive-Behavioral Therapy. Journal of Clinical Psychology, 66(11), 1148-1160.

Piteo, A. M., Lushington, K., Roberts, R. M., Martin, A. J., Nettelbeck, T., Kohler, M. J., \& Kennedy, J. D. (2011). Parental-reported snoring from the first month of life and cognitive development at 12months of age. Sleep Medicine, 12(10), 975-980. http://doi.org/10.1016/j.sleep.2011.07.006

Preacher, K. J., \& Hayes, A. F. (2008). Asymptotic and resampling strategies for assessing and comparing indirect effects in multiple mediator models. Behavior Research Methods, 40(3), 879-891. http://doi.org/10.3758/BRM.40.3.879

Prigge, M. B. D., Lange, N., Bigler, E. D., Merkley, T. L., Neeley, E. S., Abildskov, T. J., ... Lainhart, J. E. (2013). Corpus callosum area in children and adults with autism. Research in Autism Spectrum Disorders, 7(2), 221-234. http://doi.org/10.1016/j.rasd.2012.09.007

Punjabi, N. M. (2016). COUNTERPOINT: Is the Apnea-Hypopnea Index the Best Way to Quantify the Severity of Sleep-Disordered Breathing? No. Chest, 149(1), 16-19. http://doi.org/10.1378/chest.14-2261

Punjabi, N. M., Bandeen-Roche, K., Marx, J. J., Neubauer, D. N., Smith, P. L., \& Schwartz, A. R. (2002). The association between daytime sleepiness and sleep-disordered breathing in NREM and REM sleep. Sleep, 25(3), 307-314.

Punjabi, N. M., O’Hearn, D., Neubauer, D., Nieto, F., Schwartz, A., Smith, P., \& Bandeen-Roche, K. (1999). Modeling Hypersomnolence in Sleep-disordered Breathing-A Novel Approach Using Survival Analysis. American Journal of Respiratory and Critical Care Medicine, 159, 1703-1709. Retrieved from http://ajrccm.atsjournals.org/content/159/6/1703.short 
Ramamurthy, M. B., Sekartini, R., Ruangdaraganon, N., Huynh, D. H. T., Sadeh, A., \& Mindell, J. A. (2012). Effect of current breastfeeding on sleep patterns in infants from Asia-Pacific region. Journal of Paediatrics and Child Health, 48(8), 669-674. http://doi.org/10.1111/j.1440-1754.2012.02453.x

Rechtschaffen, A., Gilliland, M. A., Bergmann, B. M., \& Winter, J. B. (1983). Physiological correlates of prolonged sleep deprivation in rats. Science, 221(4606), 182-184.

Redington, J. L., Mattice, C., \& Eckhardt, W. (2007). Parasomnias. In N. Butkov \& T. Lee-Chiong (Eds.), Fundamentals of Sleep Technology (pp. 168-173). Philadelphia, PA: Lippincott Willaims \& Wilkins.

Redline, S., Kirchner, H. L., Quan, S. F., Gottlieb, D. J., Kapur, V., \& Newman, A. (2004). The effects of age, sex, ethnicity, and sleep-disordered breathing on sleep architecture. Archives of Internal Medicine, 164(4), 406-18. http://doi.org/10.1001/archinte.164.4.406

Reitan, R. M., \& Wolfson, D. (1985). The Halstead-Reitan neuropsychological test battery: Therapy and clinical interpretation. Tucson, AZ: Neuropsychological Press.

Robison, J. G., Wilson, C., Otteson, T. D., Chakravorty, S. S., \& Mehta, D. K. (2013). Analysis of outcomes in treatment of obstructive sleep apnea in infants. Laryngoscope, 123(9), 2306-2314. http://doi.org/10.1002/lary.23685

Roffwarg, H. P., Muzio, J. N., \& Dement, W. C. (1966). Ontogenetic development of the human sleepdream cycle. Science, 152, 604-619. http://doi.org/10.1126/science.152.3722.604

Roth, T., \& Roehrs, T. (2003). Insomnia : Epidemiology , Characteristics, and Consequences. Clinical Cornerstone, 5(3), 5-12.

Sarris, J., \& Byrne, G. J. (2011). A systematic review of insomnia and complementary medicine. Sleep Medicine Reviews, 15(2), 99-106. http://doi.org/10.1016/j.smrv.2010.04.001

Schade, M. M., Bauer, C. E., Warren, C. N., \& Montgomery-Downs, H. E. (2016). Synopsis of Sleep in Infants, Children, and Adolescents. In S. R. Pandi-Perumal (Ed.), Synopsis of Sleep Medicine. Apple Academic Press.

Schild, H. H. (1999). MRI made easy. Wayne, NJ: Berlex Laboratories Inc.

Semrud-Clikeman, M., Walkowiak, J., Wilkinson, A., \& Butcher, B. (2010). Executive functioning in children with asperger syndrome, ADHD-combined type, ADHD-predominately inattentive type, and controls. Journal of Autism and Developmental Disorders, 40(8), 1017-1027. http://doi.org/10.1007/s10803-010-0951-9

Shashi, V., Francis, A., Hooper, S. R., Kranz, P. G., Zapadka, M., Schoch, K., ... Keshavan, M. S. (2012). Increased corpus callosum volume in children with chromosome $22 q 11.2$ deletion syndrome is associated with neurocognitive deficits and genetic polymorphisms. European Journal of Human Genetics, 20(10), 1051-1057. http://doi.org/10.1038/ejhg.2012.138

Shaw, P. J., Tononi, G., Greenspan, R. J., \& Robinson, D. F. (2002). Stress response genes protect against lethal effects of sleep deprivation in Drosophila. Nature, 417(6886), 287-91. http://doi.org/10.1038/417287a

Short, M. a, \& Banks, S. (2014). The Functional Impact of Sleep Deprivation, Sleep Restriction, and Sleep 
Fragmentation. In M. T. Bianchi (Ed.), Sleep Deprivation and Disease- Effects on the Body, Brain, and Behavior (pp. 13-26). New York, NY: Springer. http://doi.org/10.1007/978-1-4614-9087-6

Shukla, D. K., Keehn, B., \& Muller, R.-A. (2011). Tract-specific analysis of diffusion tensor imaging show widespread white matter compromise in autism spectrum disorder. Journal of Child Psychology and Psychiatry, 52(3), 286-295. http://doi.org/10.1007/s12671-013-0269-8.Moving

Silk, T. J., Vance, A., Rinehart, N., Bradshaw, J. L., \& Cunnington, R. (2009). White-matter abnormalities in attention deficit hyperactivity disorder: A diffusion tensor imaging study. Human Brain Mapping, 30(9), 2757-2765. http://doi.org/10.1002/hbm.20703

Simon, G. E., \& Vonkorff, M. (1997). Prevalence, burden, and treatment of insomnia in primary care. American Journal of Psychiatry, 154(10), 1417-1423. http://doi.org/10.1176/ajp.154.10.1417

Smith, C. T., Nixon, M. R., \& Nader, R. S. (2004). Posttraining increases in REM sleep intensity implicate REM sleep in memory processing and provide a biological marker of learning potential. Learning \& Memory, 11(6), 714-719. http://doi.org/10.1101/Im.74904

Soares, J. M., Marques, P., Alves, V., \& Sousa, N. (2013). A hitchhiker's guide to diffusion tensor imaging. Frontiers in Neuroscience, 7(31), 1-14. http://doi.org/10.3389/fnins.2013.00031

Sotelo, C. E. (2007). Restless Legs Syndrome. In N. Butkov \& T. Lee-Chiong (Eds.), Fundamentals of Sleep Technology (pp. 154-158). Philadelphia, PA: Lippincott Willaims \& Wilkins.

Stores, G. (2008). Rapid eye movement sleep behaviour disorder in children and adolescents. Developmental Medicine and Child Neurology, 50(10), 728-732. http://doi.org/10.1111/j.14698749.2008.03071.x

Sullivan, E. V., Rosenbloom, M. J., Desmond, J. E., \& Pfefferbaum, A. (2001). Sex differences in corpus callosum size: Relationship to age and intracranial size. Neurobiology of Aging, 22(4), 603-611. http://doi.org/10.1016/S0197-4580(01)00232-9

Takahashi, Y., Kipnis, D. M., \& Daughaday, W. H. (1968). Growth hormone secretion during sleep. The Journal of Clinical Investigation, 47(9), 2079-2090. http://doi.org/10.1172/JCI105893

Tan, H.-L., Gozal, D., Ramirez, H. M., Bandla, H. P. R., \& Kheirandish-Gozal, L. (2014). Overnight polysomnography versus respiratory polygraphy in the diagnosis of pediatric obstructive sleep apnea. Sleep, 37(2), 255-60. http://doi.org/10.5665/sleep.3392

Tanaka-Arakawa, M. M., Matsui, M., Tanaka, C., Uematsu, A., Uda, S., Miura, K., ... Noguchi, K. (2015). Developmental changes in the corpus callosum from infancy to early adulthood: A structural magnetic resonance imaging study. PLOS ONE, 10(3), 1-13.

http://doi.org/10.1371/journal.pone.0118760

Taub, G. E., \& McGrew, K. S. (2004). A Confirmatory Factor Analysis of Cattell-Horn-Carroll Theory and Cross-Age Invariance of the Woodcock-Johnson Tests of Cognitive Abilities III. School Psychology Quarterly, 19(1), 72-87. http://doi.org/10.1521/scpq.19.1.72.29409

Toplak, M. E., West, R. F., \& Stanovich, K. E. (2013). Practitioner Review: Do performance-based measures and ratings of executive function assess the same construct? The Journal of Child Psychology and Psychiatry, 54(2), 131-143. http://doi.org/10.1111/jcpp.12001 
Trapp, B. D., \& Nave, K.-A. (2008). Multiple sclerosis: an immune or neurodegenerative disorder? Annual Review of Neuroscience, 31, 247-69. http://doi.org/10.1146/annurev.neuro.30.051606.094313

Tsai, F.-J., Chiang, H.-L., Lee, C.-M., Gau, S. S.-F., Lee, W.-T., Fan, P.-C., ... Chiu, Y.-N. (2012). Sleep problems in children with autism, attention-deficit hyperactivity disorder, and epilepsy. Research in Autism Spectrum Disorders, 6(7), 413-421. http://doi.org/10.1016/j.rasd.2011.07.002

Vestergaard, M., Madsen, K. S., Baare, W. F. C., Skimminge, A., Ejersbo, L. R., Ramsoy, T. Z., ... Jernigan, T. I. (2011). White matter microstructure in superior longitudinal fasciculus associated with spatial working memory performance in children. Journal of Cognitive Neuroscience, 23(9), 2135-2146. http://doi.org/10.1162/jocn

Weaver, T. E., Mancini, C., Maislin, G., Cater, J., Staley, B., Landis, J. R., ... Kuna, S. T. (2012). Continuous positive airway pressure treatment of sleepy patients with milder obstructive sleep apnea: Resultsof the CPAP apnea trial north american program (CATNAP) randomized clinical trial. American Journal of Respiratory and Critical Care Medicine, 186(7), 677-683. http://doi.org/10.1164/rccm.201202-02000C

Wolff, J. J., Gu, H., Gerig, G., Elison, J. T., Styner, M., Gouttard, S., ... Piven, J. (2012). Differences in White Matter Fiber Tract Development Present From 6 to 24 Months in Infants With Autism. Am J Psychiatry, 169(6), 589-600.

Woodcock, R. W., McGrew, K. S., \& Mather, N. (2001). Woodcock-Johnson III. Itasca, IL.

Woodward, L. J., Clark, C. A. C., Bora, S., \& Inder, T. E. (2012). Neonatal White Matter Abnormalities an Important Predictor of Neurocognitive Outcome for Very Preterm Children. PLOS ONE, 7(12), e51879. http://doi.org/10.1371/journal.pone.0051879

Wozniak, J. R., \& Lim, K. O. (2006). Advances in white matter imaging: A review of in vivo magnetic resonance methodologies and their applicability to the study of development and aging. Neuroscience and Biobehavioral Reviews, 30(6), 762-774. http://doi.org/10.1016/j.neubiorev.2006.06.003

Yendiki, A., Koldewyn, K., Kakunoori, S., Kanwisher, N., \& Fischl, B. (2013). Spurious group differences due to head motion in a diffusion MRI study. Neurolmage, 88, 79-90. http://doi.org/10.1016/j.neuroimage.2013.11.027

Yendiki, A., Panneck, P., Srinivasan, P., Stevens, A., Zollei, L., Augustinack, J., ... Fischl, B. (2011). Automated probabilistic reconstruction of white-matter pathways in health and disease using an atlas of the underlying anatomy. Frontiers in Neuroinformatics, 5(23). http://doi.org/doi: 10.3389/fninf.2011.00023 


\section{Appendix A}

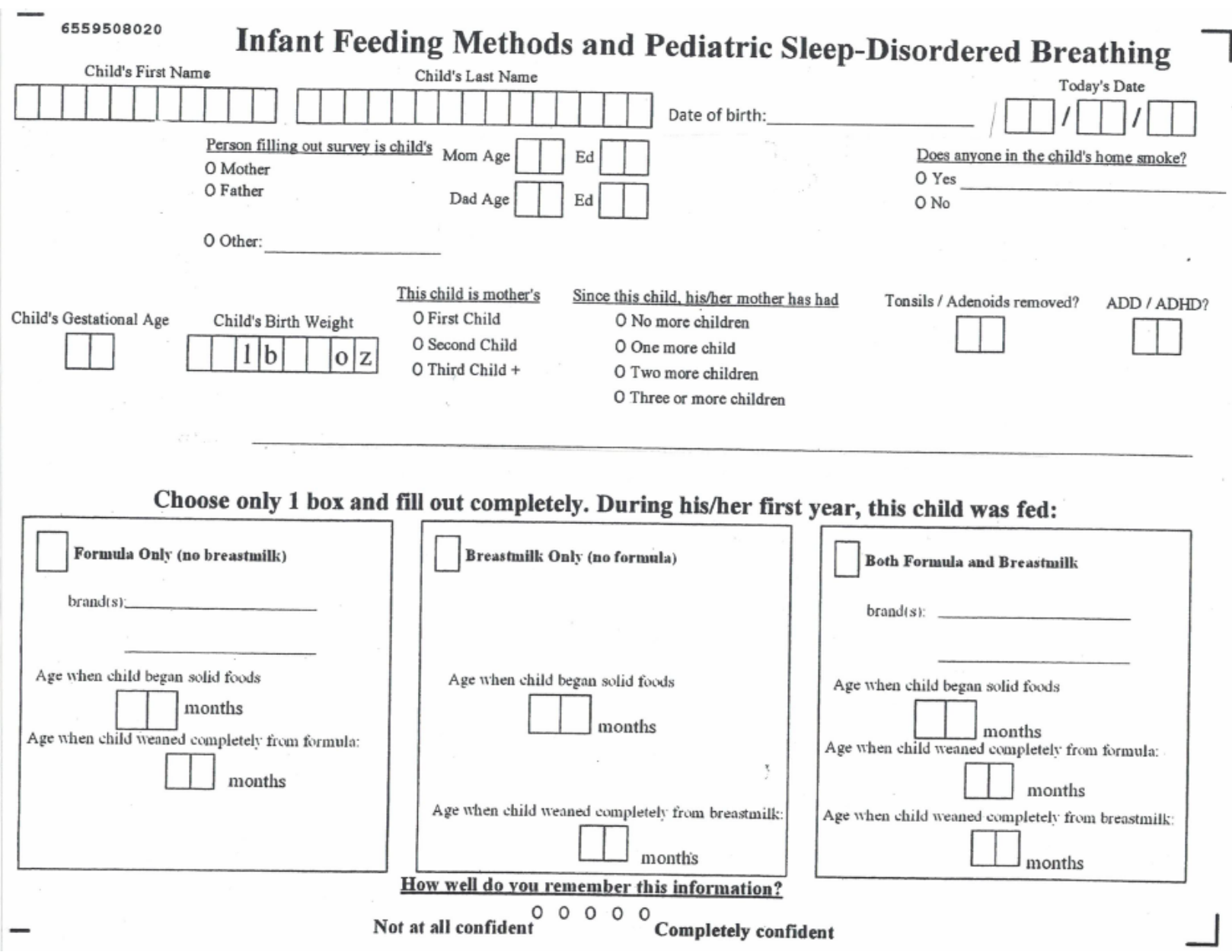

Basic demographics and infant feeding methods form. This information was acquired from the participant immediately after consent. Generally, an investigator would help the participant's parents through the demographic questions and answer any questions. 
Appendix B

Participant Number:

This information will help us make sure your child has the best experience possible in our research study. Please listen to each item and consider how well it applies to your child recently (in the past 6 months):

$\mathbf{N}$ if your child has never behaved this way

$\mathbf{S}$ if your child has sometimes behaved this way

$\mathbf{O}$ if your child has often behaved this way

A if your child has almost always behaved this way

There are no right or wrong answers. It is fine to ask me to repeat these options any time. My child...

\begin{tabular}{|l|l|l|l|l|}
\hline $\begin{array}{l}\text { 1. Has trouble concentrating on one thing, is easily distracted, and } \\
\text { seems to daydream. (add: He/she moves from activity to activity) }\end{array}$ & $\mathbf{N}$ & $\mathbf{S}$ & $\mathbf{~}$ & $\mathbf{A}$ \\
\hline $\begin{array}{l}\text { 2. Can be disobedient or defiant with adults, becomes argumentative, } \\
\text { and breaks rules. }\end{array}$ & $\mathbf{N}$ & $\mathbf{S}$ & $\mathbf{~}$ & $\mathbf{A}$ \\
\hline $\begin{array}{l}\text { 3. Doesn't follow directions well (e.g., can't wait their turn, can't sit still, } \\
\text { talks too much, etc.). }\end{array}$ & $\mathbf{N}$ & $\mathbf{S}$ & $\mathbf{~}$ & $\mathbf{A}$ \\
\hline $\begin{array}{l}\text { 4. Acts impulsively or tries to show off (e.g., is a class clown) and needs a } \\
\text { lot of attention. }\end{array}$ & $\mathbf{N}$ & $\mathbf{S}$ & $\mathbf{O}$ & $\mathbf{A}$ \\
\hline $\begin{array}{l}\text { 5. Won't leave my side (add: He/she clings to me) or worries about } \\
\text { his/her parents/caregivers. }\end{array}$ & $\mathbf{N}$ & $\mathbf{S}$ & $\mathbf{O}$ & $\mathbf{A}$ \\
\hline 6. Is afraid of tight spaces and/or loud noises. & $\mathbf{N}$ & $\mathbf{S}$ & $\mathbf{O}$ & $\mathbf{A}$ \\
\hline $\begin{array}{l}\text { 7. Whines and is constantly seeking out help (e.g., with homework, } \\
\text { chores, etc.). }\end{array}$ & $\mathbf{N}$ & $\mathbf{S}$ & $\mathbf{O}$ & $\mathbf{A}$ \\
\hline 8. Is accident prone and/or has problems controlling his/her body. & $\mathbf{N}$ & $\mathbf{S}$ & $\mathbf{O}$ & $\mathbf{A}$ \\
\hline $\begin{array}{l}\text { 9. Has temper tantrums when he/she doesn't get his/her way } \\
\text { immediately and has trouble calming down afterwards. }\end{array}$ & $\mathbf{N}$ & $\mathbf{S}$ & $\mathbf{O}$ & $\mathbf{A}$ \\
\hline $\begin{array}{l}\text { 10. Gets unduly nervous during tests or when doing homework (add: } \\
\text { concerned about how others may evaluate his/her work) }\end{array}$ & $\mathbf{N}$ & $\mathbf{S}$ & $\mathbf{O}$ & $\mathbf{A}$ \\
\hline
\end{tabular}

Notes:

Behavioral screening questionnaire used for participants. Often, this was administered even before consent in order to be best prepared for behavioral issues, although this varied. This information was not used in data analysis. 
Appendix C

\section{MAGNETIC RESONANCE IMAGING (MRI) \\ SAFETY SURVEY: \\ SESSION INFORMATION -- CHILDREN \\ This information is strictly confidential. Please PRINT LEGIBLY.}

Child's Name:

Today's Date:

ID

code:

Current weight: (pounds):

Current height (feet/inches):

Date of Birth:

Grade:

Sex:

What is the child's primary spoken language?

Of what ethnic/racial group or groups do you consider your child a member? Please indicate any that apply:

Asian African American

Hispanic

Native American

White

Other

Parent/Legal Guardian Name:

Current Occupation:

Annual Household income range:

Current Address:

email:

Current Home Phone:

Work/Cell Phoner:

If you think you might change address or telephone numbers in the next few years, may we have a more permanent address and phone through which we could locate you? Check here if not applicable: N.A.

Permanent Address:

Permanent Phone:

1. Please list all medications your child is taking, both prescription and over-the-counter (pain relievers, etc): 
2. Please describe any medical conditions your child has been diagnosed with or treated for:

3. For the following questions, please circle $\mathrm{Y}$ or $\mathbf{N}$, and fill in blanks that apply to your child:

Y N Do you considered him/her to be claustrophobic?

Y N Does he/she have a learning difficulty or has he/she been enrolled in special education classes?

Y N Has he/she been diagnosed with or have you thought he/she might have an attention problem?

Y N Has he/she been diagnosed with a hearing disorder?

If yes, please explain

Y N Has he/she ever had a head injury with loss of consciousness?

If yes, please explain and include length of time

Y N Has he/she ever had seizures, fainting spells, or migraines?

If yes, please explain

Y N Has he/she ever been evaluated for a neurological disorder?

If yes, please explain

Y N Has he/she ever been evaluated for a psychological disorder?

If yes, please explain

Y N Has he/she ever been treated for (or have you thought he/she needed treatment) for alcohol or drug abuse?

Y N Has he/she had any major surgeries or received long-term treatment for any illness?

If yes, please explain

Y N Has he/she ever received medical care at Hospitals or Clinics at West Virginia University or Mon General?

Y N Does he/she drink caffeine? If yes, how many drinks (cups of soda or hot chocolate) per day on average?

Y N Does he/she have any metal in their body (pacemaker, plates, clips, pins, rods, joints, pellets, etc.)?

Y N Does he/she wear dental fixtures, braces, or a non-removable orthodontic retainer?

$\mathrm{Y} N$ Does he/she have any hearing loss that you are aware of?

Y N Is he/she blind or visually impaired?

Y N Does he/she wear glasses or contacts? Please check one or both:_glasses contacts 
If yes, is he/she: _ nearsighted _ farsighted Vision correction, if known: Y N Do he/she have any visual problem not correctable by lenses, such as color blindness or astigmatism?

MRI safety screening form used to protect participants. The primary purpose of this form is to ensure that all participants are entirely safe for MRI scanning procedures. 
Appendix D

\begin{tabular}{|c|c|c|c|c|c|c|}
\hline Participant & Analysis & Reason & Analyzed T2Scan & Voxel dimensions & TR & $\mathrm{TE}$ \\
\hline 125 & No & OOV & & & & \\
\hline 146 & No & OOY & & & & \\
\hline 173 & No & Blurred & & & & \\
\hline 219 & No & OOV & & & & \\
\hline 222 & No & Blurred & & & & \\
\hline 235 & No & DNP & & & & \\
\hline 245 & No & OOY & & & & \\
\hline 313 & Yes & & Slow Saggital & $1.0 \times 1.0 \times 2.0$ & 10000 & 70.0 \\
\hline 328 & Yes & & Slow Saggital & $1.0 \times 1.0 \times 2.0$ & 10500 & 70.0 \\
\hline 369 & No & Blurred & & & & \\
\hline 395 & No & Blurred/oOy & & & & \\
\hline 418 & Yes & & Slow Saggital & $1.0 \times 1.0 \times 2.0$ & 10500 & 70.0 \\
\hline 429 & No & OOV & & & & \\
\hline 444 & No & DNP & & & & \\
\hline 564 & No & Technical Error & & & & \\
\hline 568 & Yes & & Slow Saggital & $1.0 \times 1.0 \times 2.0$ & 9180 & 70.0 \\
\hline 571 & Yes & & Slow Saggital & $1.0 \times 1.0 \times 2.0$ & 9180 & 70.0 \\
\hline 581 & No & DNP & & & & \\
\hline 627 & Yes & & Slow Saggital & $1.0 \times 1.0 \times 2.0$ & 10500 & 70.0 \\
\hline 686 & No & OOV & & & & \\
\hline 689 & No & DNP & & & & \\
\hline 775 & No & OOV & & & & \\
\hline 777 & Yes & & Slow Saggital & $1.0 \times 1.0 \times 2.0$ & 10500 & 70.0 \\
\hline 811 & Yes & & Slow Saggital & $1.0 \times 1.0 \times 2.0$ & 9180 & 70.0 \\
\hline 856 & No & OOY & & & & \\
\hline 885 & Yes & & Slow Saggital & $1.0 \times 1.0 \times 2.0$ & 10500 & 70.0 \\
\hline 944 & Yes & & Slow Saggital & $1.0 \times 1.0 \times 2.0$ & 10500 & 70.0 \\
\hline 946 & No & DNP & & & & \\
\hline 993 & Yes & & Slow Saggital & $1.0 \times 1.0 \times 2.0$ & 9180 & 70.0 \\
\hline
\end{tabular}

Summary of participant information in aim 1 for the T2-weighted MRI scans. Reasons for exclusion included did not participate (DNP), CC out of the field of view (OOV), or significant blurring such that an accurate CC ROI was impossible. One participant was excluded due to a technical error. Therefore, a total of 11 T2-weighted images were used in the manual CC measurements. T1-weighted MPRAGE scans were used in automated CC measurement, as Freesurfer does not analyze T2-weighted scans. Voxel dimension are reported in cubic millimeters, while repetition time (TR) and echo time (TE) are reported in milliseconds. 
Appendix $\mathrm{E}$

\begin{tabular}{|c|c|c|c|c|c|c|c|}
\hline Participant & Plane of Acq & Voxel Dimensions & TR & TE & Flip & Matrix & Fov \\
\hline 146 & Axial & $0.625 \times 0.625 \times 1.5$ & 2300 & 3.81 & 9 & $312 \times 384$ & $195 \times 240$ \\
\hline 245 & Axial & $0.625 \times 0.625 \times 1.5$ & 2300 & 3.77 & 9 & $312 \times 384$ & $195 \times 240$ \\
\hline 429 & Axial & $0.625 \times 0.625 \times 1.5$ & 2300 & 3.81 & 9 & $312 \times 384$ & $195 \times 240$ \\
\hline 571 & Saggital & $0.625 \times 0.625 \times 1.5$ & 2300 & 3.77 & 9 & $312 \times 384195 \times 240$ \\
\hline 686 & Axial & $0.625 \times 0.625 \times 1.2$ & 2300 & 3.81 & 9 & $312 \times 384195 \times 240$ \\
\hline 775 & Axial & $0.625 \times 0.625 \times 1.5$ & 2300 & 3.81 & 9 & $312 \times 384195 \times 240$ \\
\hline 777 & Axial & $0.625 \times 0.625 \times 1.5$ & 2300 & 3.77 & 9 & $312 \times 384195 \times 240$ \\
\hline 856 & Axial & $0.625 \times 0.625 \times 1.5$ & 2300 & 3.81 & 9 & $312 \times 384195 \times 240$ \\
\hline 885 & Saggital & $0.625 \times 0.625 \times 1.5$ & 2300 & 3.77 & 9 & $312 \times 384195 \times 240$ \\
\hline 993 & Axial & $0.45 \times 0.45 \times 0.9$ & 1900 & 2.99 & 9 & $512 \times 448201 \times 230$ \\
\hline
\end{tabular}

Summary of participant information in aim 1 for the MPRAGE MRI scans. Participant's scans were acquired (Plane of Acq) in either the sagittal or axial plane. All ten MPRAGE scans were used in manual CC analysis; 4 were used in the automated CC method $(429,571,686,856)$. Variables included in this table are voxel size (cubic millimeters) repetition time (RT; milliseconds), echo time (TE; milliseconds), flip angle (degrees), matrix size (voxels), and field of view (square millimeters). 
Appendix $\mathrm{F}$

1. The files of interest reside on the Desktop. Path: /Desktop/Young_Paula/ChrisB/Mysteries_for_Chris/

2. Located here are all of the different participants for whom I have traced the CC volume. Please select one (list= ATR, BYT, DES, DPE, FDL, IXP, OGQ) and go into it.

3. Let's pretend you opened DES. Go ahead and type AFNI DES+acpc.BRIK into the command line. This is the underlay.

- READ - load into memory the top option (should read all under and overlays into memory)

- OVERLAY, select "Paula_DES" This is an important step. Please do NOT open COPY_DES, CCOPY_DES, or CCCOPY_DES; only Paula_DES.

- Define Datamode -> Plugins -> Draw Dataset

- Uncheck "copy dataset"

- Select choose dataset to change directly.

- Select Paula_DES +acpc as the dataset

- A mask of the upper airway is already provided for you (to blind you) the mask has a value of 4 .

4. Start on the central most mid-sagittal slice $(X=0)$.

5. Draw a complete ROI around the entire CC

a. Subdivide the CC into 3 equal-ish parts.

i. Open "Paula's table" conveniently located in the "Mysteries for Chris" folder. This provides information as to what constitutes anterior CC, central CC, and Posterior CC. Ask me if you want to know how to get these numbers yourself (it's nothing magical- really)

ii. For DES, the most posterior point in the anterior portion is 7 , so the central CC starts at 6 and ends at -14 , thus -15 is where the most anterior point of the posterior portion begins, which ends at -36 .

6. Sample: if you open the table, go look under "DES". This will give you several metrics but the most important for your purposes is last anterior Y-coordinate and first posterior Y-coordinate. The last anterior Y-coordinate (closest to the central portion of the $\mathrm{CC}$ ) and every $\mathrm{Y}$-coordinate prior encompasses the anterior portion. The first posterior $Y$-coordinate (beyond the central portion, e.g -15 for DES) and every $Y$ coordinate subsequent is the posterior portion. All points in between consist of the central portion (e.g. 6 to -14 for DES)

7. When drawing the CC, please make sure that anterior regions = 1 (Value in AFNI Editor), central $=2$, and posterior $=3$. 
8. You may use any drawing tool available, but I would suggest the points tool as it is the most accurate. You could use the filled curve if you are feeling confident but sometimes will smooth edges if you are not careful, including voxels you did not want to include. (I use the filled curve tool and outline the region just inside the borders and then go back and fill in the edges.)

9. Please go through the brain sagittally extending to $X=6$, and $X=-6$ (feel free to look at the other view for assistance). After you feel confident about those slices, please proceed to the axial view and go through the CC, making sure it looks good on that plane. Finally, check on the coronal as well. Before saving, do one last pass through the sagittal sections. (e.g. for DES, the most posterior point in the anterior portion is 7 , so the central CC starts at 6 and ends at -14, which is where the most anterior point of the posterior portion begins (-15).

10. To clear an ROI you drew, just select the Filled Curve tool in the Define Datamode and set the Value $=0$. Draw around the area you want to redo.

11. After you are finished, please be SURE to click on the "Save" button on the "Draw Dataset" GUI. You may close out AFNI at this point. Please proceed to the other participants.

12. I will type in a simple command to quantify the ROls you drew later. If you are interested, the command is 3droistats -nzvoxels -mask Paula_DES+acpc.brik 'DES+acpc.brik'. 1=anterior in cubic millimeters, $2=$ central $3=$ posterior $1+2+3=$ whole. NOTE: technically its voxel count, but since they are cubic millimeter voxels cubic millimeters becomes the unit.

Instructions for the second tracer in the manual CC ROIs. Although this process was explained verbally, the written instructions were also provided. 
Appendix G

\begin{tabular}{|r|c|c|c|c|}
\hline Participant & Scan Type & Rater 1 Measurement 1 & Rater 1 Measurement 2 & Rater 2 Measurement 1 \\
\hline 627 & T2 & 6771 & 6679 & 7121 \\
\hline 777 & MPRAGE & 8527 & 8539 & 8161 \\
\hline 885 & MPRAGE & 9090 & 9297 & 8549 \\
\hline 944 & T2 & 5964 & 5711 & 6876 \\
\hline
\end{tabular}

Table of measurements for the reliability ICC calculation for manually traced CCs. The second rater traced 4 participants for Specific Aim 1, while the primary rater traced all participants. The primary rater additionally traced 4 of the participants twice for intra-rater reliability. All measurements are expressed in cubic millimeters of the total volume of the CC traced. 
Appendix $\mathrm{H}$

1) Open terminal

2) Use command "Is" to see what is all in the directory

3) Directories can be changed with the "cd" command; to go backwards in the directories, use "cd .."

4) By default, you should start out under MRI user, using "Is" command should bring up a list of potential directories

5) Cd Desktop

6) On the desktop is a folder "ChrisB"- the data is in there, so use "cd ChrisB"

7) In this folder are the MRI subjects we have scanned, plus some other documents. Select the particular subject you need to blind me of, and change to that directory. For these instructions, I'll use QDAS 22 (Expt_LRD) as an example. So, cd Expt_LRD_QDAS22_FR08. (note; you can copy and paste this into the command line

8) As it so happens, I'll need to be blinded for BOTH the t1 MPRAGE and the T2 Axial. For now select the T2 Axial. So, cd T2_TSE_AXIEL_FS_0003

9) Inside here is the main folder with all the files. There are about 60 or so individual slice files (the .IMA files). You don't need to worry about those.

10) We look at these files using a program called AFNI. You must pull AFNI up using the command line. Also, You will need to load the file which +acpc.BRIK file (don't worry about why). So, you would put into the command line "AFNI QDAS22T2+acpc.BRIK"

11) When you launch this you should see the brain. I need you to mask regions which are not the brain. The most important part are the eyes/nose/mouth regions going up to the brain.

12) You will have to draw something called a mask. To do this, in the AFNI GUI window (pops up in the top left corner) select Define Datamode $\rightarrow$ Plugins $\rightarrow$ Draw Dataset

13) In the top window, it will ask you to copy a dataset. Select the dataset you have been viewing to make a copy of. This will rename whatever you are working on to COPY_(followed by file name).

14) Select the shape you want (I suggest open or closed curve) Pick whatever color you want. IMPORTANT!!!!! Set the value of the mask to 4 . The reason for this is that I use $1,2,3$ when I am creating my own masks.

15) You can begin drawing by holding in the wheel on the mouse and moving around

16) To undo any mistake, you may set the mask value back to zero and erase areas

17) When you are finished MAKE SURE THAT YOU SAVE the mask. This can be done by simply selecting "Save"

18) At this point you can close out AFNI 
19) Back at the command line, you will be still be in whatever subject folder you were using. You can back out of this directory by using "cd . ."

20) Select the next subject you want to blind me to and continue using the same directions

Instructions for the blinder (Caitlin M.) to draw an anatomical ROI around the upper-airway, mouth, and other non-brain regions. This ROI was intended to blind the CC raters from anatomical identifiers that could potentially un-blind them. 
Appendix I

\begin{tabular}{|c|c|c|c|c|}
\hline Participant & MPRAGE_Total & MPRAGE_Anterior & MPRAGE_Central & MPRAGE_Posterior \\
\hline 146 & 5702 & 2470 & 1266 & 1966 \\
\hline 245 & 13738 & 5211 & 3134 & 5393 \\
\hline 429 & 7019 & 2948 & 1472 & 2599 \\
\hline 571 & 7438 & 3153 & 1654 & 2631 \\
\hline 686 & 8079 & 3440 & 1637 & 3002 \\
\hline 775 & 8510 & 3383 & 1855 & 3272 \\
\hline 777 & 8527 & 4092 & 1668 & 2767 \\
\hline 856 & 6511 & 2744 & 1376 & 2391 \\
\hline 885 & 9090 & 4179 & 2071 & 2840 \\
\hline 993 & 7623 & 3360 & 1599 & 2664 \\
\hline
\end{tabular}

Manual CC measurements for participants with an MPRAGE in aim 1 expressed in cubic millimeters. Participant 245 was ultimately excluded as they were an obvious statistical outlier. 
Appendix J

\begin{tabular}{|c|c|c|c|c|}
\hline Participant & T2_Total & T2_Anterior & T2_Central & T2_Posterior \\
\hline 313 & 5558 & 2474 & 1115 & 1969 \\
\hline 328 & 5323 & 2441 & 1047 & 1835 \\
\hline 418 & 3960 & 1944 & 834 & 1182 \\
\hline 568 & 5261 & 2102 & 1124 & 2035 \\
\hline 571 & 7661 & 3273 & 1624 & 2764 \\
\hline 627 & 6771 & 2954 & 1348 & 2469 \\
\hline 777 & 7814 & 3765 & 1575 & 2474 \\
\hline 811 & 7336 & 3656 & 1564 & 2116 \\
\hline 885 & 8615 & 3999 & 1864 & 2752 \\
\hline 944 & 5964 & 2820 & 1064 & 2080 \\
\hline 993 & 6636 & 3049 & 1239 & 2348 \\
\hline
\end{tabular}

Manual CC measurements for participants with a T2-weighted scan in aim 1 expressed in cubic millimeters. 
Appendix K

1. The files are located in the Freesurfer Subjects directory. . . /Applications/Freesurfer/subjects/blinded/mri/

2. On the command line, use the following command as a template: tkmedit Blinded norm_BBB.mgz -segmentation cc_BBB.mgz \$FREESURFER_HOME/FreesurferColorLUT.txt You should only have to change the subject code name (AAA) for each subject to view them.

3. Once the command has been inserted Freesurfer will bring up the segmented Freesurfer image of the brain. The segmented version has divided the CC into 5 parts, $13 \mathrm{~mm}$ in thickness (out to $6 \mathrm{~mm}$ from midline both sides).

4. You can view the brain in 3 different planes- please begin tracing the CC on the sagittal plane (like slicing your head into 2 equal pieces down between your eyes). The segmentation can be adjusted by pushing down the wheel button on the mouse

5. Go through every slice on the sagittal, adjusting areas the automated method may have overestimated or missed (overestimation is more common)

6. After that, go through the brain axially (slicing the brain into slices going superior to inferior)

7. Then, go through coronally (slice the brain like a loaf of bread)

8. If you make an error and delete an area of the actual CC, or you want to include an area rejected by the automated method, go to tools $->$ configure segmentation brush $->C C$ segmentation (251-255). Be sure to select the appropriate region of the CC.

9. When you are done go to File -> Save Segmentation as $->$ be sure to put it in the same folder, and name it CC_caitlin_(Subject code).mgz

10. Please trace subject $A A A, B B B, C C C, E E E, J J J, R R R$

Above are the written instructions for CC adjustments in Freesurfer for the second rater. This was given in supplement to verbal instruction. The primary rater followed the same routine. 
Appendix L

\begin{tabular}{|c|c|c|c|}
\hline Participant & Rater 1 Measurement 1 & Rater 1 Measurement 2 & Rater 2 \\
\hline 119 & 6753 & 6616 & 7013 \\
\hline 333 & 5853 & 5896 & 5995 \\
\hline 483 & 6925 & 6874 & 6731 \\
\hline 571 & 7026 & 7062 & 6893 \\
\hline 775 & 7633 & 7636 & 7530 \\
\hline 888 & 7338 & 7334 & 7130 \\
\hline
\end{tabular}

Table of measurements for the reliability ICC calculation in Freesurfer. Both raters traced 2 participants for Specific Aim 1 and 4 for Specific Aim 2. The primary rater additionally traced the 6 participants twice for intra-rater reliability. All measurements are expressed in cubic millimeters of the total volume of the CC traced. 
Appendix M

\begin{tabular}{|c|c|c|c|c|c|c|}
\hline Participant & CC Total & Anterior & Anterior-Central & Central & Posterior-Central & Posterior \\
\hline 146 & 5244 & 1627 & 799 & 748 & 688 & 1382 \\
\hline 429 & 6525 & 1970 & 858 & 774 & 800 & 2123 \\
\hline 571 & 7026 & 1919 & 1102 & 880 & 920 & 2205 \\
\hline 686 & 7097 & 2122 & 1064 & 901 & 776 & 2234 \\
\hline 775 & 7633 & 2417 & 907 & 909 & 936 & 2464 \\
\hline 856 & 5948 & 1751 & 809 & 723 & 714 & 1951 \\
\hline
\end{tabular}

CC volumes in Specific Aim 1 using the semi-automated Freesurfer tracings reported in cubic millimeters. 
Appendix $\mathrm{N}$

\section{Correlations}

\begin{tabular}{|c|c|c|c|c|c|c|c|}
\hline & & eTIV & FB_LR & Intercom_LR & $\mathrm{FB}$ & Intercom & AFNI_ROI \\
\hline \multirow[t]{3}{*}{ eTIV } & Pearson Correlation & 1 & $.814^{\pi \times}$ & $.656^{\mathrm{kn}}$ & $.576^{\mathrm{Nx}}$ & .388 & $.821^{\pi \times}$ \\
\hline & Sig. (2-tailed) & & .000 & .002 & .010 & .101 & .000 \\
\hline & N & 19 & 19 & 19 & 19 & 19 & 19 \\
\hline \multirow[t]{3}{*}{ FB_LR } & Pearson Correlation & $.814^{\mathrm{kn}}$ & 1 & $.795^{\pi \times}$ & $.727^{\pi n}$ & $.478^{x}$ & $.982^{k \pi}$ \\
\hline & Sig. (2-tailed) & .000 & & .000 & .000 & .039 & .000 \\
\hline & N & 19 & 19 & 19 & 19 & 19 & 19 \\
\hline \multirow[t]{3}{*}{ Intercom_LR } & Pearson Correlation & $.656^{\text {nx }}$ & $.795^{\mathrm{xn}}$ & 1 & $.602^{\pi x}$ & $.834^{\pi n}$ & $.722^{\mathrm{xN}}$ \\
\hline & Sig. (2-tailed) & .002 & .000 & & .006 & .000 & .000 \\
\hline & N & 19 & 19 & 19 & 19 & 19 & 19 \\
\hline \multirow[t]{3}{*}{ FB } & Pearson Correlation & $.576^{\mathrm{n} \pi}$ & $.727^{x \times}$ & $.602^{x \pi}$ & 1 & $.690^{x \times}$ & $.751^{x \times}$ \\
\hline & Sig. (2-tailed) & .010 & .000 & .006 & & .001 & .000 \\
\hline & $\mathrm{N}$ & 19 & 19 & 19 & 19 & 19 & 19 \\
\hline \multirow[t]{3}{*}{ Intercom } & Pearson Correlation & .388 & $.478^{x}$ & $.834^{2 \pi}$ & $.690^{x \pi}$ & 1 & .423 \\
\hline & Sig. (2-tailed) & .101 & .039 & .000 & .001 & & .071 \\
\hline & $N$ & 19 & 19 & 19 & 19 & 19 & 19 \\
\hline \multirow[t]{3}{*}{ AFNI_ROI } & Pearson Correlation & $.821^{\pi x}$ & $.982^{2 \pi}$ & $.722^{* \pi}$ & $.751^{\pi \times}$ & .423 & 1 \\
\hline & Sig. (2-tailed) & .000 & .000 & .000 & .000 & .071 & \\
\hline & $\mathrm{N}$ & 19 & 19 & 19 & 19 & 19 & 19 \\
\hline
\end{tabular}

${ }^{*}$. Correlation is significant at the 0.01 level (2-tailed).

*. Correlation is significant at the 0.05 level (2-tailed).

Correlation table between estimated total intracranial volume (eTIV) and all other constructed variables to find an ideal replacement. Description of variables are described in Appendix 0 . Here, both the distance from the front of the brain to the back of the brain multiplied by the left-most point of the brain by the right-most (FB_LR) and a manually drawn ROI around the whole brain in AFNI (AFNI_ROI) were found to be best correlated with eTIV. Since FB_LR is a semi-automated approach, and AFNI_ROI is completely manual, FB_LR was chosen as the appropriate normalization method in the absence of eTIV. 
Appendix 0

\begin{tabular}{|c|c|c|c|c|c|c|}
\hline Participant & eTIV & FB_LR & Intercom_LR & FB & Intercom & AFNI ROI \\
\hline 119 & 1484392 & 22275 & 2754.0 & 165 & 20.4 & 17579 \\
\hline 146 & 1250795 & 19220 & 2728.0 & 155 & 22.0 & 15047 \\
\hline 149 & 1344384 & 22914 & 3108.8 & 171 & 23.2 & 17845 \\
\hline 234 & 1543346 & 23275 & 3444.7 & 175 & 25.9 & 18181 \\
\hline 316 & 1343052 & 21248 & 2969.6 & 166 & 23.2 & 16447 \\
\hline 454 & 1502784 & 23364 & 3088.8 & 177 & 23.4 & 18587 \\
\hline 483 & 1292361 & 22032 & 3060.0 & 162 & 22.5 & 17317 \\
\hline 555 & 1369194 & 22879 & 2959.2 & 167 & 21.6 & 17951 \\
\hline 654 & 1647441 & 23584 & 3108.8 & 176 & 23.2 & 18793 \\
\hline 682 & 1495606 & 22950 & 3321.0 & 170 & 24.6 & 17899 \\
\hline 694 & 1357600 & 21413 & 2806.3 & 161 & 21.1 & 17072 \\
\hline 722 & 1325296 & 21590 & 2959.1 & 170 & 23.3 & 17034 \\
\hline 749 & 1501314 & 24288 & 3339.6 & 176 & 24.2 & 18919 \\
\hline 755 & 1503749 & 22240 & 2974.6 & 160 & 21.4 & 17450 \\
\hline 775 & 1335700 & 21413 & 3045.7 & 161 & 22.9 & 16591 \\
\hline 863 & 1551008 & 23766 & 3320.5 & 164 & 22.9 & 18393 \\
\hline 888 & 1414353 & 22379 & 2988.5 & 161 & 21.5 & 17301 \\
\hline 951 & 1568762 & 23936 & 3318.4 & 176 & 24.4 & 18428 \\
\hline 972 & 1622038 & 24215 & 3436.5 & 167 & 23.7 & 18898 \\
\hline & & & & & & \\
\hline
\end{tabular}

Different normalization methods and values for 19 different MRI participants. Estimated total intercranial volume (eTIV; cubic millimeters) is the master variable with which all other experimental methods are correlated. This variable is inherently generated by Freesurfer (Fischl et al., 2004), and has been shown to be a good indicator of the intended measure. Represented variables include a manually drawn brain mask using AFNI on a single slice (AFNI ROI; square millimeters), intercommissural distance (Intercom; millimeters), distance from front of the brain to the back (FB; millimeters), intercommissural distance multiplied by the distance from the left-most point of the brain to the right-most (Intercom_LR; square millimeters), and distance from front of the brain to the back multiplied by the distance from the left-most point of the brain to the right-most (FB_LR; square millimeters). 
Appendix P

\begin{tabular}{|c|c|c|c|c|c|c|c|c|c|}
\hline Participant & WJ-III Exec & WJ-III NR & BRIEF & PPVT & DNS & CF_Time & CF_Error & PF_Time & PF_Errors \\
\hline 125 & 69 & 3 & 79 & 79 & 10 & 11.5 & 1 & 14.4 & 1 \\
\hline 146 & 63 & 16 & 87 & 27 & 8 & 53.2 & 5 & 44.8 & 0 \\
\hline 173 & 22 & 7 & 84 & 9 & 13 & 62.4 & 2 & 105.1 & 2 \\
\hline 222 & 25 & 5 & 99 & 79 & 16 & 19.0 & 0 & NA & NA \\
\hline 245 & 43 & 34 & 89 & 58 & 16 & 9.5 & 0 & 30.9 & 0 \\
\hline 313 & NA & 19 & 92 & 55 & 6 & 50.0 & 13 & NA & NA \\
\hline 328 & 25 & 12 & 97 & 75 & 14 & 42.0 & 3 & 30.0 & 0 \\
\hline 369 & 37 & 43 & 96 & 47 & 16 & 17.9 & 2 & 47.2 & 1 \\
\hline 395 & 49 & NA & 75 & 63 & 16 & 49.0 & 3 & 143.0 & 3 \\
\hline 418 & 53 & NA & 91 & 53 & 16 & 105.0 & 6 & 78.0 & 5 \\
\hline 429 & 89 & NA & 86 & 86 & 15 & 52.0 & 7 & 127.7 & 0 \\
\hline 564 & NA & 59 & 97 & 84 & 15 & 87.0 & 4 & 146.0 & 3 \\
\hline 568 & 2 & 12 & 93 & 34 & 13 & 26.0 & 1 & 54.0 & 0 \\
\hline 571 & 62 & 38 & 51 & 88 & 16 & 11.3 & 0 & 26.9 & 0 \\
\hline 627 & 70 & 19 & 42 & 73 & 16 & 27.1 & 1 & 47.9 & 0 \\
\hline 686 & 53 & 93 & 74 & 79 & 16 & 31.0 & 1 & 61.4 & 0 \\
\hline 775 & NA & 10 & 92 & 61 & 12 & 59.8 & 4 & 191.0 & 6 \\
\hline 777 & 30 & 54 & 99 & 32 & 14 & 12.6 & 0 & 20.2 & 0 \\
\hline 811 & 49 & 57 & 84 & 63 & 15 & 37.3 & 2 & 36.7 & 0 \\
\hline 856 & 53 & 11 & 94 & 77 & 13 & 17.9 & 1 & 22.5 & 0 \\
\hline 985 & 28 & 43 & 97 & 50 & 15 & 21.4 & 1 & 56.7 & 0 \\
\hline 993 & 29 & 72 & 93 & 77 & 14 & 7.5 & 3 & 59.3 & 1 \\
\hline & 57 & 47 & 87 & 77 & 13 & 74.0 & 0 & 62.0 & 0 \\
\hline
\end{tabular}

Selected neurocognitive outcomes for the participants in aim 1. Included is Woodcock-Johnson test of cognitive abilities executive cluster (version 3; WJ-III Exec) percentile, Woodcock-Johnson numbers reversed percentile specifically (WJ-III NR), Behavior Rating Inventory of Executive Function (BRIEF) percentile, Peabody Picture Vocabulary Test $4^{\text {th }}$ edition (PPVT) percentile, DayNight Stroop Test (DNS; \# correct out of 16 in experimental condition), time to complete color form test (CF_Time) and number of errors (CF_Error), time to complete progressive figures tests (PF_Time) and number of errors (PF_errors). 
Appendix Q

\begin{tabular}{|c|c|c|c|c|c|}
\hline Participant & Method & Began Solid Foods & Weaned from Breast & Weaned from Formula & Confidence \\
\hline 125 & Formula & 4 & $\mathrm{NA}$ & 12 & 5 \\
\hline 146 & Formula & 6 & $\mathrm{NA}$ & 12 & 5 \\
\hline 173 & Formula & 10 & $\mathrm{NA}$ & 12 & 5 \\
\hline 219 & Formula & 8 & $\mathrm{NA}$ & 12 & 5 \\
\hline 222 & Formula & 6 & NA & 12 & 4 \\
\hline 245 & Breast & 6 & 15 & NA & 5 \\
\hline 313 & Formula & 6 & $N A$ & 12 & 5 \\
\hline 328 & Formula & 6 & NA & 12 & 4 \\
\hline 369 & Formula & Blank & $\mathrm{NA}$ & 12 & 5 \\
\hline 395 & Both & 6 & 12 & 18 & 4 \\
\hline 418 & Both & 9 & 12 & 13 & 4 \\
\hline 429 & Breast & 10 & 13 & NA & 5 \\
\hline 564 & Formula & 5 & NA & 7 & 4 \\
\hline 568 & Both & 7 & 1 & 12 & Blank \\
\hline 571 & Both & 11 & 5 & 12 & 4 \\
\hline 627 & Both & 5 & 17 & 12 & 5 \\
\hline 686 & Both & 4 & 4 & 12 & 3 \\
\hline 775 & Both & 4 & 9 & 12 & 5 \\
\hline 777 & Breast & 10 & 10 & NA & 5 \\
\hline 811 & Both & 6 & 14 & 12 & 5 \\
\hline 856 & Both & 8 & 6 & 12 & 5 \\
\hline 885 & Formula & 9 & NA & 11 & 4 \\
\hline 944 & Formula & 6 & $\mathrm{NA}$ & 10 & 5 \\
\hline 993 & Formula & 3 & $\mathrm{NA}$ & 12 & 5 \\
\hline
\end{tabular}

Infant feeding methods used to nourish the participants when they were infants in aim 1 . The "method" refers to whether participants were exclusively breastfed, exclusively formula-fed, or a mixture of both. All values from when the child began solid foods, or was weaned from either breast or formula are reported in months. Maternal confidence was reported on a 5 point scale. 


\section{Appendix R}

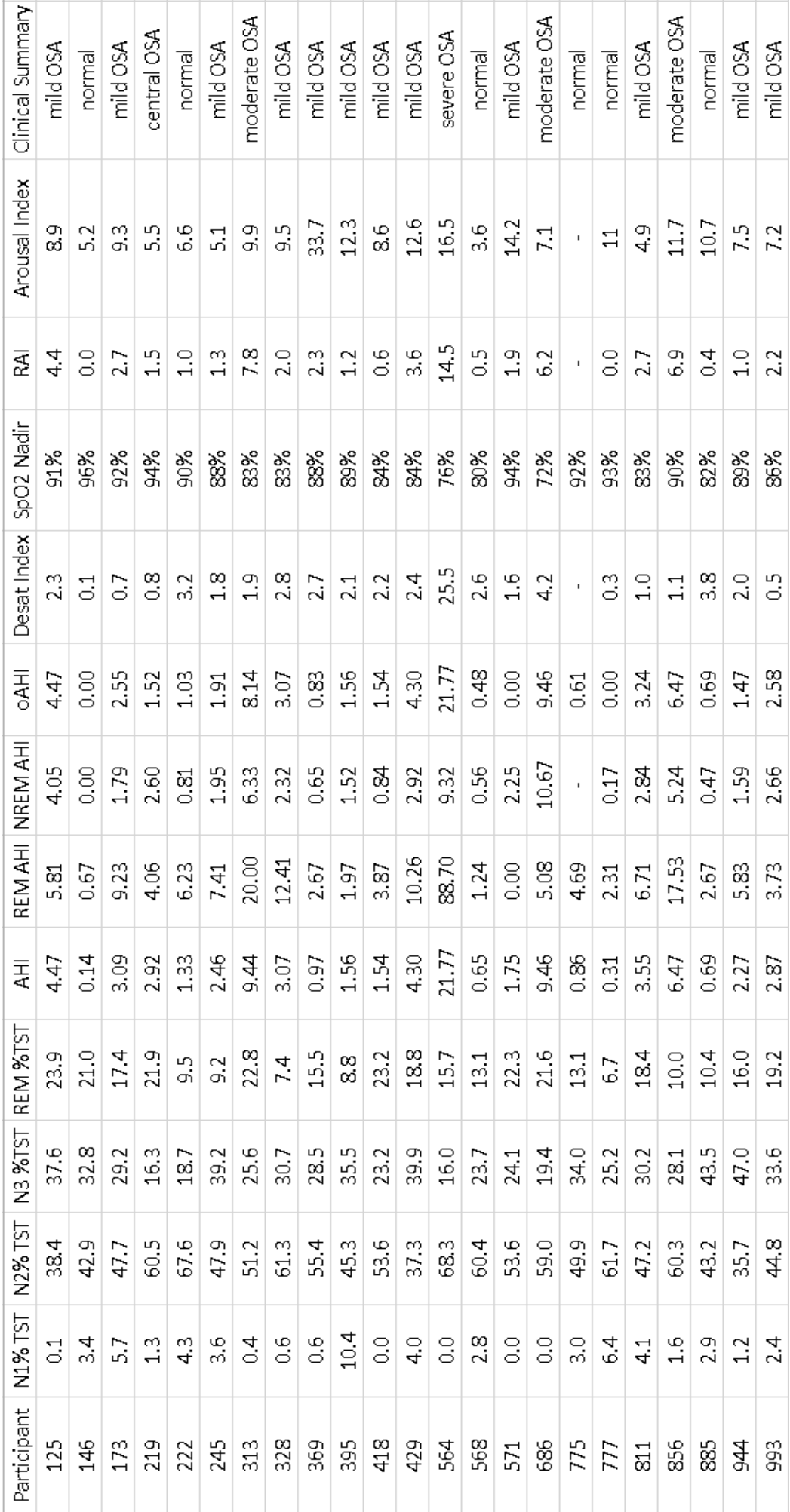

Selected sleep variables for each participant in aim 1. 


\section{Appendix S}

Initial screen: consent preamble letter.

Screening Question: Do you have a child currently between 6 months and 12 years old?

- Yes

- No

[If 'yes', participant will continue to survey. If 'no', they will be directed to the end of the survey without the option for compensation.]

If you have more than one child, please complete this information about a child living with you and who is closest to 4 years old.

1. This child's date of birth:

2. This child's gender:

3. This child's Race:

- White

- Black/African-American

- American Indian/Alaska Native

- Asian

- Native Hawaiian/other Pacific Islander

- Other (please specify)

4. Is this child of Hispanic or Latino ethnicity?

- Yes

- No

5. This child's current weight:

6. This child's current height:

7. Indicate any medical problems this child has:

8. This child's home zip code:

9. Are you this child's:

- Biological Mother

- Biological Father

- Grandmother

- Grandfather

- Adoptive Mother

- Adoptive Father

- Other 
10. This child's mother's age:

11. Mother's years of education:

- High school diploma or GED

- Some college

- College degree

- Post-graduate degree

12. Mother's total household income each year (before taxes):

13. Child's parents' marital status:

- Single

- Married/Living together

- Separated/Divorced/Widowed

14. Does mother have any other children?

- Yes

- No

15. How many other children does mother have? (if answered Yes to Q14)

16. How old is/are the other child/children? (if answered Yes to Q14)

17. During his/her first year this child was:

- Exclusively breastfed

o Age when child began solid foods:

o Age when child was completely weaned from breastmilk:

- Exclusively formula fed

o Age when child began solid foods:

o Age when child was completely weaned from formula:

- Both

o Age when child began solid foods:

o Age when child was completely weaned from breastmilk:

o Age when child was completely weaned from formula:

18. Where does this child usually sleep?

- In his/her own room alone or with siblings

- In a bed/crib in my room

- With me in my bed

- Other:

19. Does anyone living with this child smoke?

- Yes

- No

20. Is this child a restless sleeper?

- Never 
- Rarely

- Occasionally

- Frequently

- Almost Always

21. Does this child stop breathing during sleep?

- Never

- Rarely

- Occasionally

- Frequently

- Almost Always

22. Does this child struggle to breathe while asleep?

- Never

- Rarely

- Occasionally

- Frequently

- Almost Always

23. Are you ever concerned about this child's breathing during sleep?

- Never

- Rarely

- Occasionally

- Frequently

- Almost Always

24. Do you think this child's sleep is normal?

- Never

- Rarely

- Occasionally

- Frequently

- Almost Always

25. Does this child breathe through their mouth during the daytime?

- Never

- Rarely

- Occasionally

- Frequently

- Almost Always

26. How often does this child snore?

- Never

- Rarely 
- Occasionally

- Frequently

- Almost Always

27. Does this child sweat during sleep?

- Never

- Rarely

- Occasionally

- Frequently

- Almost Always

28. Does this child have noisy breathing during sleep?

- Never

- Rarely

- Occasionally

- Frequently

- Almost Always

29. Was this child born by:

- Vaginal delivery

- C-section delivery

30. When was this child's due date:

31. Did the mother experience any of the following DURING her pregnancy with this child:

- Did she snore loudly (louder than talking or loud enough to be heard through closed doors)?

- Did she often feel tired, fatigued, or sleepy during the daytime?

- Did anyone observe her stop breathing during your sleep?

- Did she have, or was she being treated for, high blood pressure?

32. If you want to be entered into a drawing for a $\$ 100$ gift card, please provide your name and mailing address [if 'yes', directed to separate survey area not linked to answers]

Survey taken by the parents of all participants as part of the screening process. Participants were only recruited if their parents indicated they did not have SDB symptoms. 
Appendix $\mathrm{T}$

\section{WestVrrginiaUniversity.}

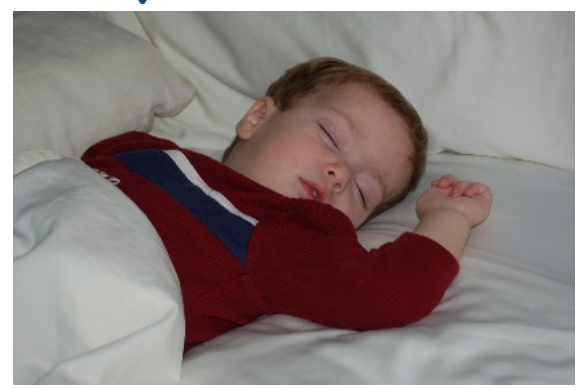

WVU Sleep Research Lab is dedicated to the prevention of child sleep \& breathing problems. If you are a parent of a child 5 months to 12 years, please complete our survey at:

https://www.surveymonkey.com/s/wvuchildsleep

$\$ 100$ gift card winners will be selected at random.

Questions? Contact: Dr. Montgomery-Downs at Hawley.Montgomery-Downs@wvu.edu.

WVU Institutional Review Board has approved this study.

Flyers used as recruitment for participants. 
Appendix U

\begin{tabular}{|c|c|c|c|c|c|c|}
\hline Participant & Survey? & Stop Breathing & Sleep Normal & Snore & Noisy Breathing & Restless Sleeper \\
\hline 863 & No & & & & & \\
\hline 234 & No & & & & & \\
\hline 149 & Yes & Never & Almost Always & Rarely & Rarely & Rarely \\
\hline 755 & Yes & Never & Almost Always & Rarely & Rarely & Never \\
\hline 951 & No & & & & & \\
\hline 682 & Yes & Never & Almost Always & Rarely & Rarely & Occasionally \\
\hline 555 & Yes & Never & Frequently & Never & Rarely & Occasionally \\
\hline 316 & Yes & Never & Almost Always & Never & Never & Rarely \\
\hline 483 & Yes & Never & Almost Always & Rarely & Rarely & Rarely \\
\hline 888 & No & & & & & \\
\hline 296 & No & & & & & \\
\hline 749 & Yes & Never & Almost Always & Rarely & Never & Never \\
\hline 972 & Yes & Never & Almost Always & Never & Never & Occasionally \\
\hline 654 & Yes & Never & Almost Always & Occasionally & Occasionally & Never \\
\hline 454 & No & & & & & \\
\hline 722 & No & & & & & \\
\hline 694 & Yes & Never & Almost Always & Rarely & Rarely & Never \\
\hline 119 & No & & & & & \\
\hline 333 & No & & & & & \\
\hline
\end{tabular}

Selected survey responses from the participants in aim 2. Although not every participant took the survey (some were screened via phone), the ones which did indicated responses that were not suggestive of SDB. 
Appendix V

\begin{tabular}{|c|c|c|c|c|}
\hline Participant & CC_Total & CC_Anterior & CC_Central & CC_Posterior \\
\hline 119 & 6549 & 2785 & 1496 & 2268 \\
\hline 149 & 6980 & 2887 & 1615 & 2478 \\
\hline 234 & 9200 & 3860 & 1959 & 3381 \\
\hline 296 & 7846 & 3526 & 1535 & 2785 \\
\hline 316 & 9861 & 4193 & 2356 & 3312 \\
\hline 333 & 6616 & 2624 & 1545 & 2447 \\
\hline 454 & 8842 & 3943 & 1947 & 2952 \\
\hline 483 & 7275 & 3148 & 1501 & 2626 \\
\hline 555 & 7729 & 3610 & 1419 & 2700 \\
\hline 654 & 7222 & 3105 & 1346 & 2771 \\
\hline 682 & 8515 & 3937 & 1788 & 2790 \\
\hline 694 & 7479 & 3133 & 1661 & 2685 \\
\hline 722 & 8001 & 3200 & 1832 & 2969 \\
\hline 749 & 7958 & 3597 & 1848 & 2513 \\
\hline 755 & 7605 & 3285 & 1625 & 2695 \\
\hline 863 & 7637 & 3404 & 1622 & 2611 \\
\hline 888 & 7609 & 3414 & 1605 & 2590 \\
\hline 951 & 7192 & 3267 & 1467 & 2458 \\
\hline 972 & 8697 & 3810 & 1910 & 2977 \\
\hline & & & & \\
\hline
\end{tabular}

Manually traced CC for each participant in aim2 (cubic millimeters). 
Appendix W
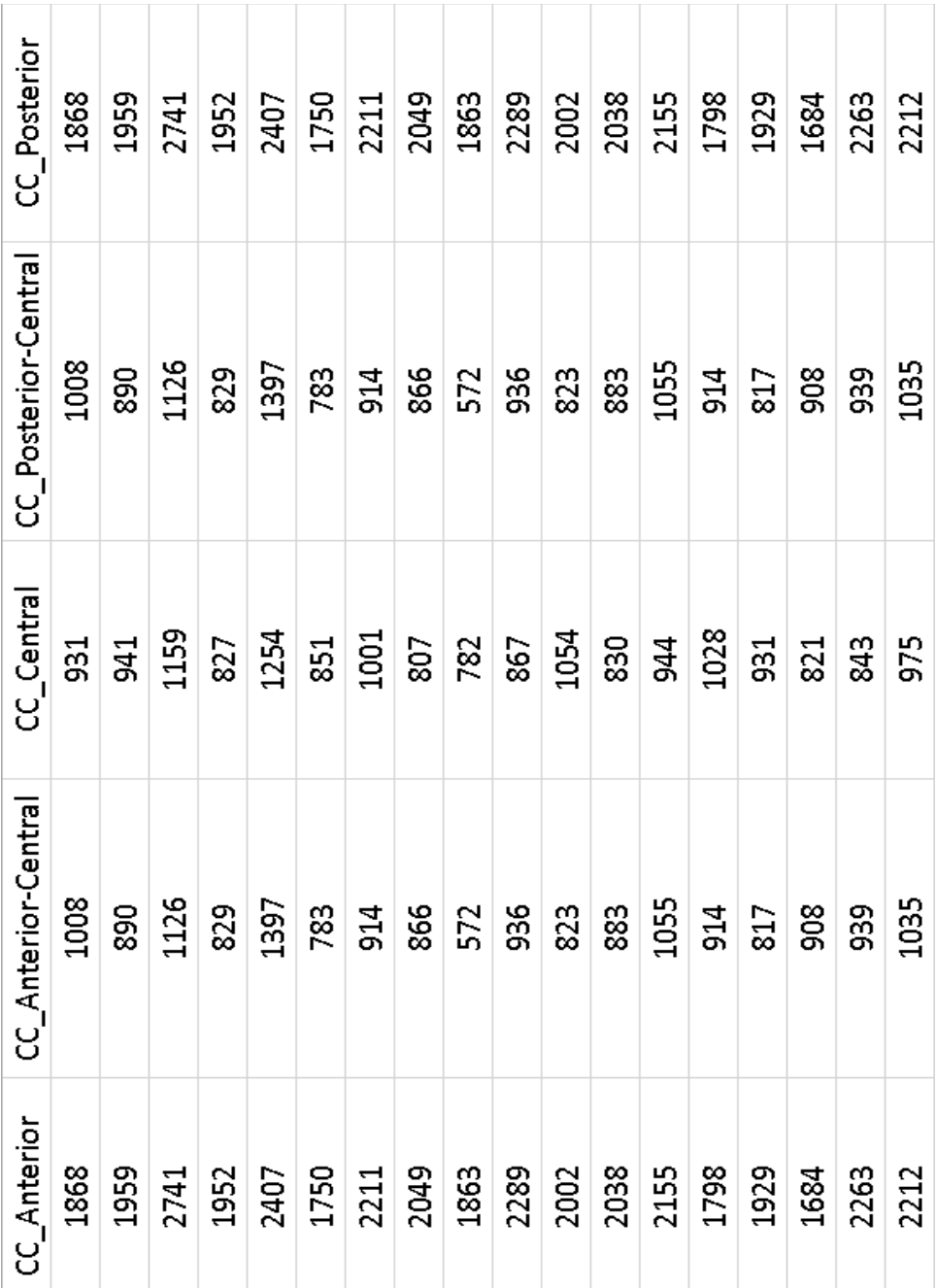

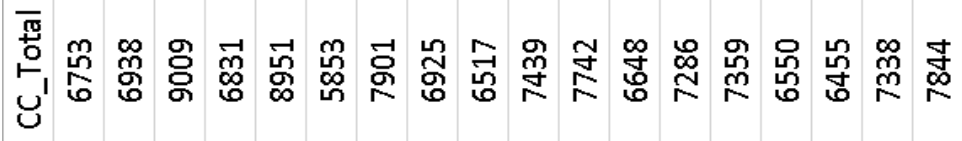

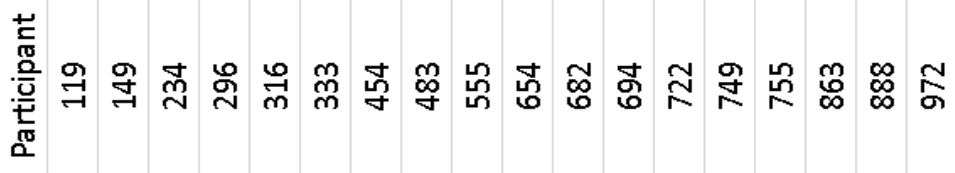

Semi-automated traced CC for each participant in aim 2 (cubic millimeters). One participant (951) was omitted due to a failure in Freesurfer to accurately trace the CC. 
Appendix X

\begin{tabular}{|c|c|c|c|c|}
\hline Participant & Scan & Rater 1 Measurement 1 & Rater 1 Measurement 2 & Rater 2 Measurement 1 \\
\hline 627 & T2 & 6771 & 6679 & 7121 \\
\hline 749 & MPRAGE & 7958 & 7766 & 7444 \\
\hline 777 & MPRAGE & 8527 & 8539 & 8161 \\
\hline 885 & MPRAGE & 9090 & 9297 & 8549 \\
\hline 944 & T2 & 5964 & 5711 & 6876 \\
\hline 972 & MPRAGE & 8697 & 8412 & 7677 \\
\hline
\end{tabular}

Reliability measurements for participants in both aims for manually traced CCs. Both aims and both scan types were sampled. 
Appendix $Y$

\begin{tabular}{|c|c|c|c|c|c|}
\hline Participant & Method & Began Solid Foods & Weaned from Breast & Weaned from Formula & Confidence \\
\hline 119 & Breast & 6 & 29 & NA & 5 \\
\hline 149 & Breast & 6 & 13 & NA & 5 \\
\hline 234 & Breast & 6 & 10 & NA & 4 \\
\hline 296 & Formula & 6 & NA & 12 & 4 \\
\hline 316 & Both & 12 & 12 & 72 & Blank \\
\hline 333 & Both & 6 & 6 & 13 & Blank \\
\hline 454 & Both & 11 & 6 & 12 & 4 \\
\hline 483 & Both & 4 & 3 & 12 & 5 \\
\hline 555 & Both & 6 & 12 & 12 & 4 \\
\hline 654 & Formula & 4 & NA & 11 & 3 \\
\hline 682 & Both & 6 & 9 & 12 & 5 \\
\hline 694 & Breast & 6 & 22 & NA & 5 \\
\hline 722 & Both & 10 & 4 & 12 & 4 \\
\hline 749 & Breast & 7 & 15 & NA & 5 \\
\hline 755 & Both & 4 & 9 & 12 & 3 \\
\hline 863 & Breast & 6 & 13 & NA & 5 \\
\hline 888 & Both & 6 & 7 & 12 & Blank \\
\hline 951 & Both & 10 & 6 & 12 & 4 \\
\hline 972 & Breast & 4 & 13 & NA & 5 \\
\hline
\end{tabular}

Infant feeding methods for participants in aim 2 reported in months. Confidence was reported on a 5 point scale. 
Appendix Z

\begin{tabular}{|c|c|c|c|c|c|}
\hline Participant & Reading & Writing & Math & Average & Work Habits \\
\hline 149 & 2.40 & 2.50 & 2.00 & 2.30 & 3.00 \\
\hline 234 & 2.00 & 2.00 & 2.25 & 2.08 & 3.00 \\
\hline 316 & 2.00 & 1.50 & 1.67 & 1.72 & 1.22 \\
\hline 454 & 2.00 & 2.00 & 2.20 & 2.07 & 2.44 \\
\hline 654 & 1.50 & 2.50 & 2.50 & 2.17 & 1.33 \\
\hline 682 & 2.60 & 2.50 & 2.67 & 2.59 & 2.00 \\
\hline 722 & 3.00 & 3.00 & 3.00 & 3.00 & 3.00 \\
\hline 755 & 2.40 & 2.50 & 2.00 & 2.30 & 3.00 \\
\hline 863 & 2.00 & 2.00 & 2.00 & 2.00 & 3.00 \\
\hline 951 & 2.25 & 2.00 & 2.25 & 2.17 & 2.67 \\
\hline
\end{tabular}

Average scores of participants based on their latest report card (from when they were scanned), normalized to the first semester of the school year. These are standardized report cards used throughout the Monongalia county schools. Report cards are divided between reading, writing, math, work habits/self-management, and sometimes science and/or social science. Since science and social science are not always included, this metric was removed. Each item can be rated needs support (NS), approaching standards (AS), meets standards (MS), or exceeds standards (ES). Each item was numerically ranked based on the teacher rating ( $N S=1, A S=2, M S=3, E S=4)$. The only exception to this is the work habits/needs support section, where each item is rated by the teacher as needs support (-), developing satisfactorily $(\checkmark)$, or demonstrates strength (+). This was also translated numerically like the other sections $(-=1, \checkmark=2,+=3)$. The reported score above represents the average of all items within the given section. The "Average" metric represents the average score between reading, writing, and math. 
Appendix AA

\begin{tabular}{|c|c|c|c|c|}
\hline Participant & Average Translation & Average Rotation & $\%$ Bad & Average Dropout Score \\
\hline 863 & 0.97 & 0.0087 & 0.00 & 1.00 \\
\hline 234 & 1.02 & 0.0111 & 0.00 & 1.00 \\
\hline 755 & 1.52 & 0.0148 & 0.04 & 1.00 \\
\hline 951 & 0.74 & 0.0050 & 0.00 & 1.18 \\
\hline 682 & 1.22 & 0.0093 & 0.04 & 1.41 \\
\hline 555 & 1.09 & 0.0113 & 0.13 & 1.00 \\
\hline 316 & 0.63 & 0.0042 & 0.00 & 1.05 \\
\hline 883 & 0.66 & 0.0064 & 0.18 & 1.08 \\
\hline 296 & 1.27 & 0.0090 & 0.08 & 1.00 \\
\hline 749 & 0.95 & 0.0049 & 0.00 & 1.00 \\
\hline 672 & 0.75 & 0.0062 & 0.00 & 1.48 \\
\hline 454 & 1.05 & 0.0086 & 0.08 & 1.23 \\
\hline 722 & 1.11 & 0.0105 & 0.58 & 1.71 \\
\hline 694 & 1.33 & 0.0110 & 0.04 & 1.00 \\
\hline
\end{tabular}

Head motion metrics calculated by TRACULA for each individual participant. Average translation is the magnitude of all volumes relative to the first volume (millimeters), average rotation is the magnitude of rotation of all volume relative to the first volume (degrees), percentage of slice with excessive signal dropout (\% Bad) is the number of slices with excess signal dropout using a proposed scoring method (Yendiki et al., 2013), and average dropout score is the magnitude of the dropout scores averaged together for the slices with excessive dropout. 
Appendix $A B$

\begin{tabular}{|c|c|c|c|}
\hline & Exclusive Breastfed $(\mathrm{n}=5)$ & Both $(\mathrm{n}=10)$ & Exclusive Formula $(\mathrm{n}=2)$ \\
\hline Avg_Translation & $1.34(0.88)$ & $1.09(0.33)$ & $1.03(0.11)$ \\
\hline Avg_Rotation & $0.01(0.01)$ & $0.009(0.004)$ & $0.008(0.004)$ \\
\hline Avg_Percent_Bad & $0.43(0.92)$ & $0.07(0.08)$ & $0.29(0.41)$ \\
\hline Avg_Dropout_Score & $1.14(0.21)$ & $1.19(0.23)$ & $1.11(0.16)$ \\
\hline
\end{tabular}

Visual inspection of means and standard deviations between feeding method groups in regards to motion measures. Neither two-way independent t-tests nor one way analysis of variance revealed any significant differences. Surprisingly, if anything, it looks like the exclusively breastfed group may have moved more than the others, but not significantly. 
Appendix AC

\section{Correlations}

\begin{tabular}{|c|c|c|c|c|c|c|}
\hline & & $\begin{array}{c}\text { Breast_Durati } \\
\text { on }\end{array}$ & Avg_Trans & Avg_Rot & Per_Bad & Avg_Drop \\
\hline \multirow[t]{3}{*}{ Breast_Duration } & Pearson Correlation & 1 & $.490^{\pi}$ & $.548^{\pi}$ & .478 & .075 \\
\hline & Sig. (2-tailed) & & .046 & .023 & .052 & .775 \\
\hline & N & 17 & 17 & 17 & 17 & 17 \\
\hline \multirow[t]{3}{*}{ Avg_Trans } & Pearson Correlation & $.490^{\pi}$ & 1 & $.970^{\mathrm{nx}}$ & $.855^{\mathrm{nn}}$ & .334 \\
\hline & Sig. (2-tailed) & .046 & & .000 & .000 & .190 \\
\hline & $N$ & 17 & 17 & 17 & 17 & 17 \\
\hline \multirow[t]{3}{*}{ Avg_Rot } & Pearson Correlation & $.548^{x}$ & $.970^{\mathrm{kx}}$ & 1 & $.877^{\mathrm{xn}}$ & .309 \\
\hline & Sig. (2-tailed) & .023 & .000 & & .000 & .227 \\
\hline & $N$ & 17 & 17 & 17 & 17 & 17 \\
\hline \multirow[t]{3}{*}{ Per_Bad } & Pearson Correlation & .478 & $.855^{\mathrm{n} x}$ & $.877^{\mathrm{nx}}$ & 1 & .130 \\
\hline & Sig. (2-tailed) & .052 & .000 & .000 & & .619 \\
\hline & $N$ & 17 & 17 & 17 & 17 & 17 \\
\hline \multirow[t]{3}{*}{ Avg_Drop } & Pearson Correlation & .075 & .334 & .309 & .130 & 1 \\
\hline & Sig. (2-tailed) & .775 & .190 & .227 & .619 & \\
\hline & $N$ & 17 & 17 & 17 & 17 & 17 \\
\hline
\end{tabular}

*. Correlation is significant at the 0.05 level (2-tailed).

**. Correlation is significant at the 0.01 level (2-tailed).

Simple correlations between breastfeeding duration and motion measures. Surprisingly, breastfeeding duration was significantly positively correlated with average translation and average rotation, suggestive that children who were breastfed longer actually moved around more in the scanner. From a theoretical perspective, this means that children with increased breastfeeding could have reduced FA scores in motion-sensitive areas. As a result of this finding, both average translation and average rotation will be included in regression models for tract analysis. 
Appendix AD

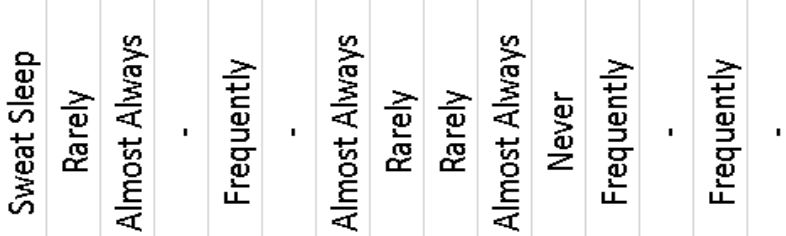

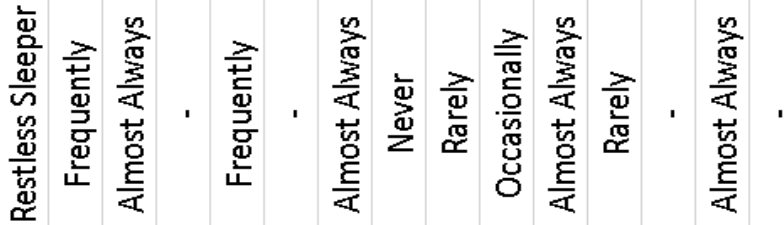

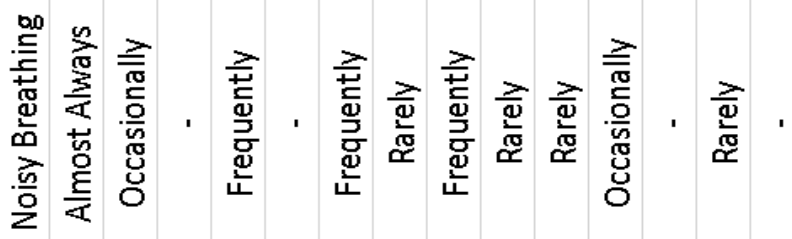

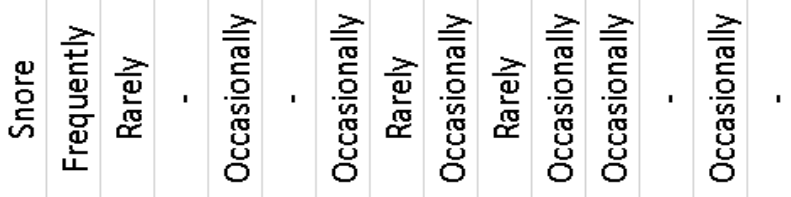

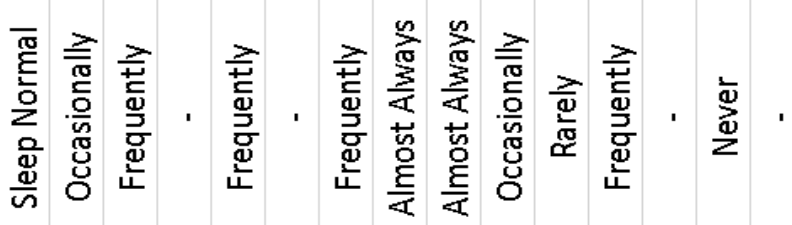

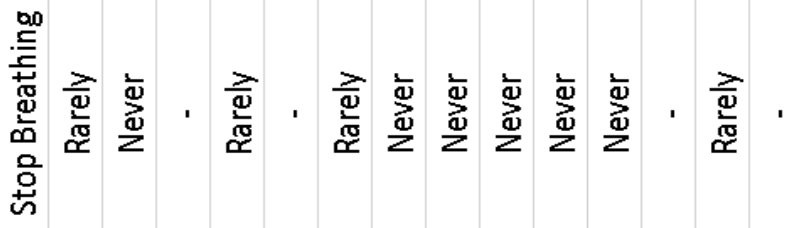

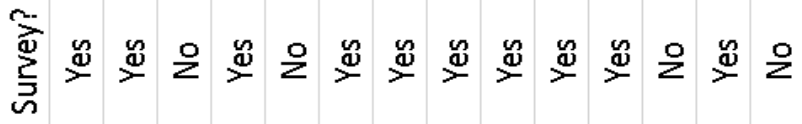

Selected survey responses from parents of participants in the polysomnography experiment in aim 3. The questions most relevant to SDB are shown above. 
Appendix AE

\section{Dear New Parent: $\quad z \quad z \quad z \quad z \quad z \quad z \quad z$ Congratulations on the birth of your child.}

Your family is invited to participate in a research study that is looking at Infants Sleep and Early Development. The study is being conducted by the Kosair Children's Hospital Research Institute, Division of Pediatric Sleep Medicine. To indicate your interest in this study please fill out the information below, fold and seal this postage-paid card and mail it to us before your baby reaches 5 months of age. You will receive more information about the benefits of the research and a brief survey about your baby's sleep when he/ she is 6 months old.

If you return this card you will also be entered in a monthly drawing for a $\$ 20$ gift card. Returning this card does not obligate you to participate in the study and if you choose not to return the survey you will not be contacted again.

If you have any questions about this research please contact Dr. Hawley Montgomery-Downs at (502) 852-2447.

We look forward to including vour family in this study.
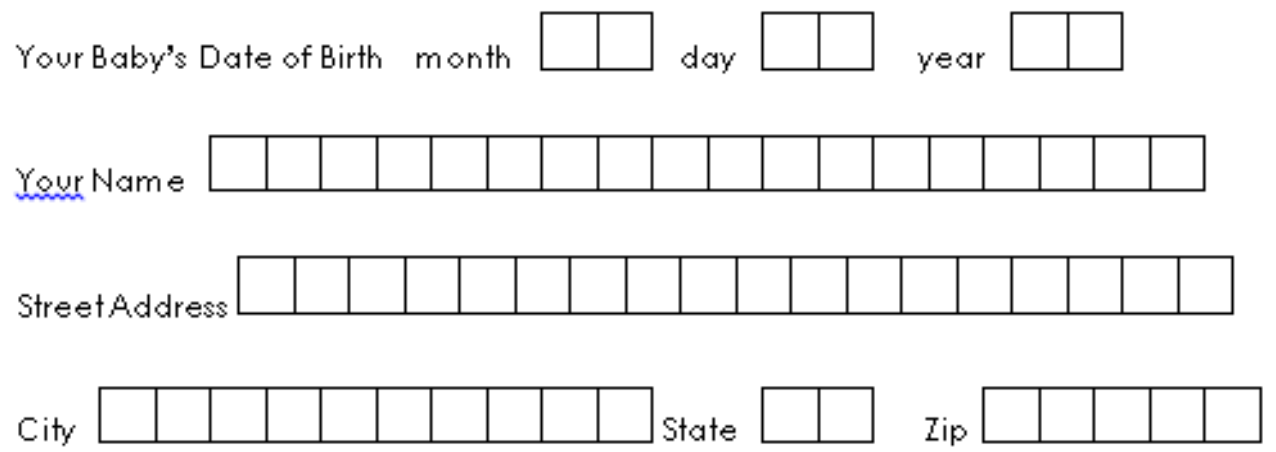


\section{Appendix AF}

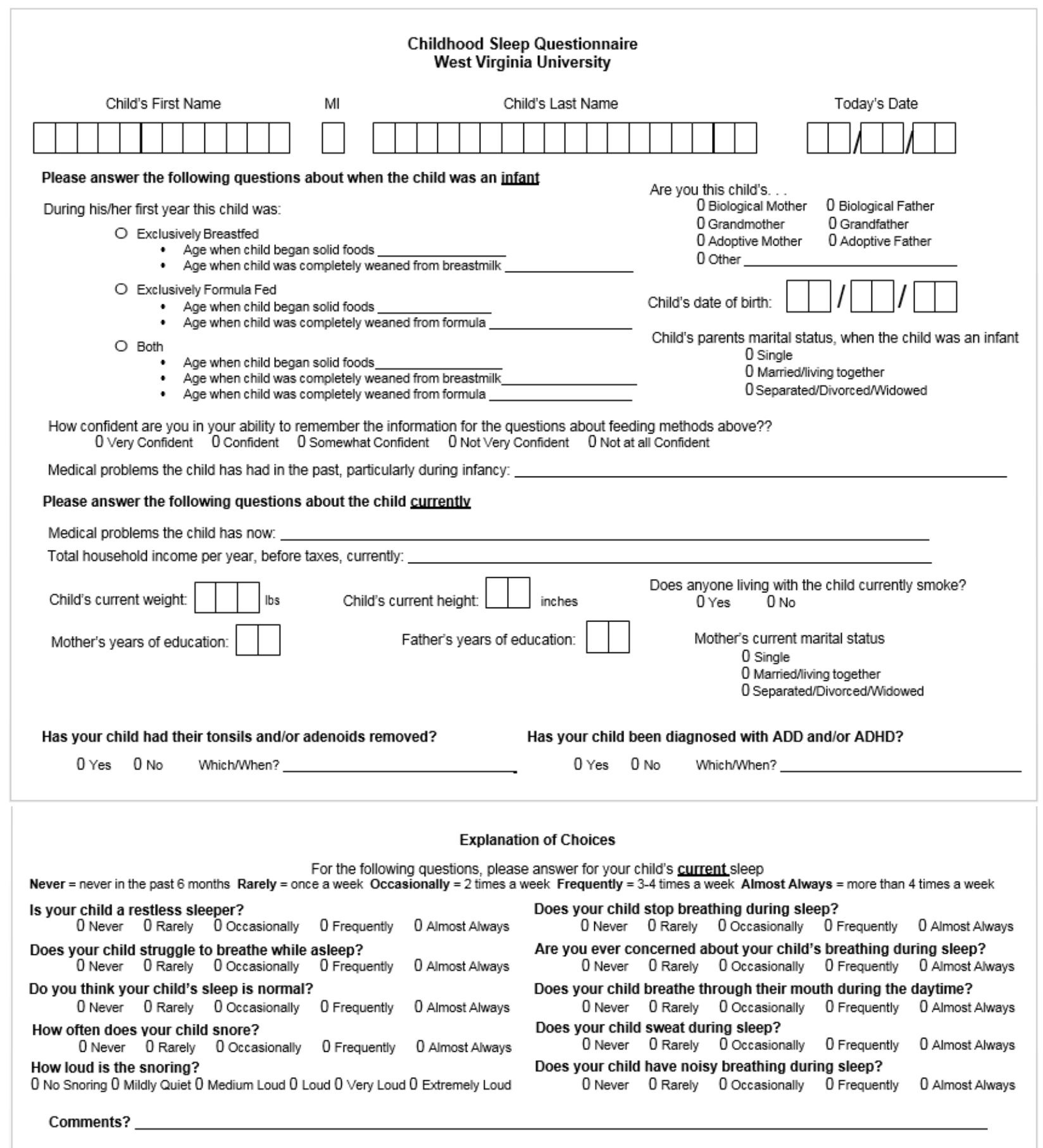

Survey for parents of participants 11 years after initial PSG. Aside from the infant feeding methods question, the sleep and breathing questions at the end are identical to what the parents answered in the original study. 
Appendix AG

\begin{tabular}{|c|c|c|c|c|c|}
\hline Participant Code & AHI & RAl & Desat_Ind & Nadir & Profile Score (Weighted Average) \\
\hline 125 & 1 & 1 & 1 & 0 & 0.83 \\
\hline 146 & 0 & 0 & 0 & 0 & 0.00 \\
\hline 173 & 1 & 1 & 0 & 0 & 0.67 \\
\hline 219 & 1 & 1 & 0 & 0 & 0.67 \\
\hline 222 & 1 & 1 & 2 & 0 & 1.00 \\
\hline 245 & 1 & 1 & 1 & 1 & 1.00 \\
\hline 313 & 2 & 2 & 1 & 2 & 1.83 \\
\hline 328 & 1 & 1 & 2 & 2 & 1.33 \\
\hline 369 & 0 & 1 & 2 & 1 & 0.83 \\
\hline 395 & 1 & 1 & 1 & 1 & 1.00 \\
\hline 418 & 1 & 0 & 1 & 2 & 0.83 \\
\hline 429 & 1 & 1 & 1 & 2 & 1.17 \\
\hline 564 & 3 & 3 & 3 & 3 & 3.00 \\
\hline 568 & 0 & 0 & 2 & 2 & 0.67 \\
\hline 571 & 1 & 1 & 1 & 0 & 0.83 \\
\hline 686 & 2 & 2 & 2 & 3 & 2.17 \\
\hline 775 & 0 & - & - & 0 & 0.00 \\
\hline 777 & 0 & 0 & 0 & 0 & 0.00 \\
\hline 811 & 1 & 1 & 1 & 2 & 1.17 \\
\hline 856 & 2 & 2 & 1 & 0 & 1.50 \\
\hline 885 & 0 & 0 & 2 & 2 & 0.67 \\
\hline 944 & 1 & 1 & 1 & 1 & 1.00 \\
\hline 993 & 1 & 1 & 0 & 1 & 0.83 \\
\hline
\end{tabular}

AHI, RAI, Desat_Ind, and SpO2 nadir were all ranked ordinally based on their composite score values as previously described (Montgomery-Downs et al., 2004). Computation of the composite score is the sum of the scores for AHI, RAI, and the average of Desat_Ind and SpO2 nadir, divided by three (AHI and RAI are weighted more heavily). 
Appendix $\mathrm{AH}$

\begin{tabular}{|c|c|c|c|}
\hline Participant & Infant Score & Current Score & Change \\
\hline 132 & 2.5 & 1.6 & 0.9 \\
\hline 136 & 1.7 & 1.2 & 0.5 \\
\hline 174 & 2.5 & 1.8 & 0.7 \\
\hline 188 & 1.4 & 1.3 & 0.1 \\
\hline 225 & 2.1 & 2.4 & -0.3 \\
\hline 258 & 1.9 & 1.3 & 0.6 \\
\hline 293 & 1.1 & 1.7 & -0.6 \\
\hline 321 & 2.3 & 2.8 & -0.5 \\
\hline 339 & 1.3 & 2.3 & -1.0 \\
\hline 357 & 1.6 & 1.5 & 0.1 \\
\hline 391 & 2.6 & 1.8 & 0.8 \\
\hline 414 & 2.1 & 1.5 & 0.6 \\
\hline 439 & 1.4 & 1.3 & 0.1 \\
\hline 484 & 1.0 & 1.2 & -0.2 \\
\hline 489 & 4.6 & 2.5 & 2.1 \\
\hline 528 & 4.2 & 1.5 & 2.7 \\
\hline 538 & 1.6 & 2.1 & -0.5 \\
\hline 542 & 1.4 & 1.4 & 0.0 \\
\hline 546 & 1.7 & 1.8 & -0.1 \\
\hline 641 & 2.0 & 2.2 & -0.2 \\
\hline 659 & 1.3 & 1.3 & 0.0 \\
\hline 667 & 1.4 & 1.6 & -0.2 \\
\hline 735 & 1.1 & 1.6 & -0.5 \\
\hline 741 & 2.8 & 2.1 & 0.7 \\
\hline 753 & 2.1 & 2.3 & -0.2 \\
\hline 798 & 2.0 & 1.5 & 0.5 \\
\hline 818 & 1.7 & 1.8 & -0.1 \\
\hline 846 & 2.4 & 1.5 & 0.9 \\
\hline 948 & 2.7 & 2.0 & 0.7 \\
\hline 963 & 1.3 & 1.5 & -0.2 \\
\hline 987 & 2.7 & 3.5 & -0.8 \\
\hline 994 & 2.1 & 1.9 & 0.2 \\
\hline
\end{tabular}

Survey questions were numerically coded ("never" $=1$, "rarely" $=2$, "occasionally"=3, "frequently"=4, "almost always"=5) except for question three which was scored in reverse. Score reported above is the average of all ten sleep questions asked. The higher the score indicates higher propensity for SDB. For each participant, a score was calculated for when the parents answered when the child was infant, their answers in the mailed survey, and the longitudinal change in the scores. The lowest aggregate score possible is one, while the highest is five. 
Appendix Al

\section{Ginger Cookies}

2 cups sugar

Makes lots

1 cup shortening

$3 / 4$ cup boiling water

1 cup baking molasses

1 egg

Add: 1 tablespoon ginger

1 tablespoon baking soda

1 teaspoon salt

Flour to stiffen (8-9 cups)

Chill in refrigerator, roll out and cut with cookie cutters. Bake at 350 degrees for 10 minutes.

\section{Goulash}

$1 \mathrm{lb}$ ground beef

15 oz tomato sauce

15 oz kidney beans

2 cups elbow macaroni

Brown ground beef, add tomato sauce, kidney beans, and cooked elbow macaroni (boiled al dente). Season to taste. Spaghetti sauce may be substituted for tomato sauce if desired. 